\title{
Superconductivity and Antiferromagnetism in the Kondo-Lattice Model
}

\author{
Dissertation
}

zur Erlangung des mathematisch-naturwissenschaftlichen Doktorgrades

„Doctor rerum naturalium”

der Georg-August-Universität Göttingen

vorgelegt von

Oliver Bodensiek

aus Gifhorn

Göttingen, 2013 
Referent: Prof. Dr. Thomas Pruschke

Korreferent: Prof. Dr. Matthias Vojta

Tag der mündlichen Prüfung: 15.08.2013 


\section{Dank}

Ich möchte mich ganz herzlich bei all denen bedanken, die mich in den letzten Jahren während der Promotion und bei der Fertigstellung dieser Arbeit unterstützt haben.

Mein besonderer Dank gilt Thomas Pruschke, der es mir ermöglicht hat, an diesem Projekt zu arbeiten. Für seine Unterstützung, seinen Rat und die große Autonomie, die ich in vielerlei Hinsicht genießen durfte, bin ich sehr dankbar. Insbesondere möchte ich auch Rok Žitko danken - für seine stetige Unterstützung und viele anregende Diskussionen. Auch für die Ermöglichung meines Forschungsaufenthalts in Ljubljana möchte ich mich an dieser Stelle noch einmal herzlich bedanken. Danke auch an Matthias Vojta für erkenntnisreiche Diskussionen und für die Übernahme des Korreferats. Die endgültige Fassung dieser Arbeit verdankt ihre Form den sorgfältigen Korrekturen und der konstruktiven Kritik von Benjamin Lenz, Alexander Tiegel, Ebad Kamil und Rok Žitko.

Meinen langzeitigen Bürokollegen Piet Dargel und Ansgar Kalz schulde ich meinen Dank für ein immer positive und auch kreative Atmosphäre im Büro, für eine gute Zeit auf Konferenzen und für die gegenseitige Unterstützung. Auch der „neuen Generation” im Büro, Alexander Tiegel, Benjamin Lenz, Fabian Biebl und Thomas Köhler vielen Dank für die Aufrechterhaltung dieser guten Atmosphäre. Der gesamten Arbeitsgruppe möchte ich für das freundliche und kollegiale Beisammensein und rege Diskussionen in Seminaren sowie bei Kaffee und Kuchen meinen Dank ausdrücken. Nicht zu vergessen ist auch die Unterstützung in vielen nicht-physikalischen Fragestellungen durch Bärbel Lütge-Hampe, Gabriele Schubert, Katrin Glormann und Heidi Afshar - vielen Dank! Jürgen Holm danke ich für die Hilfe bei vielen IT-Problemen. Auch der GWDG und dem Institut Jožef Stefan möchte ich für den IT-Support meinen Dank bekunden. Dem Institut Jožef Stefan danke ich außerdem für die Unterstützung während meines Aufenthaltes in Ljubljana. Bei der DFG bedanke ich mich für die finanzielle Förderung durch das Projekt PR293/13-1.

Ein ganz lieber Dank gilt meinen Eltern Michaela Bodensiek und Daniel Bodensiek, sowie meinem Bruder Kai Bodensiek für die immerwährende Unterstützung und Ermutigung auf jedem Weg, den ich im Leben eingeschlagen habe. Ein besonders liebes und großes Dankeschön geht an meine Freundin Maureen Raabe, für ihre uneingeschränkte Unterstützung und wundervolle gemeinsame Jahre. 



\section{Author's Note}

At the end of this thesis a complete list of all references with detailed information (all authors, title, url) is given in alphabetical order of the labels. The labels of all references are usually constructed by the first three letters of the first author and a two-digit number representing the year of publication or appearance. Deviations from this scheme are possible in the case of special characters or double names.

The references which have been published within this thesis are:

[Bod11] Bodensiek, Oliver; Zitko, Rok; Peters, Robert and Pruschke, Thomas: Low-energy properties of the Kondo lattice model. Journal of Physics: Condensed Matter (2011), 23(9): 094212, URL http: //stacks . iop.org/0953-8984/23/i=9/a=094212

[Z11] Žıtko, Rok; Bodensiek, Oliver and Pruschke, Thomas: Effects of magnetic anisotropy on the subgap excitations induced by quantum impurities in a superconducting host. Physical Review $B$ (2011), 83(5): 054512, URL http://link.aps.org/doi/10.1103/ PhysRevB.83.054512

[Bod13] Bodensiek, Oliver; Žıtko, Rok; VoJtA, Matthias; JARrell, Mark and Pruschke, Thomas: Unconventional Superconductivity from Local Spin Fluctuations in the Kondo Lattice. Physical Review Letters (2013), 110(14): 146406, URL http://link.aps.org/doi/ 10.1103/PhysRevLett.110.146406 



\section{Table of Contents}

1 Heavy Fermions and Ordered Phases 1

1.1 The Kondo Effect . . . . . . . . . . . . . . . . . . . . . . . . . . . . . . . 1

1.2 Properties of Heavy-Fermion Compounds . . . . . . . . . . . . . . . . . . . 3

$1.3 \quad$ Magnetic Order in Heavy-Fermion Materials . . . . . . . . . . . . . . . . . 5

1.4 Heavy-Fermion Superconductors: The Case of $\mathrm{CeCu}_{2} \mathrm{Si}_{2} \quad \ldots \ldots$. . . . . . 6

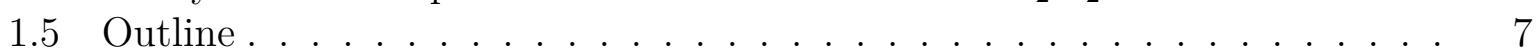

2 Computational Methods 9

2.1 Dynamical Mean-Field Theory . . . . . . . . . . . . . . . . . . . . . . . . . 10

2.1 .1 Cavity Method and Effective Action . . . . . . . . . . . . . . . . 10

2.1 .2 The Mean-Field Limit of Infinite Dimensions . . . . . . . . . . . . . 12

$2.1 .3 \quad$ Self-Consistency Equations . . . . . . . . . . . . . . . . . . . . . 14

2.1 .4 Antiferromagnetism . . . . . . . . . . . . . . . . . . . . . 16

$2.1 .5 \quad$ Superconductivity . . . . . . . . . . . . . . . . . . . . . . . . 16

2.2 Lattice Topologies . . . . . . . . . . . . . . . . . . . . . . . . . . . . . . . . 18

2.3 Quantum Impurities and their Relation to DMFT . . . . . . . . . . . . . . 20

2.4 Numerical Renormalization Group for Superconductors . . . . . . . . . . . 22

$2.4 .1 \quad$ Logarithmic Discretization . . . . . . . . . . . . . . . . . . . . 24

2.4 .2 Tridiagonalization . . . . . . . . . . . . . . . . . . . . 25

$2.4 .3 \quad$ Iterative Diagonalization . . . . . . . . . . . . . . . . . . . . 25

2.4 .4 Calculation of Static and Dynamic Properties . . . . . . . . . . . . 26

2.4 .5 Calculation of the Self-Energy . . . . . . . . . . . . . . 27

3 The Kondo-Lattice Model 29

3.1 Periodic Anderson and Kondo Lattice Model . . . . . . . . . . . . . . . . . . . 29

3.1 .1 Energy Scales and the Doniach Diagram . . . . . . . . . . . . . . 31

$3.1 .2 \quad$ Static Mean-Field Description of the KLM . . . . . . . . . . . . . . 33

3.2 Paramagnetic Properties of the Kondo-Lattice Model . . . . . . . . . . . . 38

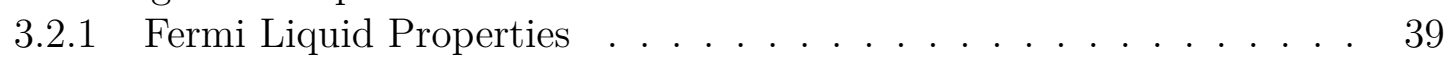

3.2 .2 Influence of Additional Interactions $\ldots \ldots \ldots$. . . . . . . . . . 42

4 The Antiferromagnetic Phase of the Kondo-Lattice Model 47

4.1 Half Filling: The Antiferromagnetic Kondo Insulator . . . . . . . . . . . . 49

4.2 Antiferromagnetism away from Half Filling . . . . . . . . . . . . . . . . . . 51

4.3 Summary . . . . . . . . . . . . . . . . . . . . . . . . . . 56 
5 Unconventional Superconductivity in the Kondo Lattice 59

5.1 Superconductivity in the KLM . . . . . . . . . . . . . . . . . 61

5.1 .1 Static Mean-Field Description of HF Superconductivity . . . . . . . 62

5.2 Superconductivity at Zero Temperature . . . . . . . . . . . . . . . 65

5.2 .1 Static Properties and Zero-Temperature Phase Diagram . . . . . . 68

5.3 Finite-Temperature Behavior . . . . . . . . . . . . . . . . 71

5.4 Pairing Mechanism . . . . . . . . . . . . . . . . . . . . . . . . . . . . 73

5.5 Eliashberg Theory for Spin-Fermion Models . . . . . . . . . . . . . . . . . 74

5.6 Summary . . . . . . . . . . . . . . . . . . . . . . . 78

$6 \quad$ Extensions of the Kondo-Lattice Model: Superconducting Properties 81

$6.1 \quad$ Stabilized Pairing in the Correlated Kondo-Lattice Model . . . . . . . . . . 82

6.2 Destabilized Pairing by Holstein Phonons . . . . . . . . . . . . . . . . . . . 86

6.3 The underscreened Kondo lattice . . . . . . . . . . . . . . . . . . . . . . . 89

7 Conclusion and Outlook 93

\begin{tabular}{ll}
\hline Appendices & 96
\end{tabular}

A Details of the DMFT Setup 99

B NRG Setup for Superconducting Leads 101

B.1 Logarithmic discretization . . . . . . . . . . . . . . . . . . . . . . . . . . . 101

B.2 Mapping to the Wilson-chain . . . . . . . . . . . . . . . . . . . . . 103

\begin{tabular}{ll}
\hline Bibliography & 107
\end{tabular} 


\section{Heavy Fermions and Ordered Phases}

Heavy-fermion (HF) materials are intermetallic compounds containing rare-earth or actinide ions with partly filled $4 f$ - and $5 f$ - orbitals, respectively. In most of these compounds the corresponding outer $f$-wavefunctions are localized inside the atomic core region due to a centrifugal barrier in their effective potential. As a result, the $f$-electrons are strongly localized and their atomic properties largely persist in the solid state since the $f$-electrons are screened by outer valence electrons. In particular, the magnetic moments originating from the $f$-shell electrons are localized; they are formed by a partial filling of the orbitals according to Hund's rule. The resulting state for Cerium is $[X e] 6 s^{2} 5 d^{1} 4 f^{1}$. In a metal containing $\mathrm{Ce}$, the outer $6 s$ - and $5 d$ - electrons contribute to the conduction band but the single $4 f$ electron remains as a stable magnetic moment in the core region of the ion. Spin-orbit coupling leads to a total angular moment $J=L-S=5 / 2$ with $L=3$ being the orbital and $S=1 / 2$ the spin angular momentum, respectively. As the Ce-ions are exposed to the crystal field of their host, this six-fold degenerate state is often split into three Kramers doublets. The low-energy physics can then typically be described by the lowest lying Kramers doublet only, provided that the relevant energy scale is lower than the crystal-field splitting. The system of Ce-ions can in this case be described as a lattice of magnetic spin-1/2 moments in a non-magnetic metallic host of conduction electrons this system is called Kondo lattice. The name stems from the Kondo effect, which describes an unusual scattering of conduction electrons by magnetic impurities. The Kondo effect, in turn, is named after Jun Kondo, who theoretically described it. Although the Kondo lattice differs in some important points from dilute alloys of magnetic impurities, it is quite instructive to consider the dilute case first.

\subsection{The Kondo Effect}

Simple metals such as copper or gold show a residual resistivity at very low temperatures $T \rightarrow 0$, which stems from electron scattering on crystal defects or neutral impurities. For low but finite temperatures, the electron-phonon scattering yields a $T^{5}$-contribution to the electrical resistivity - close to zero temperature the lattice vibrations freeze out. However, such a monotonous decrease of the resistivity to a finite value upon cooling down to $T \rightarrow 0$ is not observed in all metals. This had already been noticed by Meissner and Voigt in 1930 [Mei30a, Mei30b]. Alloying simple metals with a small amount of 
magnetic impurities, they exhibit a minimum in the resistivity at a material-specific temperature. Further decreasing the temperature, the resistivity increases again. A theoretical explanation for this unusual behavior remained a challenge to physicists for about thirty years. The first explanation was presented by Kondo via perturbation theory [Kon64. His calculations showed that the increase of the resistivity upon cooling originates from a spin-dependent scattering of conduction electrons by magnetic impurities: "spin-flip" processes, in which the spin of the impurity and the scattered electron is exchanged, give rise to a $\log (T)$-contribution to resistivity. Together with the $T^{5}$-contribution from electron-phonon scattering it qualitatively explains the existence of a minimum. However, the experimental observations do not support a logarithmically diverging resistivity for $T \rightarrow 0$ but rather a saturation. The perturbative result of Kondo thus breaks down in this regime. Later, in 1975, Wilson provided a full solution to the Kondo problem [Wil75] by means of renormalization group methods.

The spin-flip scattering at low temperatures results in a many-body effect, in which the conduction-band electrons close to the magnetic impurity screen the local moment and thereby build a many-particle singlet groundstate; only a renormalized potential scatterer in the metallic host is left. This process is called Kondo screening, which occurs below a characteristic Kondo temperature $T_{K}$. A single spin-flip is actually an exchange process on the impurity of a $d$ - or $f$ - electron and a conduction band electron with opposite spin. Hence, this interaction is often modeled by a direct antiferromagnetic exchange interaction of strength $J$. It is the consequence of a weak hybridization between localized and itinerant states. When Kondo screening is fully intact at $T \ll T_{K}$, the magnetic susceptibility in dilute magnetic alloys is similar to that of a Pauli paramagnet, $\chi \propto 1 / T_{K}$. Increasing the temperature to $T \gg T_{K}$, the system approaches a region where the impurity spin is asymptotically free and one observes a Curie-like susceptibility $\chi \propto 1 / T$. The transition regime is a continuous crossover through $T=T_{K}$, below which the local spin fluctuations appear.

The atomic states of $d$ - or $f$-orbitals together with the hybridization result in a broadened peak or resonance in the local density of states, as illustrated in (Fig. 1.1) at $U=0$. Fermi's golden rule yields a resonance width of $\Delta=\pi \rho_{0}\left(E_{f}\right) V^{2}$, where $\rho_{0}\left(E_{f}\right)$ is the density of states of the conduction electrons with an energy $E_{f}$ and $V$ denotes the average of the hybridization matrix element for transitions between conduction-band and $f$-states. Due to the strong localization of $f$-orbitals, the Coulomb interaction for two electrons in the same orbital is rather strong and splits the levels to energies $\omega=E_{f}$ and $\omega=E_{f}+U$, where only the index $f$ is used for simplicity. If the Coulomb repulsion $U$ is much larger than the broadening of the levels, the local magnetic moment is retained.

A remarkable feature in the spectral density emerges upon increasing $U$ : a third narrow and distinct resonance emerges right at the Fermi energy. It is called Abriskosov-Suhl (or Kondo) resonance and is a characteristic feature of the Kondo effect - it appears due to the strongly correlated behavior of electrons arising from scattering and it is associated with the spin 


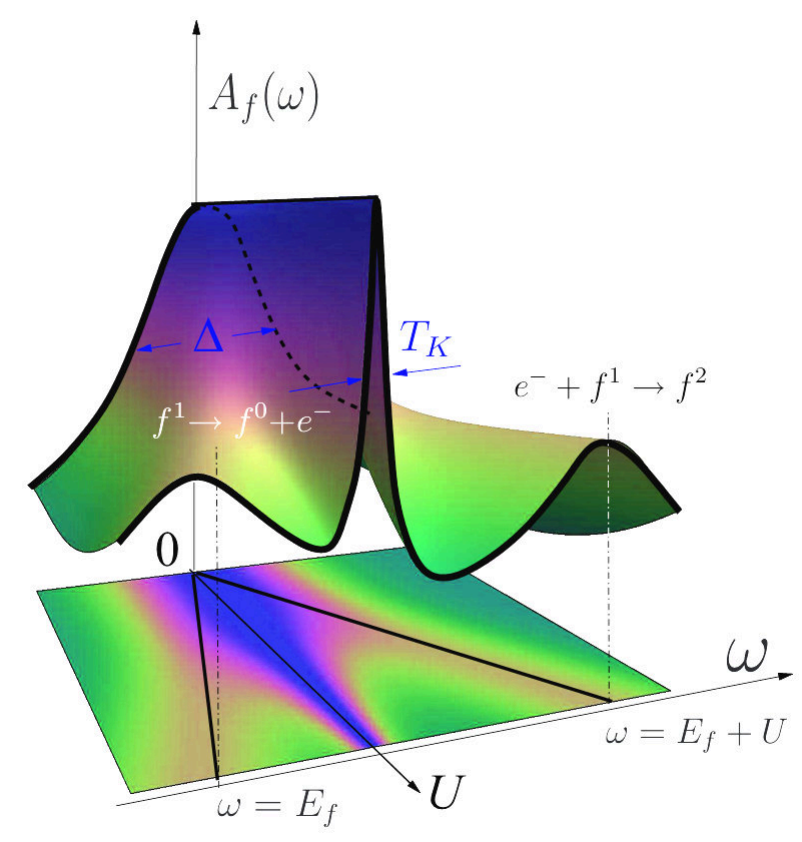

Figure 1.1: Figure adapted from Ref. [Col13]. Illustration of the formation of the Kondo resonance in the $f$-electron spectrum $A_{f}(\omega)$ with fixed $f$-occupancy. The lower part of the figure is the corresponding density plot of $A_{f}(\omega)$

fluctuations of the local moment. The renormalized width of the Abrikosov-Suhl resonance is a comparatively small energy scale of order $T_{K}$. Nevertheless, it is the origin of anomalies in the resistivity, specific heat or magnetic susceptibility, which are experimentally observed at low temperatures. In this regime, such transport and thermodynamic properties are mainly determined by electrons with an energy close to the Fermi energy.

\subsection{Properties of Heavy-Fermion Compounds}

The Kondo effect is the driving force behind the HF behavior described in the following. While thermodynamic properties of HF systems can mostly be understood in terms of single-impurity behavior, transport properties and ordered phases can only be explained by the emerging coherence effects at low temperatures. As in the dilute case, the magnetic susceptibility shows a Curie-Weiss behavior at high temperatures and a quenching of local moments at low temperatures. HF systems are considered as heavy Fermi liquids with a strongly enhanced effective mass $m^{*}$ of the corresponding quasiparticles [Noz74, Hew93a]. Experimentally, effective masses up to several hundred times the free-electron mass are observed [Ste84]. The quasiparticles are considered to emerge below a crossover region $T^{*}$, which is typically found between $\mathrm{T}=5 \mathrm{~K}$ and $\mathrm{T}=50 \mathrm{~K}$, and they form a narrow renormalized band. The local density of states $\rho\left(E_{F}\right)$ around the Fermi energy is enhanced at low temperatures $T \ll T^{*}$ and shows a distinct peak. As outlined above, this peak results from the hybridized states with an effective bandwidth of order $T_{K}$, which is comparable to the 
Kondo-singlet binding energy.

The effective mass in HF compounds is directly proportional to the Sommerfeld coefficient $\gamma$ in the specific heat $c_{V} \approx \gamma T+\beta T^{3}$. The specific heat divided by temperature, $c_{V} / T$, is observed to decrease with cooling down to the region where $T \approx T^{*}$. There it starts to steeply increase again, which is related to the formation of the Kondo singlet an can be interpreted in a Fermi-liquid picture via an enhanced mass. Despite the many common properties of heavy-fermion compounds, the further development towards zero temperature depends on the exact material. Usually, away from quantum critical points, the specific heat $c_{V} / T$ either flattens and reaches a constant but high value for $T \rightarrow 0$ or it goes through a maximum and decreases again to a residual value.

The constant Pauli-like magnetic susceptibility for $T \ll T^{*}$ in the paramagnetic case can be interpreted as the susceptibility of heavy quasiparticles. An example for a roughly constant susceptibility for $T \rightarrow 0$ is the first $\mathrm{HF}$ compound $\mathrm{CeAl}_{3}$ discovered by Andres, Graebner and Ott in 1975 And75. Its zero-temperature value is still two orders of magnitude higher than the one of free electrons. For a Fermi liquid, the susceptibility is proportional to the density of states at the Fermi energy. Thus it is directly proportional to the effective mass and the Sommerfeld coefficient, too. This latter fact is usually expressed through the Wilson ratio $R \propto \chi / \gamma$. While this dimensionless ratio is one for free electrons, it lies between 2 and 5 in HF compounds.

The susceptibility and thermodynamic properties in most HF systems can be understood from the case of dilute magnetic impurities in a metal. The reason is their dependence on the thermal effective mass $m^{*}$, which applies as a conceptual description for the quasiparticles in the dilute case, too [Hew93b] the transition temperature $T^{*}$ in HF systems is often surprisingly close to the single-ion Kondo temperature $T_{K}$. However, the situation is more subtle for transport properties like the electrical resistivity. While in the dilute case a saturated maximum of the resistivity is observed for $T \rightarrow 0$, HF metals exhibit a maximum around the characteristic energy scale $T^{*}$ and a resistance drop towards zero temperature. This behavior can only be understood by a developing coherence. At low temperatures the quenched moments become strong scatterers, which results in an increase in resistivity. At the same time, increasing elasticity of the scattering leads to a development of phase coherence, starting to develop at roughly $T^{*}$. At an even lower temperature $T_{0} \ll T^{*}$ the scattering becomes completely coherent due to the lattice periodicity and is thereby strongly suppressed. This gives rise to a rapid resistance drop upon cooling at the onset of coherence. 


\subsection{Magnetic Order in Heavy-Fermion Materials}

In addition to the intrasite Kondo interaction between the spin-density of conduction-band electrons and the local moments, an intersite (RKKY ${ }^{1}$ ) exchange interaction among the local moments is mediated by conduction electrons. In fact, both the RKKY interaction and Kondo screening compete. The Kondo interaction tends to quench the moments and to delocalize $f$-electrons. The ratio of the corresponding two energy scales determines whether a HF groundstate is build or not. Actually, it depends on the strength of the Kondo coupling $J$ which interaction prevails. In the strong coupling regime the Kondo interaction is dominant and the HF groundstate develops. For weak coupling on the other hand, the intersite interaction wins and an antiferromagnetically ordered groundstate is formed. In principle, there are two possibilities for antiferromagnetic order in HF systems. One is that the local moments are only weakly reduced by the Kondo effect as in the prototypical $\mathrm{HF}$ antiferromagnet $\mathrm{CeAl}_{2}[\mathrm{Bre} 78$. The other is the case of itinerant spin-density-wave antiferromagnetism, which occurs among the heavy quasiparticle system with delocalized magnetic moments [Oka09]. In the region where the energy scales of both interactions are comparable, a quantum critical point (QCP) occurs at zero temperature, that is, a second-order phase transition driven by quantum fluctuations only. The observed behavior in the vicinity of a QCP deviates from a Fermi liquid; this non-Fermi liquid behavior is characterized by anomalous critical exponents, see, e.g., [Loe07].

The coupling constant $J$ can be tuned by hydrostatic pressure or chemical doping. Thereby, an initially antiferromagnetic compound can be tuned to a paramagnetic state. At zero temperature this tuning defines an antiferromagnetic quantum critical point, where the fluctuations of the magnetic order parameter diverge. In some HF systems, the QCP is actually hidden by a superconducting dome. The most prominent example of such a compound is $\mathrm{CeCu}_{2} \mathrm{Si}_{2}$, which crystallizes in a tetragonal $\mathrm{ThCr}_{2} \mathrm{Si}_{2}$ structure, cf. (Fig. 1.2). The corresponding tetragonal crystal field splits the $S=5 / 2$ multiplet of the $\mathrm{Ce}^{3+}$-ions into a lowest-lying Kramers doublet and two excited doublets around $30 \mathrm{meV}$. Hence, at low temperatures the conduction electrons effectively interact at every Ce-site with a localized spin- $1 / 2$ of the $4 f$-level, i.e., it is a typical Kondo-lattice compound. The corresponding single-ion Kondo temperature lies roughly at $T_{K}=15 \mathrm{~K}$ [Ste12a]. Both the antiferromagnetic phase and the superconducting dome above the AF QCP are found in $\mathrm{CeCu}_{2} \mathrm{Si}_{2}$. An example for a pressure-induced QCP and emerging phases at low temperatures is shown in (Fig. 1.3) for 10-at.\%-Ge-doped $\mathrm{CeCu}_{2} \mathrm{Si}_{2}$. In fact, a second superconducting dome is found in the high-pressure regime, which makes this compound a very interesting heavy-fermion superconductor. For pure $\mathrm{CeCu}_{2} \mathrm{Si}_{2}$ both domes merge and cannot be distinguished.

1 The RKKY interaction is named after Ruderman, Kittel, Kasuya and Yosida [Rud54, Kas56, Yos57. 


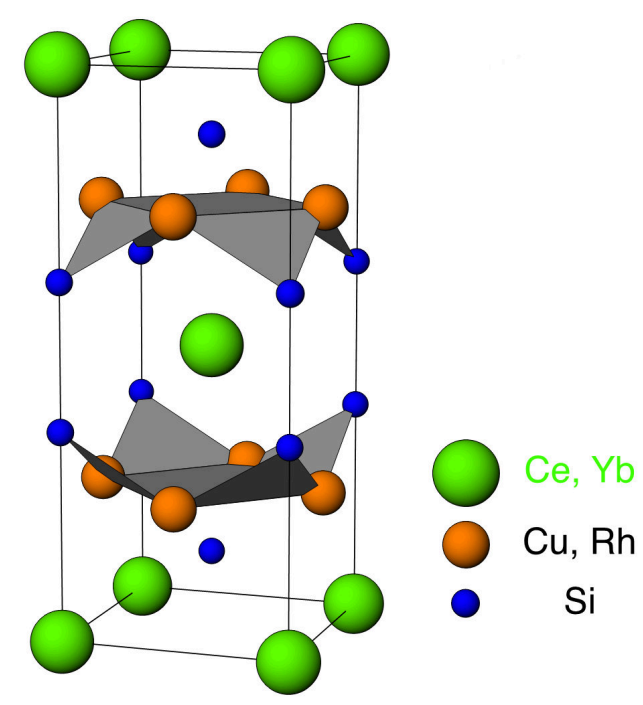

Figure 1.2: The body-centered tetragonal unit cell of the $\mathrm{ThCr}_{2} \mathrm{Si}_{2}$ structure of $\mathrm{CeCu}_{2} \mathrm{Si}_{2}$. Adapted from Ste12b.

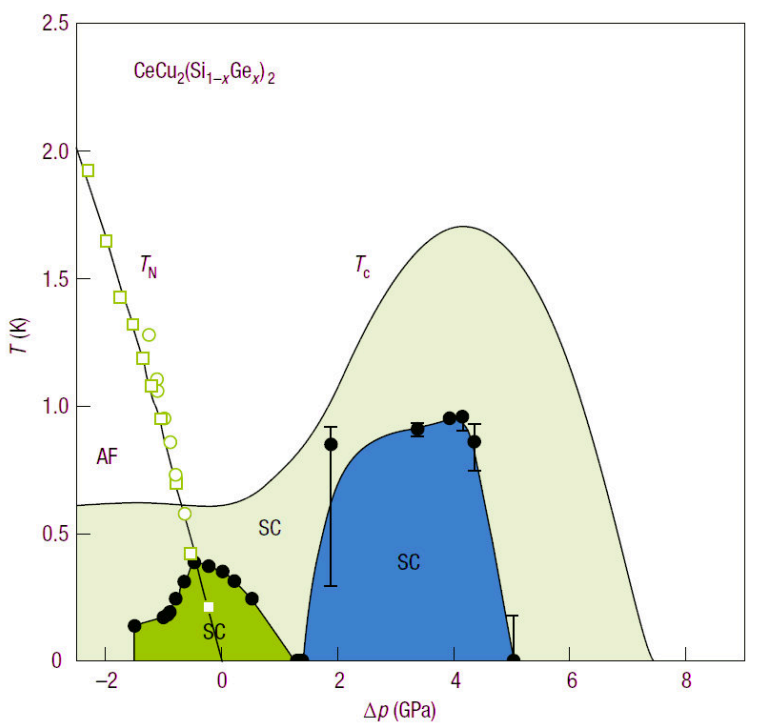

Figure 1.3: Pressure dependence of 10-at.\%Ge-doped $\mathrm{CeCu}_{2} \mathrm{Si}_{2}$. Transition temperatures for Néel- $\left(T_{N}\right)$ and superconducting $\left(T_{c}\right)$ order are shown. The thin solid $T_{c}$-line is for pure $\mathrm{CeCu}_{2} \mathrm{Si}_{2}$. Figure adapted from Ref. Geg08 with permission of P. Gegenwart.

\subsection{Heavy-Fermion Superconductors: The Case of $\mathrm{CeCu}_{2} \mathrm{Si}_{2}$}

Before the discovery of HF superconductors, magnetism and superconductivity were thought to be mutually exclusive. Dilute magnetic impurities lead to spin-flip scattering which breaks up $s$-wave singlet Cooper pairs as they are formed by an attractive interaction due to phonons. The discovery of superconductivity in $\mathrm{CeCu}_{2} \mathrm{Si}_{2}$ in 1979 by Steglich et al. Ste79] was thus quite astonishing. The dense lattice of magnetic moments in the Ce- $4 f$-shell does not only coexist with superconductivity below $0.6 K$ - it turned out that it is, in fact, a prerequisite for superconductivity in this material. Hence, the superconducting state has to be unconventional to a certain degree since the Cooper-pairs consist of heavy quasiparticles. Large deviations from standard theories of superconductivity like BCS theory [Bar57a, Bar57b] or Eliashberg theory [Eli69] were measured, hinting already to a possible non-phononic origin of Cooper pairing. Several other superconducting HF compounds like $\mathrm{CeIn}_{3}, \mathrm{UPt}_{3}$ or $\mathrm{UBe}_{13}$, which were found afterwards, do exhibit an unconventional superconducting state, too.

That the superconducting state in $\mathrm{CeCu}_{2} \mathrm{Si}_{2}$ evolves out of the heavy Fermi liquid is supported by a large amount of experimental evidence. A first hint is given by the 
compound $\mathrm{LaCu}_{2} \mathrm{Si}_{2}$ which does not become superconducting down to the milli-Kelvin range [Ste79]. Compared to $\mathrm{CeCu}_{2} \mathrm{Si}_{2}$, there is just the $4 f$-electron and thus the local moment missing. In addition, the coherence length $\xi_{c}$ and London penetration depth $\lambda_{L}$ observed in $\mathrm{CeCu}_{2} \mathrm{Si}_{2}$ are both in the range of extreme type-II superconductors - their large $\lambda_{L}$ and reduced $\xi_{c}$ stem from the strongly enhanced effective electron mass, thus indicating that the $f$-moments are involved in superconductivity [Gre91]. Apart from a few exceptions, this is true for most HF superconductors where Cooper pairing is hence attributed to the heavy-quasiparticle system.

Along with the fact that "electron-phonon coupling in $\mathrm{CeCu}_{2} \mathrm{Si}_{2}$ is not retarded, i.e., the heavy charge carriers cannot escape their own polarization cloud" [Ste12a], the involvement of $f$-moments in superconductivity hints towards a non-phononic pairing mechanism. In fact, a magnetic origin of the superconducting "glue" was proposed [Miy86, Sca86]. These early proposals have been experimentally supported in the recent years. An overdamped dispersive antiferromagnetic excitation mode has been identified to be the driving force of superconductivity in $\mathrm{CeCu}_{2} \mathrm{Si}_{2}$ Sto11. The mode coupling of these excitations to the heavy fermions is strongly retarded, as opposed to the almost non-retarded phonons. The microscopical origin of the Cooper-pair glue is thought to be related to the symmetry of the gap function or order parameter. From an anisotropic temperature dependence of the magnetic-field penetration depth [Bro90] in $\mathrm{UPt}_{3}$ or the four-fold anisotropy of the upper critical field $H_{c, 2}$ in $\mathrm{CeCu}_{2} \mathrm{Si}_{2}$ it is inferred that the order parameter in $\mathrm{HF}$ systems is anisotropic, too. In addition, non-exponential temperature dependencies of the specific heat and similar properties hint towards a $d$-wave symmetry of the gap function in $\mathrm{CeCu}_{2} \mathrm{Si}_{2}$. The ratio of the gap width $\Delta$ to the critical temperature $T_{c}$ is universal for BCS superconductors and has a value of $\Delta / T_{c} \approx 1.74$. However, this value is often exceeded in HF superconductors, which is typically interpreted in terms of weak-coupling $d$-wave pairing. At least in $\mathrm{CeCu}_{2} \mathrm{Si}_{2}$, the main driving force of superconductivity seems to be experimentally identified in the meantime ${ }^{1}$ : Non-local antiferromagnetic quantum-critical fluctuations mediate the retarded $d$-wave pairing [Sto11].

\subsection{Outline}

$\mathrm{CeCu}_{2} \mathrm{Si}_{2}$ and many other heavy-fermion systems constitute Kondo lattices, where effectively a spin- $1 / 2$ is coupled via an antiferromagnetic exchange interaction $J$ to each lattice site. Neglecting all other rather complex aspects, the essential low-temperature behavior of these HF systems is captured by the simple Kondo-lattice model (KLM). The experimental findings naturally raise the question whether also the rather complex physics can already be captured by a simple model like the KLM - especially ordered phases are of interest.

1 This statement refers to the superconducting pairing close to the AF QCP. 
It is known that the KLM naturally exhibits antiferromagnetic Néel order for a small coupling constant. However, the question whether superconductivity also emerges in the KLM without adding an extra pair-term to the model, had been remained unclear. In the present thesis, this very question is investigated along with a possible connection to magnetic properties.

The thesis is structured as follows. First, an introduction to theoretical concepts and computational tools, which can be used to solve lattice models for HF systems, is given in chapter 2. The dynamical mean-field theory (DMFT) is combined with the numerical renormalization group (NRG) method and it is described how these methods can be extended to tackle symmetry-broken phases like antiferromagnetism or $s$-wave superconductivity. In chapter 3 the low energy properties of the Kondo-lattice model (KLM) and its extensions are discussed for the paramagnetic phase applying the DMFT+NRG method. This numerical approach is afterwards applied to the antiferromagnetic phase of the KLM, where the focus is on spectral properties and the actual nature of the antiferromagnetic state. The next chapter 5 is dedicated to the surprising finding of a stable $s$-wave superconducting phase in the KLM. Some of the results presented there are published in [Bod13. Finally, in chapter 6, results for the superconducting phase of the KLM width additional interactions like the Coulomb repulsion and a local electron-phonon coupling are presented. The influence of a higher local-moment spin $S=1$ is also briefly discussed. In the very end, the results of this thesis are summarized and several conclusions are drawn, which provide the basis for an outlook to still open questions. 


\section{Computational Methods}

Except for a few limiting cases, even the simplest lattice models for strongly correlatedelectron systems constitute a rather challenging task to theoretical physics. While exact analytical solutions are often limited to one dimension, for two- or three-dimensional problems one needs to introduce certain approximations; most often the problem is then solved numerically. At present there are several methods available to tackle the quantum many-body problem in an approximative way. All these methods have their own specific advantages and drawbacks. A rather successful approach is the dynamical mean-field theory (DMFT) and its cluster extensions [Geo96, Mai05]. The DMFT maps the lattice problem to a quantum impurity problem in an effective medium, and the medium is determined self-consistently. The quantum impurity problem needs to be solved in every iteration of the DMFT self-consistency cycle. For this task one has several methods at hand, also depending on the cluster size. Here, the focus is on the combination of single-site DMFT with the numerical renormalization group (NRG) method Wil75] - it is well-suited for the treatment of low temperatures and low-energy scales as they appear in HF systems. A review on more recent developments in the field of the NRG method can be found in Bul08. The expense of DMFT methods is the loss of information about non-local correlations beyond the spatial extent of the impurity cluster, i.e., in the single-site method, non-local correlations cannot be incorporated at all. Nevertheless, it allows to include phases with broken-symmetry to a certain extent, e.g., ferromagnetism, commensurate antiferromagnetism or $s$-wave superconductivity.

This chapter is structured as follows. First, the DMFT is introduced and the inclusion of ordered phases is briefly described. Afterwards it shown how the effective single-site problem is linked to quantum impurity models. A special emphasis is put on the treatment of superconductivity. Finally, the basic concepts of the NRG method are discussed, again with an emphasis on superconductivity. Technical details of the implementation are postponed to the appendix. 


\subsection{Dynamical Mean-Field Theory}

The philosophy behind DMFT is to approximate interactions by a static spatial mean-field while the quantum fluctuations in time are retained. This is best illustrated by deriving the DMFT equations by means of the so-called cavity method [Geo96]: Utilizing a path integral formulation, spatial degrees of freedom can be integrated out and one obtains an effective single impurity problem in a dynamical mean-field, while the latter needs to be determined self-consistently. The whole DMFT procedure and the derivation of self-consistency equations is outlined in the following.

\subsubsection{Cavity Method and Effective Action}

A general one-band lattice Hamiltonian with only local interactions reads

$$
H=-\sum_{i j \sigma} t_{i j \sigma} c_{i \sigma}^{\dagger} c_{j \sigma}+\sum_{i} H_{i}^{\mathrm{loc}}
$$

where $t_{i j}$ denotes the tunneling matrix element or "hopping" between lattice sites $i$ and $j$, $\sigma$ denotes the spin, $c_{i \sigma}^{(\dagger)}$ is the creation (annihilation) operator and $H_{i}^{\text {loc }}$ gathers all local interactions that shall be included. In fact, these interactions can be rather arbitrary as long as they are local and can be handled by the impurity solver. Typical examples are a local Coulomb term, Holstein phonon modes or local spin interactions. It is also possible to add electron bands and local interband interactions. However, for the sake of simplicity, these additional terms and indices are not included here.

For the following derivation it is suitable to introduce Grassmann fields $\left\{c_{i}(\tau), \bar{c}_{i}(\tau)\right\}$ as function of imaginary time $\tau$. The corresponding functions of Grassmann fields are denoted by curly letters. Moreover, spin indices are mostly suppressed in the following, but a sum over $\sigma$ is implied. The action for the Hamiltonian (2.1) then reads

$$
\mathcal{S}\left[\left\{c_{i}, \bar{c}_{i}\right\}\right]=\int_{0}^{\beta} \mathrm{d} \tau\left(\sum_{i j} \bar{c}_{i}\left[\left(\partial_{\tau}-\mu\right) \delta_{i j}-t_{i j}\right] c_{j}+\sum_{i} \mathcal{H}_{i}^{\text {loc }}\left(\bar{c}_{i}, c_{i}\right)\right)
$$

It can be split into three different parts, $\mathcal{S}=\mathcal{S}_{o}+\mathcal{S}_{\Delta}+\mathcal{S}^{(o)}$, where the index $o$ tags an arbitrary but fixed site of the lattice. The local on-site part $\mathcal{S}_{o}$ is separated from the lattice part $\mathcal{S}^{(o)}$ which denotes the contribution from the lattice without site $o$. It is only the hopping term $t_{i j}$ which connects both parts and enters the hybridization term $\mathcal{S}_{\Delta}$. These 
three parts of the action are written as

$$
\begin{aligned}
& \mathcal{S}_{o}=\int_{0}^{\beta} \mathrm{d} \tau\left(\bar{c}_{o}\left(\partial_{\tau}-\mu\right) c_{o}+\mathcal{H}_{o}^{\mathrm{loc}}\left(\bar{c}_{o}, c_{o}\right)\right) \\
& \mathcal{S}^{(o)}=\int_{0}^{\beta} \mathrm{d} \tau\left(\sum_{i \neq o, j \neq o} \bar{c}_{i}\left[\left(\partial_{\tau}-\mu\right) \delta_{i j}-t_{i j}\right] c_{j}+\sum_{i \neq o} \mathcal{H}_{i}^{\mathrm{loc}}\left(\bar{c}_{i}, c_{i}\right)\right) \\
& \mathcal{S}_{\Delta}=-\int_{0}^{\beta} \mathrm{d} \tau \sum_{i \neq o} t_{i o}\left(\bar{c}_{i} c_{o}+\bar{c}_{o} c_{i}\right) .
\end{aligned}
$$

The principle of the cavity method is illustrated in (Fig. 2.1) and (Fig. 2.2). In order to obtain a mean field, the lattice degrees of freedom need to be integrated out. To this end, the expectation value of an operator $A$ with respect to the action $\mathcal{S}^{(o)}$ is defined as

$$
\begin{aligned}
\langle A\rangle^{(o)} & =\left(\int \prod_{\alpha} \mathcal{D}\left(\bar{c}_{\alpha}, c_{\alpha}\right) \mathrm{e}^{-\mathcal{S}^{(o)}} A\left(\bar{c}_{\alpha}, c_{\alpha}\right)\right) /\left(\int \prod_{\alpha} \mathcal{D}\left(\bar{c}_{\alpha}, c_{\alpha}\right) \mathrm{e}^{-\mathcal{S}^{(o)}}\right) \\
& =\frac{1}{\mathcal{Z}^{(o)}} \int \prod_{\alpha} \mathcal{D}\left(\bar{c}_{\alpha}, c_{\alpha}\right) \mathrm{e}^{-\mathcal{S}^{(o)}} A\left(\bar{c}_{\alpha}, c_{\alpha}\right)
\end{aligned}
$$

where the index $\alpha$ labels all potential quantum numbers and $\mathcal{Z}$ denotes the partition function. The functional integral measure is abbreviated by $\mathcal{D}(\bar{c}, c)=\lim _{N \rightarrow \infty} \prod_{i=1}^{N} \mathrm{~d} \bar{c}\left(\tau_{i}\right) \mathrm{d} c\left(\tau_{i}\right)$ for the $N$ time slices $\tau_{i}$ in the imaginary-time interval $[0, \beta]$.

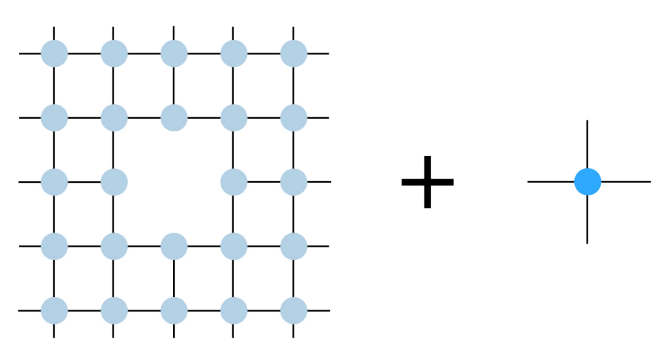

Figure 2.1: Cavity method: The lattice is divided into one site and the rest of the lattice. The left part (o) can be integrated out formally.

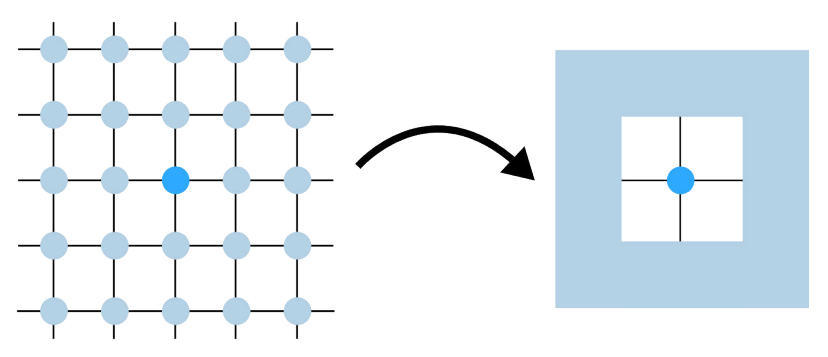

Figure 2.2: The lattice problem is mapped onto an effective single site interacting with a dynamical mean field which captures the influence of all neighboring sites. 
With these definitions at hand it is possible to integrate out the lattice part in the partition function,

$$
\begin{aligned}
\mathcal{Z} & =\int \mathcal{D}\left(\bar{c}_{o}, c_{o}\right) \mathrm{e}^{-\mathcal{S}_{o}} \int \prod_{i \neq o} \mathcal{D}\left(\bar{c}_{i}, c_{i}\right) \mathrm{e}^{-\mathcal{S}^{(o)}} \mathrm{e}^{-\mathcal{S}_{\Delta}} \\
& =\mathcal{Z}^{(o)} \int \mathcal{D}\left(\bar{c}_{o}, c_{o}\right) \mathrm{e}^{-\mathcal{S}_{o}}\left\langle\mathrm{e}^{-\mathcal{S}_{\Delta}}\right\rangle^{(o)} .
\end{aligned}
$$

By comparison, an effective action for the site $o$ can be obtained via

$$
\frac{1}{\mathcal{Z}_{\text {eff }}} \mathrm{e}^{-\mathcal{S}_{\text {eff }}\left(\bar{c}_{o}, c_{o}\right)}=\frac{1}{\mathcal{Z}} \int \prod_{i \neq o} \mathcal{D}\left(\bar{c}_{i}, c_{i}\right) \mathrm{e}^{-\mathcal{S}\left(\bar{c}_{i}, c_{i}\right)}
$$

i.e. one needs to expand the expectation value

$$
\left\langle\mathrm{e}^{-\mathcal{S}_{\Delta}}\right\rangle^{(o)}=1-\int_{0}^{\beta} \mathrm{d} \tau\left\langle\Delta_{\mathcal{S}}(\tau)\right\rangle^{(o)}+\frac{1}{2 !} \int_{0}^{\beta} \int_{0}^{\beta} \mathrm{d} \tau_{1} \mathrm{~d} \tau_{2}\left\langle\mathcal{T}_{\tau} \Delta_{\mathcal{S}}\left(\tau_{1}\right) \Delta_{\mathcal{S}}\left(\tau_{2}\right)\right\rangle^{(o)}-\ldots
$$

with the shorthand $\Delta_{\mathcal{S}}(\tau)$ for the integrand in Eq. 2.5 and with the time ordering operator $\mathcal{T}_{\tau}$. The expectation values with an odd number of operators in this series vanish, thus the lowest order term is of order two and contains the sum over unconnected 2-point Green's functions $\left\langle\mathcal{T}_{\tau} c_{i \sigma}\left(\tau_{1}\right) \bar{c}_{j \sigma}\left(\tau_{2}\right)\right\rangle^{(o)}$ since the on-site operators $c_{o}$ are not affected by the cavity average $\langle\cdot\rangle^{(o)}$.

In higher orders the $n$-th order term similarly contains $2 n$-point unconnected Green's functions. Due to the linked cluster theorem, see, e.g., [Mah00, the effective action can be expanded in terms of connected $2 n$-point functions as

$$
\mathcal{S}_{\text {eff }}=\mathcal{S}_{o}+\sum_{n=1}^{\infty} \sum_{i_{1}, \ldots, i_{n} ; j_{1}, \ldots, j_{n}} \int \mathrm{d} \tau_{1} \ldots \mathrm{d} \tau_{n}\left(\bar{J}_{i_{1}} \ldots \bar{J}_{i_{n}} G_{i_{1}, \ldots, j_{n}}^{(o), \text { linked }}\left(\tau_{i_{1}}, \ldots, \tau_{j_{n}}\right) J_{j_{1}} \ldots J_{j_{n}}\right)
$$

with the source terms $J_{i_{k}}=t_{o i_{k}} c_{i_{k}}$.

\subsubsection{The Mean-Field Limit of Infinite Dimensions}

In a lattice it is quite illustrative that a mean-field description is better when more nearestneighbor fields superimpose to have an averaged influence at a single lattice site. For example, in a fcc-lattice in three dimensions the number of nearest neighbors $z=12$ is already quite large. In the theoretical limit of infinite dimensions or, equivalently, of infinite coordination number $z \rightarrow \infty$, a mean-field theory in classical statistical mechanics gets exact; the same is true for DMFT. For $z \rightarrow \infty$, the influence of the cavity lattice " $(o)$ " on a single lattice site " $O$ " is captured by a single dynamical mean-field. All local terms of 
the Hamiltonian are still well-defined in this very limit. However, the hopping term would produce a diverging kinetic energy, as the number of bonds to adjacent sites grows linearly with increasing $z$. The kinetic energy per lattice site would scale proportional to $z$, which needs to be compensated. One finds that the hopping has to be scaled as Met89]

$$
t_{i j}=\frac{t_{i j}^{*}}{\sqrt{z_{|i-j|}}}
$$

where $|i-j|$ is the minimal distance between sites $i$ and $j$ with respect to the $\mathcal{L}_{1}$-norm, i.e., $z_{1}$ is the coordination number for nearest neighbors, $z_{2}$ for next-nearest neighbors and so on. The exact relation between $z$ and $d$ depends on the underlying lattice geometry, e.g., $z_{|i-j|}=d^{|i-j|}$ in the easiest case of the Bethe lattice, which is introduced below. As a result of Eq. 2.12) it turns out that the Green's functions $G_{i j}$ scale with the same factor $1 / \sqrt{z|i-j|}$. Thus, the kinetic energy is constant for $z \rightarrow \infty$. The scaling in this limit has the tremendous effect that all non-local contributions to the self-energy vanish [Met89],

$$
\Sigma_{i j}(\omega) \stackrel{d \rightarrow \infty}{\longrightarrow} \delta_{i j} \Sigma(\omega)
$$

which can be shown in several ways. One possibility is to take a look at the scaling of Green's functions entering the effective action in the expression Eq. (2.11). The $n$-th order term brings in a factor $(1 / d)^{n-2}$, so that only the second order term survives in the limit of infinite dimensions Geo96. Then, the effective action Eq. 2.11) extremely simplifies to

$$
\mathcal{S}_{\text {eff }}=\mathcal{S}_{o}-\sum_{i j} \int_{0}^{\beta} \int_{0}^{\beta} \mathrm{d} \tau_{1} \mathrm{~d} \tau_{2} \bar{c}_{o}\left(\tau_{1}\right) t_{o i} G_{i j}^{(o)}\left(\tau_{1}, \tau_{2}\right) t_{j o} c_{o}\left(\tau_{2}\right)
$$

Below it is shown that this action is formally equal to the effective action for an Anderson impurity model. Here, it can already be seen that the only field entering this expression belongs to the site $o$ and the rest of the one-particle terms plays the role of an effective bath. In the form

$$
\mathcal{S}_{\text {eff }}=\mathcal{S}_{\text {localint }}-\int_{0}^{\beta} \int_{0}^{\beta} \mathrm{d} \tau_{1} \mathrm{~d} \tau_{2} \bar{c}_{o}\left(\tau_{1}\right) \mathcal{G}_{0}^{-1}\left(\tau_{1}-\tau_{2}\right) c_{o}\left(\tau_{2}\right)
$$

the physical content of this effective bath becomes more apparent. While all local interactions are encoded in $\mathcal{S}_{\text {localint }}$, the effective Weiss field $\mathcal{G}_{0}$ yields the amplitude of a particle hopping at a time $\tau_{1}$ from the bath onto the site $o$ and being destroyed at a later time $\tau_{2}{ }^{1}$

1 In principle, it is also possible to take into account superconductivity and Cooper pairs, which can also 'hop' from the bath onto the impurity and back. 


\subsubsection{Self-Consistency Equations}

To obtain a closed set of self-consistency equations, the cavity Green's function $G^{(o)}$ needs to be related to the rest of the lattice by [Hub64, Geo96]

$$
G_{i j}^{(o)}=G_{i j}-G_{i o} G_{o}^{-1} G_{o j}
$$

The effective action in Eq. (2.15) is transformed in two steps. First the usual transformation from imaginary time to Matsubara frequencies is carried out, and subsequently the analytic continuation $i \omega_{n} \rightarrow \omega+i 0^{+}$is applied, so that the Weiss filed or effective medium finally reads

$$
\mathcal{G}_{0}^{-1}\left(\omega+i 0^{+}\right)=\omega+i 0^{+}+\mu-\sum_{i j} t_{o i} t_{j o} G_{i j}^{(o)}\left(\omega+i 0^{+}\right)
$$

A Fourier transform of $G_{i j}$ in the lattice sites, $t_{i j} \rightarrow \varepsilon_{\mathbf{k}}$, yields

$$
G_{\mathbf{k}}(\omega)=\left(\zeta\left(\omega+i 0^{+}\right)-\varepsilon_{\mathbf{k}}\right)^{-1}
$$

with $\zeta\left(\omega+i 0^{+}\right)=\omega+i 0^{+}+\mu-\Sigma\left(\omega+i 0^{+}\right)$and a local self-energy $\Sigma\left(\omega+i 0^{+}\right)$. Using the relations $\sum_{\mathbf{k}} \varepsilon_{\mathbf{k}} G_{\mathbf{k}}(z)=-1+\zeta G_{o}(z)$ and $\sum_{\mathbf{k}} \varepsilon_{\mathbf{k}}^{2} G_{\mathbf{k}}(z)=-\zeta+\zeta^{2} G_{o}(z)^{2}$, one obtains the central self-consistency equation

$$
\mathcal{G}_{0}^{-1}\left(\omega+i 0^{+}\right)=\Sigma\left(\omega+i 0^{+}\right)+G_{o}\left(\omega+i 0^{+}\right)^{-1}
$$

which relates the effective medium with the impurity Green's function. It is formally similar to the Dyson equation but of different physical content.

How does this equation now allow for a self-consistent solution? They key ingredient is the calculation of the local Green's function for the site $o$ by $k$-summation of the non-interacting propagator $G_{\mathbf{k}, 0}(z)$,

$$
G_{o}(z)=\sum_{\mathbf{k}}\left(G_{k, 0}^{-1}(z)-\Sigma(z)\right)^{-1}
$$

For a given self-energy it is thereby possible to determine the effective medium by means of Eq. 2.17). In the practical implementation one can start with, e.g., $\Sigma=0$ or with another initial guess of $\Sigma(z)$. The resulting Weiss field $\mathcal{G}_{0}(z)$ defines the effective impurity problem which needs to be solved by an appropriate impurity solver. This, in turn, yields a new impurity Green's function. The latter can be put into Eq. (2.17), this time solved 
for $\Sigma$, so as to obtain a new self-energy. ${ }^{1}$ At this point, the whole procedure needs to be iterated, as illustrated in (Fig. 2.3), until convergence is reached.

The $\mathbf{k}$-summation in Eq. 2.18) can be replaced by an integral over $\varepsilon$ which constitutes a Hilbert transform $\mathcal{H}$ of the non-interacting density of states $\rho_{0}(\omega)$ :

$$
G_{o}(z)=\sum_{\mathbf{k}}\left(G_{k, 0}^{-1}(z)-\Sigma(z)\right)^{-1}=\int \mathrm{d} \varepsilon \rho_{0}(\varepsilon)(\zeta-\varepsilon)^{-1}=: \mathcal{H}\left[\rho_{0}\right](\zeta)
$$

The self-consistency equations above have been derived for the paramagnetic case, but it is possible to extent them to symmetry-broken phases, namely ferromagnetism, antiferromagnetism and superconductivity Geo96]. The latter two are of high interest with respect to heavy fermions and the corresponding extension of the DMFT is explained in the following two subsections.

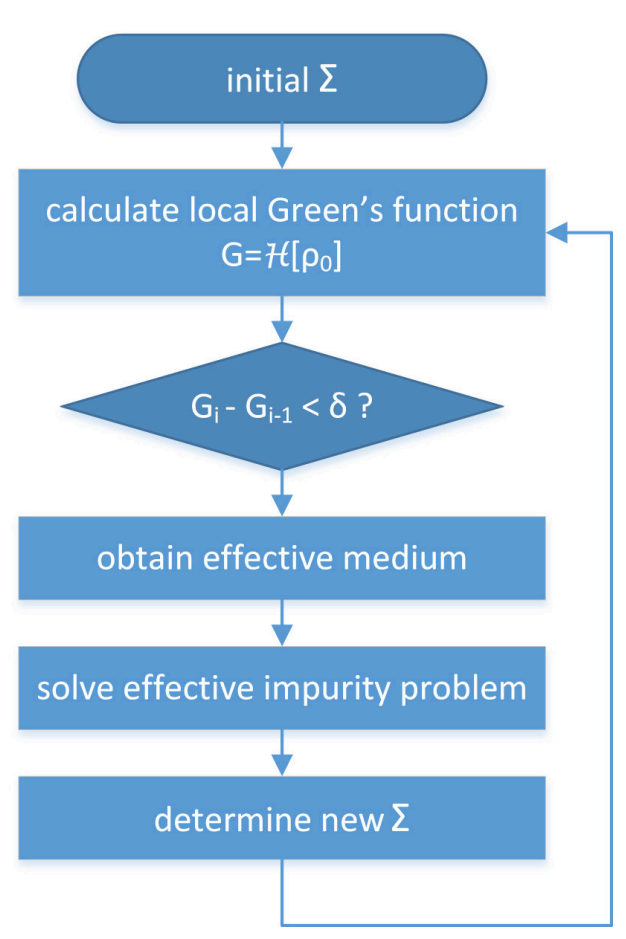

Figure 2.3: The DMFT self-consistency loop.

1 In practice, it is more convenient to use the so-called self-energy trick [Bul98 and compute $\Sigma(z)$ as the ratio of higher Green's functions, see section 2.4.5. 


\subsubsection{Antiferromagnetism}

In the derivation above, spin-indices have been mostly suppressed since the focus has been on the paramagnetic state. The SU(2) symmetry of the Hamiltonian does then not allow for magnetically ordered phases. Thus, one needs to carry out all calculations in a spin-dependent way. The Néel state is the simplest antiferromagnetically ordered state where the spin directions simply alternate on the lattice sites. To include Néel order, a bipartite $A$ - $B$-structure of the lattice must be considered in the DMFT: Each $A$-site has only $B$-sites as nearest neighbors and vice versa. The whole lattice thus consists of two sublattices. Neighboring sites $A$ and $B$ have different self-energies [Geo96], but in the Néel state the relation between them simply reads

$$
\Sigma_{\sigma}^{A}=\Sigma_{\bar{\sigma}}^{B},
$$

for opposite spins $\sigma$ and $\bar{\sigma}$. For this reason, one needs to solve only one effective impurity problem; interchanging the spin indices yields the solution for the other sublattice. The hopping occurs exclusively between $A$ - and $B$-sublattices, so that the local Green's function written in the $A, B$-basis reads

$$
\mathbf{G}_{o, \sigma}(\omega)=\int \mathrm{d} \varepsilon \rho_{0}(\varepsilon)\left(\begin{array}{cc}
\zeta_{\sigma}^{A} & -\varepsilon \\
-\varepsilon & \zeta_{\sigma}^{B}
\end{array}\right)^{-1},
$$

with $\zeta_{\sigma}^{A / B}=\omega+i 0^{+}+\mu-\Sigma_{\sigma}^{A / B}(z)$. With this equation the effective fields can now be calculated for both spin directions and sublattices. In the actual implementation of DMFT it is necessary to break the $\mathbf{S U}(2)$ symmetry in the first iteration by applying a small magnetic field $\zeta_{\sigma}^{A} \rightarrow \zeta_{\sigma}^{A}+\sigma h$ and $\zeta_{\sigma}^{B} \rightarrow \zeta_{\sigma}^{B}-\sigma h$. Thereby, non-zero differences between the two sublattices are induced, which do not necessarily evolve out of numerical noise only. After the very first iteration the field is switched off again and the self-consistency cycle continues as usual. If the system is unstable towards antiferromagnetism, it evolves into a self-consistent solution with $\Sigma_{\uparrow}^{A / B} \neq \Sigma_{\downarrow}^{A / B}$ and a finite polarization $\left\langle s_{z}\right\rangle=\left(n_{\uparrow}-n_{\downarrow}\right) / 2$.

\subsubsection{Superconductivity}

The extension of DMFT equations to superconducting long-range order is a bit more involved since anomalous Green's functions $\left\langle\left\langle c_{\mathbf{k} \uparrow} ; c_{-\mathbf{k} \downarrow}\right\rangle\right\rangle$ need to be accounted for. To do so, it is convenient to introduce the Nambu formalism [Nam60] which gathers particle and hole operators in a single two-component spinor

$$
C_{\mathbf{k}}^{\dagger}=\left(c_{\mathbf{k} \uparrow}^{\dagger}, c_{-\mathbf{k} \downarrow}\right), \quad C_{\mathbf{k}}=\left(\begin{array}{c}
c_{\mathbf{k} \uparrow} \\
c_{-\mathbf{k} \downarrow}^{\dagger}
\end{array}\right) .
$$


Nambu spinors obey the usual fermionic anti-commutation rules ${ }^{1}\left[C_{\mathbf{k}}, C_{\mathbf{k}^{\prime}}^{\dagger}\right]_{+}=\delta_{\mathbf{k k}^{\prime}} \mathbf{1}_{2}$ and $\left[C_{\mathbf{k}}, C_{\mathbf{k}^{\prime}}\right]_{+}=0$ with the identity matrix $\mathbf{1}_{2}$. The complete derivation of DMFT equations can be rewritten in the Nambu formalism, with a generalized self-energy

$$
\Sigma(\omega)=\left(\begin{array}{cc}
\Sigma_{11}(\omega) & \Sigma_{12}(\omega) \\
\Sigma_{12}(-\omega)^{*} & -\Sigma_{11}(-\omega)^{*}
\end{array}\right)
$$

where the off-diagonal parts contain information about the frequency-dependence of electron pairing. The particle and hole components of both $\mu$ and $\varepsilon$ have opposite sign, which needs to be taken care of. The local Green's function reads

$$
\mathbf{G}(\omega)=\int \mathrm{d} \varepsilon \rho_{0}(\varepsilon)\left(\begin{array}{cc}
\left(\omega+i 0^{+}\right)+\mu-\varepsilon-\Sigma_{11}(\omega) & -\Sigma_{12}(\omega) \\
-\Sigma_{12}(-\omega)^{*} & \left(\omega+i 0^{+}\right)-\mu+\varepsilon+\Sigma_{11}(-\omega)^{*}
\end{array}\right)^{-1}
$$

so that an anomalous component in the self-energy induces a finite off-diagonal part $\mathcal{G}_{0}^{\text {off }}(\omega)$ in the effective medium, too. In the corresponding effective action, a term like $c_{o}^{\dagger}\left(\tau_{1}\right)\left[\mathcal{G}_{0}^{\text {off }}\left(\tau_{1}-\tau_{2}\right)\right]^{-1} c_{o}^{\dagger}(\tau)$ survives. This term illustrates the physics encoded in the anomalous part of the effective medium: $\mathcal{G}_{0}^{\text {off }}\left(\tau_{1}-\tau_{2}\right)$ is the amplitude for the process that two particles pair up on the local site - one particle coming from the bath onto the local site at a time $\tau_{1}$ and a second at a latter time $\tau_{2}$. The local pairing can thus be strongly retarded. Quantum fluctuations in imaginary time can mediate this pairing. The impurity solver has to be able to include such an anomalous part of the effective medium, too. Due to the frequency dependence, this task is highly non-trivial. The way to implement it in the NRG is shown in appendix B.

For the Hilbert transform in Eq. $(2.22)$ it is very convenient to use a partial fraction decomposition of the integrand. Thereby, it is possible to express the resulting terms as linear combinations of the usual Hilbert transform $\mathcal{H}\left[\rho_{0}\right]$. This also holds for a combined $A-B$ - and Nambu-formalism. The explicit calculation is shown in appendix A. Similar to the case of antiferromagnetism, it is important to break the $\mathrm{U}(1)$ symmetry $^{2}$ in the very first iteration by applying a small pair field. In this way, non-zero off-diagonal components are induced and can evolve self-consistenly. Without the initial "kick" this might not happen out of numerical noise only.

1 The (anti-) commutators are understood as component-wise commutators of the outer product of spinors.

2 A pair condensate has a well-defined phase $\phi$, which corresponds to a broken global U(1) gauge symmetry. The latter normally ensures particle number conservation being not present in a pair condensate. 


\subsection{Lattice Topologies}

The density of states entering the hilbert transform is the only quantity which contains information about the spatial structure of the original lattice. In the limit of infinite dimensions, an important example is the Bethe lattice, for which most of the calculations in this thesis are done. This rather artificial lattice can be constructed as follows. Initially, one site is connected to $z$ nearest neighbors. Every site of this first nearest-neighbor shell is then connected to $z-1$ sites and so on. A very important property resulting from this construction is that the lattice does not contain any closed loops, that is, every two sites are connected by only a single path. As a result many models can be solved exactly on the Bethe lattice [Bax08]. The corresponding local Green's function can be written as Eco90

$$
G_{o}^{-1}(z)=z-t^{2} \sum_{i \in \mathrm{NN}} G_{i i}^{(o)}(z)
$$

where $t=t_{i j}$ denotes the nearest neighbor (NN) hopping and $G^{(o)}$ is again the Green's function for the lattice with a site $o$ removed, see section 2.1.1. In principle, a secondnearest neighbor hopping can be included [Pet09], but throughout this thesis we will resort to a simple NN hopping. For the Bethe lattice with $z \rightarrow \infty$, Eq. 2.16) simplifies to $G_{i i}^{(o)}(z)=G_{i i}(z)$, thus one can apply the scaling Eq. 2.12$), t=t^{*} / \sqrt{z}$, and solve the resulting equation $G_{o}^{-1}(z)=z-t^{2} G_{o}(z)$ for $G(z):=G_{o}(z)$ :

$$
G(z)=\frac{1}{2 t^{2}}\left(z+\sqrt{4 t^{2}-z^{2}}\right) .
$$

The density of states can be derived by taking the imaginary part of the Green's function, $\rho(\varepsilon)=-\frac{1}{\pi} \operatorname{Im} G\left(\varepsilon+i 0^{+}\right)$, and reads for the Bethe lattice

$$
\rho(\varepsilon)=\frac{1}{2 t} \sqrt{4 t^{2}-\varepsilon^{2}} \quad, \quad|\varepsilon| \leq 2 t
$$

i.e., a simple semi-circular structure results for $d \rightarrow \infty$. At the band edges $\omega= \pm 2 t$ this DOS has the same analytic properties like a three dimensional cubic DOS [Eco90] - which makes it a fairly good approximation for the three-dimensional DOS. Moreover, the Bethe lattice can be divided into a bipartite structure and hence allows for a Néel state.

Below, a two-dimensional cubic lattice is used in some cases. Although the DMFT is a rather crude approximation for two-dimensional systems, the incorporation of van-Hove singularities in this case can lead to qualitatively different results. The $2 d$-DOS contains the complete elliptic integral of the first kind, $K_{1}(\varepsilon)$, and reads Eco90]

$$
\rho_{2 \mathrm{~d}-\mathrm{cubic}}(\varepsilon)=\frac{2}{\pi^{2} W} \theta(W-|\varepsilon|) K_{1}\left(\sqrt{1-\varepsilon^{2} / W^{2}}\right)
$$


A comparison of the two different cases is shown in (Fig. 2.4) and (Fig. 2.5). A twodimensional DOS is mainly used for illustrative reasons in this work, provided that the physics for both Bethe lattice and $2 d$-square lattice do not differ qualitatively. The spectral function

$$
A\left(\varepsilon_{\mathbf{k}}, \omega\right)=-\frac{1}{\pi} \operatorname{Im} G\left(\varepsilon_{\mathbf{k}}, \omega+i 0^{+}\right) \stackrel{\mathrm{DMFT}}{=}-\frac{1}{\pi} \operatorname{Im}\left[\omega+i 0^{+}+\varepsilon_{\mathbf{k}}-\Sigma(\omega)\right]^{-1}
$$

of a two-dimensional system as function of momentum $k_{x}$ and $k_{y}$ is a good starting point to compare with results from cluster methods or even experimental results from ARPES ${ }^{1}$. Moreover, a two-dimensional Fermi surface can be obtained by plotting $A\left(\varepsilon_{\mathbf{k}}, 0\right)$. One can simply use the above formula to obtain the spectral function from the self-energy calculated in DMFT. Although the self-energy is purely local in DMFT, one can reintroduce a momentum dependence via the energy dispersion $\varepsilon_{\mathbf{k}}$ of non-interacting electrons on the corresponding lattice, e.g., $\varepsilon_{\mathbf{k}}=-2 t \sum_{n=1}^{d} \cos \left(k_{n} a\right)$ for a $d$-dimensional cubic lattice with lattice constant $a$. The artificial $\mathbf{k}$-dependence does not provide any information about non-local correlations. In the case of the Bethe lattice, however, there is no such dispersion relation but one can simply plot $A(\varepsilon, \omega)$ as function of energy $\varepsilon$.

Figure 2.4: DOS of the Bethe lattice for $d=\infty$.
Figure 2.5: DOS of the $2 d-$ square lattice.

1 Angle-Resolved PhotoEmission Spectroscopy 


\subsection{Quantum Impurities and their Relation to DMFT}

Although the degrees of freedom are reduced to a single site, there exists no explicit solution for the problem stated by the effective action in Eq. (2.14). However, this single-site action can be related to a quantum impurity problem, which in turn can be solved numerically. The lattice models of interest in this thesis can all be captured by the sum of a hopping term and a term with local interactions on each site. As it is shown above, this class of models is within DMFT mapped onto an effective impurity problem with three ingredients: 1.) An on-site term including local interactions, 2.) a hybridization term which couples the impurity to an effective bath, and 3.) a Hamiltonian for the non-interacting bath degrees of freedom. These problems can be classified as Anderson impurity models (AIM) with certain on-site interactions,

$$
H_{\mathrm{AIM}}=H_{\mathrm{imp}}+H_{\mathrm{hyb}}+H_{\mathrm{band}}
$$

with

$$
\begin{aligned}
H_{\mathrm{hyb}} & =\sum_{\mathbf{k} \sigma} V_{\mathbf{k} \sigma}\left(d_{\sigma}^{\dagger} c_{\mathbf{k} \sigma}+c_{\mathbf{k} \sigma}^{\dagger} d_{\sigma}\right) \quad \text { and } \\
H_{\mathrm{band}} & =\sum_{\mathbf{k} \sigma} \varepsilon_{\mathbf{k} \sigma} c_{\mathbf{k} \sigma}^{\dagger} c_{\mathbf{k} \sigma}+\sum_{\mathbf{k}}\left(\Delta_{\mathbf{k}} c_{\mathbf{k} \uparrow}^{\dagger} c_{-\mathbf{k} \downarrow}^{\dagger}+\Delta_{\mathbf{k}}^{*} c_{-\mathbf{k} \downarrow} c_{\mathbf{k} \uparrow}\right)
\end{aligned}
$$

where the impurity operators $d_{\sigma}^{(\dagger)}$ couple via the hybridization matrix element $V_{\mathbf{k} \sigma}$ to the conduction electrons. The spin-dependence is here retained in the notation for the case of magnetic phases. Moreover, a BCS-type pairing term with a pair amplitude $\Delta_{\mathbf{k}}$ is added to allow for superconductivity. Including all prospective interactions studied in this thesis, the local impurity term reads

$$
H_{\mathrm{imp}}=\sum_{\sigma} \varepsilon_{\sigma}^{d} d_{\sigma}^{\dagger} d_{\sigma}+U n_{\uparrow}^{d} n_{\downarrow}^{d}+J \vec{S} \cdot \vec{s}^{d}+\omega_{0} b^{\dagger} b+\lambda\left(b^{\dagger}+b\right) \cdot \sum_{\sigma} d_{\sigma}^{\dagger} d_{\sigma}
$$

The on-site energy is denoted by $\varepsilon_{\sigma}^{d}$. The Hubbard- $U$ captures a local Coulomb repulsion and $J$ is the strength of an explicit exchange interaction of the local conduction-electron spin-density

$$
\vec{s}^{d}=\frac{1}{2} \sum_{\alpha, \beta} d_{\alpha}^{\dagger} \overrightarrow{\boldsymbol{\sigma}}_{\alpha \beta} d_{\beta}
$$

with a local magnetic moment $\vec{S}$ and the vector $\overrightarrow{\boldsymbol{\sigma}}$ of Pauli spin matrices. In addition, a local bosonic mode $b^{(\dagger)}$ with a frequency $\omega_{0}$ can be coupled via $\lambda$ to the local charge density.

In the following, the effective action for such an AIM in a superconducting medium is related to the effective action arising in DMFT. The most important task is to express 
the hybridization $V$ and the pair potential $\Delta$ of the AIM as function of the effective medium. Only then, it is possible to relate the AIM with the DMFT. Employing the Nambu formalism and using Grassmann variables again, the action for the AIM reads

$$
\mathcal{S}_{\mathrm{AIM}}=\int_{0}^{\beta} \int_{0}^{\beta} \mathrm{d} \tau_{1} \mathrm{~d} \tau_{2}\left[-\sum_{\mathbf{k}} \bar{C}_{\mathbf{k}} \mathbf{G}_{\mathbf{k}}^{-1}\left(\tau_{1}-\tau_{2}\right) C_{\mathbf{k}}+\sum_{\mathbf{k}} V_{\mathbf{k}}\left(\bar{C}_{\mathbf{k}} \boldsymbol{\tau}_{3} D+\bar{D} \boldsymbol{\tau}_{3} C_{\mathbf{k}}\right)+\mathcal{H}_{\mathrm{imp}}\right],
$$

where irrelevant constant terms as well as spin indices and some imaginary-time arguments are suppressed. The Pauli- $z$ matrix in Nambu space is denoted by $\boldsymbol{\tau}_{3} .\left(V_{\mathbf{k}}, \Delta_{\mathbf{k}}\right) \in \mathbb{R}$ is assumed in the following.

Similar to the derivation of the effective action in the DMFT, it is possible to integrate out the lattice degrees of freedom by completing the square in the partition function. The result reads

$$
\mathcal{S}_{\text {eff }}^{\mathrm{AIM}}=\int_{0}^{\beta} \int_{0}^{\beta} \mathrm{d} \tau_{1} \mathrm{~d} \tau_{2} \bar{D}\left(\tau_{1}\right) \mathcal{G}_{0}^{-1}\left(\tau_{1}-\tau_{2}\right) D\left(\tau_{2}\right)+\mathcal{S}_{\text {imp }}
$$

The Fourier transform of the effective medium is obtained as $\mathcal{G}_{0}\left(i \omega_{n}\right)=-\int_{0}^{\beta} \mathrm{d} \tau e^{i \omega_{n} \tau} \mathcal{G}_{0}(\tau-$ $0)$. In the standard approach, the Weiss field is equated to the non-interacting Green's function of the impurity,

$$
\mathbf{G}_{0}\left(i \omega_{n}\right)^{-1}=i \omega_{n} \mathbf{1}-\varepsilon^{d} \boldsymbol{\tau}_{3}-\boldsymbol{\Gamma}\left(i \omega_{n}\right)
$$

here with a generalized hybridization matrix $\boldsymbol{\Gamma}(i \omega)$ to account for superconductivity. Importantly, the AIM effective action is formally equivalent to the effective action in DMFT, Eq. 2.15). By means of the Dyson equation and the DMFT equation $\mathbf{G}_{d}^{-1}+\boldsymbol{\Sigma}=\mathcal{G}_{0}^{-1}$ one obtains the complex hybridization matrix

$$
\boldsymbol{\Gamma}\left(i \omega_{n}\right)=i \omega_{n} \mathbf{1}-\mathcal{G}_{0}^{-1}\left(i \omega_{n}\right)
$$

In the following we work with real frequencies obtained via analytic continuation $i \omega_{n} \rightarrow$ $\omega+i 0^{+}=z$. The hybridization matrix of a non-interacting AIM in a superconducting bath reads

$$
\begin{aligned}
\boldsymbol{\Gamma}(z) & =\frac{1}{N} \sum_{\mathbf{k}} \frac{V_{\mathbf{k}}^{2}}{z^{2}-\varepsilon_{\mathbf{k}}^{2}-\Delta_{\mathbf{k}}^{2}}\left(\begin{array}{cc}
z+\varepsilon_{\mathbf{k}} & \Delta_{\mathbf{k}} \\
\Delta_{\mathbf{k}} & z-\varepsilon_{\mathbf{k}}
\end{array}\right) \\
& =-\int \mathrm{d} \varepsilon \rho_{0}(\varepsilon) \frac{V(\varepsilon)^{2}}{z^{2}-\varepsilon^{2}-\Delta(\varepsilon)^{2}}\left(\begin{array}{cc}
z+\varepsilon & \Delta(\varepsilon) \\
\Delta(\varepsilon) & z-\varepsilon
\end{array}\right) .
\end{aligned}
$$

The two formulas for $\boldsymbol{\Gamma}(z)$ allow for a connection between the DMFT and the impurity 
model. The poles $\varepsilon_{ \pm}= \pm \sqrt{z^{2}-\Delta\left(\varepsilon_{ \pm}\right)^{2}}$, where in general $\Delta\left(\varepsilon_{+}\right) \neq \Delta\left(\varepsilon_{-}\right)$, can be used for a partial fraction expansion

$$
\frac{-(z+\varepsilon)}{\left(\varepsilon-\varepsilon_{+}\right)\left(\varepsilon-\varepsilon_{+}\right)}=\frac{a_{1}}{\varepsilon-\varepsilon_{-}}+\frac{a_{2}}{\varepsilon-\varepsilon_{+}}
$$

Replacing $\gamma(\varepsilon)=\pi \rho_{0}(\varepsilon) V(\varepsilon)^{2}, z \rightarrow \omega+i 0^{+}$and using the Plemelj formula $1 /\left(x+i 0^{+}\right)=$ $\mathcal{P}(1 / x)-i \pi \delta(x)$, where $\mathcal{P}$ denotes the Cauchy principal value, the integration in Eq.2.25 can be easily done for the imaginary part. Since $\operatorname{Im} \Gamma_{11}(\omega)$ does not have a defined parity, two independent equations are provided by $+\omega$ and $-\omega$ :

$$
\operatorname{Im} \Gamma_{11}( \pm \omega)=\operatorname{sgn}(\omega)\left[\frac{ \pm \omega+\varepsilon_{-}}{\varepsilon_{+}-\varepsilon_{-}} \gamma\left(\varepsilon_{-}\right)+\frac{ \pm \omega+\varepsilon_{+}}{\varepsilon_{+}-\varepsilon_{-}} \gamma\left(\varepsilon_{+}\right)\right] .
$$

In a similar way one obtains

$$
\operatorname{Im} \Gamma_{12}(\omega)=\operatorname{sgn}(\omega)\left[\frac{-\Delta\left(\varepsilon_{-}\right)}{\varepsilon_{+}-\varepsilon_{-}} \gamma\left(\varepsilon_{-}\right)-\frac{\Delta\left(\varepsilon_{+}\right)}{\varepsilon_{+}-\varepsilon_{-}} \gamma\left(\varepsilon_{+}\right)\right]
$$

The poles are given by the implicit expression above, but in the case of an even pair function $\Delta(\varepsilon)=\Delta(-\varepsilon)$, it follows that $\varepsilon_{ \pm}= \pm \varepsilon= \pm \sqrt{\omega^{2}-\Delta(\varepsilon)^{2}}$. Finally, the system of the three above equations needs to be solved (numerically) for $\{\Delta(\omega), \gamma(+\omega), \gamma(-\omega)\}$. These functions define a generalized impurity model, cf. appendix B. The quantum impurity model can be solved by means of the numerical renormalization group, which is the topic of the next section.

\subsection{Numerical Renormalization Group for Superconductors}

The renormalization group ( $R G$ ) concept was originally developed in quantum field theory [Stu53, GM54 where it provides a tool to deal with divergencies. Later, the RG idea was applied to condensed matter theory as well [Kad66, Wil75], where it is an important tool to study low-energy properties of several model systems. Renormalization links the behavior of a physical system at different energy scales: The basic idea is to successively integrate out virtual high energy fluctuations and to adapt the Hamiltonian of the system. Thereby the low-energy interactions induced by the virtual high-energy fluctuations are taken into account Col13.

The most important concept in the RG procedure is the RG transformation [Hew93b, Bul08]. In such a transformation $R_{b}$, specified by a continuous parameter $b$, a Hamiltonian $H(\vec{K})$ with a given set of coupling constants $\vec{K}=\left(K_{1}, \ldots, K_{m}\right)$ is mapped to a Hamiltonian $H\left(\vec{K}^{\prime}\right)$ of the same form but with new coupling constants $\vec{K}^{\prime}=\left(K_{1}^{\prime}, \ldots, K_{m}^{\prime}, K_{m+1}^{\prime}, \ldots\right)$, 
i.e.,

$$
R_{b}: H(\vec{K}) \longrightarrow H\left(\vec{K}^{\prime}\right), \vec{K} \longrightarrow \vec{K}^{\prime}
$$

The correlation lengths $\xi(\vec{K})$ of the system thereby scale as

$$
\xi\left(\vec{K}^{\prime}\right)=\frac{1}{b} \xi(\vec{K})
$$

The RG flow $\vec{K}_{n+1}=R_{b}\left(\vec{K}_{n}\right)$ of the coupling constants can end in fixed points $\vec{K}^{*}$, for which $R_{b}\left(\vec{K}^{*}\right)=\vec{K}^{*}$. In this case the physics of the system described by $H$ can be derived from this fixed point. In the neighborhood of an isolated fixed point the mapping can be linearized, which effectively leads to an eigenvalue problem for the corresponding linear term $T_{b}\left(\vec{K}^{*}\right)$ :

$$
T_{b} \vec{\phi}_{i}(b)=\lambda_{i}(b) \vec{\phi}_{i}(b)
$$

where $\vec{\phi}$ is a complete basis in the space of coupling constants. Linearity brings along the semi-group structure $R_{b^{\prime}}\left(R_{b}(\vec{K})\right)=R_{b b^{\prime}}(\vec{K})$ of the renormalization procedure. In addition, $\left(T_{b}\right)^{l}=T_{b^{l}}$. The expansion $\vec{K}_{n}=\sum_{i} u_{i}^{(n)} \vec{\phi}_{i}$ leads to the notion of scaling fields $u_{i}^{(n)}$ and to the relation

$$
u_{i}^{(n+l)}=\lambda_{i}^{l} u_{i}^{(n)}
$$

with which a classification of the scaling fields is possible:

- $\left|\lambda_{i}\right|>1: u_{i}$ grows and is called relevant

- $\left|\lambda_{i}\right|<1: u_{i} \longrightarrow 0$ and is called irrelevant

- $\left|\lambda_{i}\right|=1: u_{i}$ does not change and is called marginal

A stable fixed point has only irrelevant scaling fields, while one or more relevant scaling fields yield an unstable fixed point - the trajectories are finally driven away from it. Without any relevant eigenvalue or at least one marginal one, fixed points are called marginal. Except for the case of a stable fixed point, further investigations are needed to describe the behavior of the RG flow towards that fixed point.

A method to carry out the renormalization procedure numerically was established by Wilson Wil75] and applied to the Kondo impurity problem. Later, this numerical renormalization group (NRG) method was also successfully applied to the single impurity Anderson model (SIAM) [Km80a, Km80b], which is the model of interest here. A recent review of the NRG method applied to various quantum impurity problems can be found in [Bul08]. 
In most applications, the NRG is carried out in the following steps.

1. The energy support of the effective medium, or "bath", is divided into a set of logarithmic intervals.

2. Logarithmic discretization: The continuous spectrum is reduced to a discrete set of states.

3. Tridiagonalization: The discretized model is mapped onto a semi-infinite chain.

4. Iterative diagonalization of the semi-infinite chain.

Afterwards, all quantities calculated in the iterative diagonalization can be used for a further analysis of static and dynamic properties of the quantum impurity model. As the details of the NRG and its implementation are accurately described, e.g., in Ref.'s [Ž07, Bul08], here the focus is on the inclusion of superconducting correlations in the NRG approach, such that it can be combined with DMFT. Actually, the inclusion of an energy-independent pair amplitude $\Delta_{\mathrm{sc}}^{0}$ in the NRG is simple and has already been achieved early in the 1990's [Sat92, Sak93. However, for the combination with DMFT, the frequency dependence of the effective medium needs to be taken into consideration [Bul97]. The NRG thus needs to handle a non-constant pair amplitude $\Delta_{\mathrm{sc}}(\omega)$, too. The corresponding implementation is briefly sketched in the following while computational details can be found in appendix $B$.

\subsubsection{Logarithmic Discretization}

For the NRG, the band part $H_{\text {band }}$ and hybridization part $H_{\text {hyb }}$ of the Anderson-impurity Hamiltonian in Eq. (2.23) need to be generalized to a continuous energy support, which is shown in appendix B. Next, the energy support is divided in intervals $I_{m}^{ \pm}$with an exponentially decreasing width. In the interval $[-1,1]$ in terms of half the bandwidth $D$, an appropriate possibility is $I_{m}^{-}=\left[-\Lambda^{-m},-\Lambda^{-(m+1)}\right], I_{m}^{+}=\left[\Lambda^{-(m+1)}, \Lambda^{-m}\right]$ with the logarithmic discretization parameter $\Lambda>1$. In each of these intervals, a complete set of orthonormal plane-wave functions $\psi_{m l}$ is introduced and only the constant $l=0$ sates are retained, to which the impurity site couples [Wil75]. Denoting the band states corresponding to positive energies as $a_{n}^{(\dagger)}$ and the ones for negative energies as $b_{n}^{(\dagger)}$, the resulting discretized band Hamiltonian reads

$$
\begin{aligned}
H_{\mathrm{band}}= & \sum_{n}\left(\xi_{n}^{+} a_{n}^{\dagger} a_{n}+\xi_{n}^{-} b_{n}^{\dagger} b_{n}\right) \\
& +\sum_{n} \Delta_{n}^{+}\left(a_{n \uparrow}^{\dagger} a_{n \downarrow}^{\dagger}+a_{n \downarrow} a_{n \uparrow}\right)+\Delta_{n}^{-}\left(b_{n \uparrow}^{\dagger} b_{n \downarrow}^{\dagger}+b_{n \downarrow} b_{n \uparrow}\right) .
\end{aligned}
$$


A similar result is obtained for the discretized hybridization term:

$$
H_{\mathrm{hyb}}=\frac{1}{\sqrt{\pi}} d_{\sigma}^{\dagger} \sum_{n}\left(\gamma_{n}^{+} a_{n \sigma}+\gamma_{n}^{-} b_{n \sigma}\right)+\frac{1}{\sqrt{\pi}} \sum_{n}\left(\gamma_{n}^{+} a_{n \sigma}^{\dagger}+\gamma_{n}^{-} b_{n \sigma}^{\dagger}\right) d_{\sigma}
$$

The coefficients $\left\{\gamma_{n}^{ \pm}, \xi_{n}^{ \pm}, \Delta_{n}^{ \pm}\right\}$are calculated by means of the functions $\gamma(\omega)$ and $\Delta(w)$ determined from Eqns. 22.26), 2.27). A more detailed derivation and the actual calculation of the discretization coefficients is presented in appendix $B$.

\subsubsection{Tridiagonalization}

The discretized band part of the Hamiltonian can be expressed by Nambu spinors $A_{n}=$ $\left(a_{n, \uparrow}, a_{n, \downarrow}^{\dagger}\right)^{T}$ and $\boldsymbol{\Gamma}_{n}^{ \pm}$given by

$$
\boldsymbol{\Gamma}_{n}^{ \pm}=\left(\begin{array}{cc}
\xi_{n}^{ \pm} & \Delta_{n}^{ \pm} \\
\Delta_{n}^{ \pm} & -\xi_{n}^{ \pm}
\end{array}\right)
$$

which gathers the corresponding discretization coefficients. Similar to the standard approach, see e.g. Bul08, the desired tridiagonal Hamiltonian can be obtained by equating it to the Hamiltonian in the "star basis",

$$
H_{\text {band }}=\sum_{n} A_{n} \boldsymbol{\Gamma}_{n}^{+} A_{n}+B_{n} \boldsymbol{\Gamma}_{n}^{-} B_{n} \stackrel{!}{=} \sum_{n} F_{n}^{\dagger} \varepsilon_{n} F_{n}+F_{n}^{\dagger} \mathbf{t}_{n} F_{n+1}+F_{n+1}^{\dagger} \mathbf{t}_{n}^{T} F_{n}
$$

The spinors $F_{n}=\left(f_{n, \uparrow}, f_{n, \downarrow}^{\dagger}\right)^{T}$ correspond to the new tridiagonal chain, which is usually referred to as Wilson chain. From the above equality, a set of recursion relations for the new coefficients on the Wilson chain can be derived. In the case of a superconducting bath, the procedure is similar to the standard procedure, but completely formulated in the Nambu formalism. The detailed derivation is relegated to appendix B.

\subsubsection{Iterative Diagonalization}

The semi-infinite chain Hamiltonian on the righthand side of Eq. (2.29) allows to apply an iterative RG procedure since it can be defined as the limit of a series of finite-size Hamiltonians,

$$
\begin{aligned}
H & =\lim _{N \rightarrow \infty} \Lambda^{-N / 2} H_{N} \\
H_{N} & =\Lambda^{N / 2}\left(H_{\mathrm{imp}}+H_{\mathrm{hyb}}+\sum_{n=0}^{N} F_{n}^{\dagger} \varepsilon_{n} F_{n}+F_{n}^{\dagger} \mathbf{t}_{n} F_{n+1}+F_{n+1}^{\dagger} \mathbf{t}_{n}^{T} F_{n}\right)
\end{aligned}
$$


The factor $\Lambda^{ \pm N / 2}$ rescales the energies and cancels the energy dependence of the lowest lying hopping process $\mathbf{t}_{N}$, which is the hopping between the last two chain sites $N$ and $N+1$. Here the RG character becomes apparent: A single NRG iteration is defined recursively as

$$
H_{N+1}=R\left[H_{N}\right],
$$

which itself cannot have fixed points. Instead, $R^{2}: H_{N} \rightarrow H_{N+2}$ defines a RG flow with possible fixed points [Wil75, Km80a]. The RG transformation of the Hamiltonian, $R: H(\vec{K}) \rightarrow H\left(\vec{K}^{\prime}\right)$, successively absorbs high energy excitations into new renormalized coupling constants $\vec{K}^{\prime}$, which can, however, not be obtained from the $H_{N}$ in the NRG iterations. Nevertheless, $H_{N}$ and the RG flow are characterized by the corresponding many-particle energies $E_{N}$. The iterative diagonalization, in which these energies are obtained, proceeds as follows.

One starts with the set up of the Fock space of the impurity site $(N=-1)$, which is decoupled from the rest of the chain. It can be easily diagonalized as the number of eigenstates $|\phi\rangle_{-1}$ is small. In the case of a single channel model, for example, there are only four states of the impurity. In the next step, a basis for the system including the first chain site $(N=0)$ is simply obtained as the tensor product of the impurity Fock space with the Fock space of the first chain site. This new two-site cluster is then diagonalized again and the eigenstates are used to set up a basis for the enlarged system including the next site, and so on. For $N>0$ this scheme generalizes to

$$
|r ; s\rangle_{N+1}=|r\rangle_{N} \otimes|s[N+1]\rangle
$$

where a state of the enlarged chain $N+1$ is build from the tensor product of the eigenstates $|r\rangle_{N}$ of the smaller chain and the eigenstates $|s[N+1]\rangle$ of the single added site. With these eigenstates, the matrix elements of the new Hamiltonian $H_{N+1}$ can be calculated. Its diagonalization yields the new eigenvalues $E_{N+1}$ and eigenstates. The problem of this procedure is an exponential growth of number of states in the Fock space. For a complete diagonalization one is typically limited to a few sites. Thus, one has to reduce the number of states and truncate the Fock space. It turns out, that simply retaining the $N_{s}$ lowest lying sates after the diagonalization of $H_{N+1}$ works quite well Bulla2008. The maximum iteration number in the NRG has to be taken sufficiently high in order to reach the low-temperature fixed point.

\subsubsection{Calculation of Static and Dynamic Properties}

In the superconducting case, both static and dynamic expectation values of off-diagonal quantities have to be calculated for the impurity in addition to the diagonal ones. Examples 
are the local anomalous expectation value $\Phi=\left\langle d_{\uparrow} d_{\downarrow}\right\rangle$ or the off-diagonal Green's function $\left\langle\left\langle d_{\uparrow} ; d_{\downarrow}\right\rangle_{\omega}\right.$. The standard procedure is straightforward and is detailed in the literature [Bul08. For completeness, it is only briefly sketched here. Static expectation values can be calculated by setting up a matrix before the diagonalization procedure. The matrix is updated in each iteration step $N$ to the current basis. By means of the corresponding energy values $E_{i}^{(N)}$ and Boltzmann weights $\beta$, the static thermodynamic expectation values of a general operator $\hat{O}^{(N)}$ for the chain of size $N$ can be calculated as

$$
\langle\hat{O}\rangle^{(N)}=\frac{1}{Z^{(N)}} \sum_{i} \mathrm{e}^{-\beta E_{i}^{(N)}}{ }_{N}\langle i|\hat{O}| i\rangle_{N}
$$

where $Z^{(n)}$ is the respective partition function and $i$ labels the quantum numbers. Although the Fock space is truncated, this is often already a good approximation for $\langle\hat{O}\rangle\left(T_{N}\right)$, where the sum should rather run over a complete basis. The relevant contributions originate from a chain with the length, where the energy scale of the last site corresponds to the given temperature [Bul08]. However, a more advanced approach incorporating the complete basis set [And05, And06, Pet06, Wei07] is used for more precise calculations, especially at finite temperatures.

For the more intricate calculation of dynamic quantities, such as spectral functions, one can use the Lehmann representation - after the NRG calculation all necessary ingredients are basically available: eigenstates and -energies and the corresponding expectation values which can be used in

$$
\rho_{\hat{O}_{1} \hat{O}_{2}}(\omega, T)=\frac{1}{Z} \sum_{i, j} \delta\left(\omega+E_{i}-E_{j}\right)\left\langle i\left|\hat{O}_{1}\right| j\right\rangle\left\langle j\left|\hat{O}_{2}\right| i\right\rangle\left[\mathrm{e}^{-\beta E_{i}}+\mathrm{e}^{-\beta E_{j}}\right]
$$

with the partition function $Z=\sum_{i} \exp \left(-\beta E_{i}\right)$. Now the sum has to run over the complete basis: Neither the basis of the last iteration, nor a sum over all iterations of the chain is appropriate without further approximations. One can again overcome these problems by introducing a complete Fock-space basis [And05, And06, Pet06, Wei07].

\subsubsection{Calculation of the Self-Energy}

Another important dynamical quantity, which needs to be calculated for DMFT, is the self-energy. Although the self-energy can in principle be determined from Eq. (2.17), it turned out that it is more reliable to calculate the self-energy directly in NRG by the ratio of two correlation functions of the Anderson impurity model [Bul98]. For superconducting systems, this method needs to be generalized to the matrix structure of the Nambu formalism [Bau09]. One starts with the equation of motion for two fermionic operators $a$ 
and $b$,

$$
z\langle\langle a ; b\rangle\rangle_{z}+\left\langle\left\langle[H, a]_{-} ; b\right\rangle\right\rangle_{z}=\left\langle[a, b]_{+}\right\rangle
$$

Applying it to each component of the Green's function matrix of the impurity,

$$
\mathbf{G}_{d}(z)=\left\langle\left\langle D ; D^{\dagger}\right\rangle\right\rangle_{z}=\left(\begin{array}{ll}
\left\langle\left\langle d_{\uparrow} ; d_{\uparrow}^{\dagger}\right\rangle\right\rangle_{z} & \left\langle\left\langle d_{\uparrow} ; d_{\downarrow}\right\rangle\right\rangle_{z} \\
\left\langle\left\langle d_{\downarrow}^{\dagger} ; d_{\uparrow}^{\dagger}\right\rangle\right\rangle_{z} & \left\langle\left\langle d_{\downarrow}^{\dagger} ; d_{\downarrow}\right\rangle\right\rangle_{z}
\end{array}\right),
$$

one obtains a self-energy matrix

$$
\boldsymbol{\Sigma}(z)=\left\langle\left\langle[H, D]_{-} ; D\right\rangle\right\rangle_{z} \cdot \mathbf{G}(z)^{-1}
$$

where the matrix $\left\langle\left\langle[H, D]_{-} ; D\right\rangle\right\rangle_{z}$ incorporates higher Green's functions, depending on the exact form of the local interaction on the impurity. The symmetry relations of the normal and higher Green's functions [Bau09] ensure the symmetry of the self-energy matrix given in Eq. 2.21, so that the computational effort in NRG can be reduced. 


\section{The Kondo-Lattice Model}

In this chapter two of the most important models for Heavy-Fermion systems are introduced: the periodic Anderson model (PAM) and its effective low-energy simplification in a certain parameter regime, the Kondo-lattice model (KLM). Both belong to the most studied manyparticle models in solid state theory. They qualitatively describe some of the rich physics observed in HF systems, originating from the interplay of itinerant conduction electron in $s^{-}, p$-, or $d$ - bands with the rather localized $f$-electrons. The orbitally non-degenerate PAM accounts for this interplay in an idealized way such that the non-degenerate $f$-orbitals are subject to an intrasite Coulomb interaction $U$ and a hybridization $V$ with a single non-interacting conduction band. In the so-called Kondo-regime, the effective physics of the PAM can be captured by the KLM; it describes singly occupied $f$-orbitals as a quantum-mechanical spin- $\frac{1}{2}$ which is coupled via an antiferromagnetic exchange interaction $J$ to the local spin density of itinerant conduction electrons.

First, the PAM is introduced and afterwards its connection to the KLM is discussed. Some insight to the physical properties of the KLM can be gained in a simple mean-field description (section 3.1.2 and a more elaborate analysis within the DMFT+NRG approach (section 3.2). In real systems additional interactions are present among the electronic subsystem, such as a Coulomb interaction or a coupling to a local Holstein phonon mode. These extensions of the KLM and their influence on the paramagnetic properties are discussed at the end of the chapter.

\subsection{Periodic Anderson and Kondo Lattice Model}

Heavy-fermion systems are typically described with the periodic Anderson model And61, Ric85, Bla87, Gre06]. It captures the essential physics of non-degenerate $f$-orbitals coupled 
to a band of itinerant conduction electrons (Fig. 3.1) and reads

$$
H_{\mathrm{PAM}}=-\sum_{\langle i j\rangle \sigma} t_{i j} c_{i \sigma}^{\dagger} c_{j \sigma}+\varepsilon_{f} \sum_{i} n_{i \sigma}^{f}+\sum_{i \mathbf{k} \sigma} V_{\mathbf{k}}\left(c_{\mathbf{k} \sigma}^{\dagger} f_{i \sigma}+f_{i \sigma}^{\dagger} c_{\mathbf{k} \sigma}\right)+U \sum_{i} n_{i \uparrow}^{f} n_{i \downarrow}^{f}
$$

with $n_{\sigma}^{f}=f_{\sigma}^{\dagger} f_{\sigma}$. The first term describes the kinetic energy of the conduction electrons due to hopping processes with a tunneling matrix element $t_{i j}, \varepsilon_{f}$ denotes the atomic energy level of the $f$-orbital, $V$ is the $c$ - $f$-hybridization matrix element and $U$ denotes the local Coulomb repulsion among $f$-electrons.

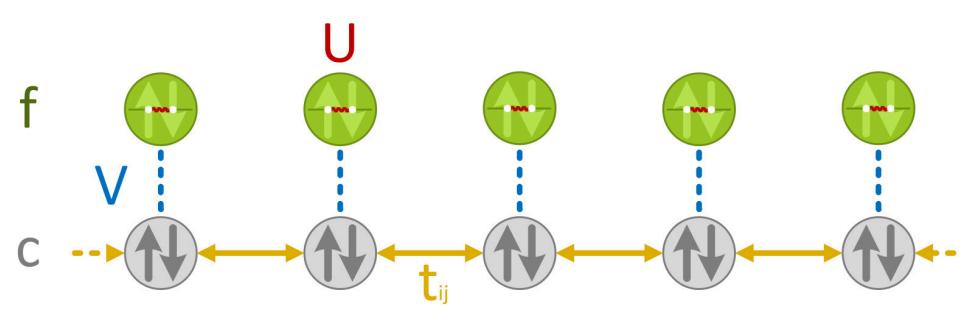

Figure 3.1: Sketch of the periodic Anderson model. Conduction (c) electrons are mobile due to the hopping $t_{i j}$. Each site of the conduction band hybridizes with a local $f$-orbital with an energy level $\varepsilon_{f}$. The strength of hybridization is given by $V$ and two $f$ electrons on the same site cost an energy $U$ due to Coulomb repulsion.

In the so-called Kondo regime relevant to HF systems the formation of a singly occupied $f$-level is favored and charge fluctuations are minimized. To generate such a situation, the singly occupied $f$-state $f^{1}$ has to lie lower in energy than the chemical potential, $\varepsilon_{f}<\mu$. On the other hand, it is necessary that the doubly occupied state $f^{2}$ lies above the chemical potential such that $\varepsilon_{f}+U>\mu$. In addition, the difference to $\mu$ should be rather large, i.e. $U \gg V$ in order to suppress charge fluctuations. In the latter case it is only the residual spin- $\frac{1}{2}$ degree of freedom which can interact with the conduction sea due to virtual charge fluctuations Col13

$$
e_{\uparrow}+f_{\downarrow}^{1} \leftrightarrow f^{2} \leftrightarrow e_{\downarrow}+f_{\uparrow}^{1} \quad \text { and } \quad e_{\uparrow}+f_{\downarrow}^{1} \leftrightarrow e_{\uparrow}+e_{\downarrow} \leftrightarrow e_{\downarrow}+f_{\uparrow}^{1}
$$

where $e_{\sigma}$ denotes an electronic state with spin $\sigma$ in the conduction band. These processes have the energies $\Delta E \propto U+\varepsilon_{f}$ in the first case and $\Delta E \propto-\varepsilon_{f}$ in the second case. This effective spin exchange happens in the singlet channel $S=0$.

Schrieffer and Wolff had shown that the PAM maps to a much simpler model in the so-called Kondo limit [Sch66]. The simple Kondo limit may be thought of either $V \rightarrow 0$ or $\left|\varepsilon_{f}\right|, \varepsilon_{f}+U \rightarrow \infty$ and thus corresponds to the weak-coupling regime of the KLM, $J \ll W$. The Schrieffer-Wolff transformation results in eliminating the hybridization term in first 
order, and to second-order in $V$ an effective exchange term with a coupling strength

$$
J=V^{2}\left(-\frac{1}{\varepsilon_{f}}+\frac{1}{\epsilon_{f}+U}\right)
$$

results. The full effective Hamiltonian reads

$$
H_{\mathrm{KLM}}=-t \sum_{\langle i j\rangle \sigma} c_{i \sigma}^{\dagger} c_{j \sigma}+\frac{J}{2} \sum_{i, \alpha \beta} \vec{S}_{i} \cdot\left(c_{i \alpha}^{\dagger} \boldsymbol{\sigma}_{\alpha \beta} c_{i \beta}\right)
$$

which is called the Kondo Lattice Model (KLM), see (Fig. 3.2..$\vec{S}_{i}$ represents the residual $f$-spin- $\frac{1}{2}$ and $\vec{s}_{i}=c_{i \alpha}^{\dagger} \boldsymbol{\sigma}_{\alpha \beta} c_{i \beta} / 2$ is the local spin density of conduction band electrons. ${ }^{1}$

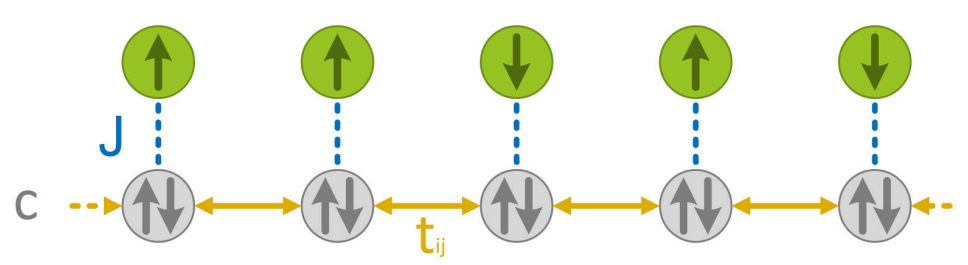

Figure 3.2: Sketch of the Kondo-lattice model. At each site of the conduction band (c) the local spin density couples via an antiferromagnetic exchange interaction $J$ to a local spin- $\frac{1}{2}$ degree of freedom.

In HF systems the Kondo coupling $J$ between $\vec{S}$ and $\vec{s}$ is always positive and thus acts as an antiferromagnetic exchange interaction. For the case of half filling of the $f$ band one has $\varepsilon_{f}=-U / 2$ and obtains $J=4 V^{2} / U$. Then, an exact mapping from the PAM to the KLM is possible for any finite value of $J$ in an extended Kondo limit [Sin02]: While keeping $V^{2} / U$ constant, one takes $U \rightarrow \infty$ and $V \rightarrow \infty$. From the numerical perspective, both models become effectively equivalent already for $U>5 W$ and one can actually extract low energy properties of the PAM such as the quasiparticle residue from the much simpler KLM [Don12].

\subsubsection{Energy Scales and the Doniach Diagram}

As the KLM results from a strongly correlated system, namely the PAM with a large Coulomb repulsion among felectrons, it also constitutes a model of strongly correlated

1 In the given form it is possible to account for higher spins $S=1, \frac{3}{2}, 2, \ldots$ also. For example, the $S=1$ Kondo-lattice model is relevant to HF compounds containing Uranium. As a single conduction band cannot fully screen the $f$-spins for $S>\frac{1}{2}$, the model is then referred to as the underscreened KLM in the literature. 
electrons - even in the weak-coupling regime. Depending on the strength of $J$, the effective interactions in the KLM at low temperatures can be classified by two different regimes. In the case of weak coupling $J \ll W$ the Ruderman-Kittel-Kasuya-Yosida (RKKY) [Rud54, Kas56, Yos57] interaction dominates the physics: It is a non-local indirect exchange interaction between the localized $f$-spins and is generated in second order perturbation theory of the KLM. In the opposite strong coupling limit, $J \gg W$, the system is governed by local Kondo-singlet formation which competes with the RKKY interaction.

\section{Weak Coupling: The RKKY Interaction}

The sharp Fermi surface in metals and a spin-dependent scattering potential lead to Friedel oscillations [Fri52] in the spin-density around a magnetic moment $\vec{S}_{1}$ immersed in the electron sea. The surrounding of a second magnetic moment $\vec{S}_{2}$ nearby is therefore locally spin-polarized and $\vec{S}_{2}$ will lower its energy by aligning either ferromagnetically or antiferromagnetically with it. The sign of the magnetic interaction depends on the spatial distance $r$ between magnetic moments and oscillates as function thereof. In HF systems the RKKY interaction is typically antiferromagnetic and the characteristic dependence on $r$ and the Kondo coupling $J$ is given by

$$
J_{\mathrm{RKKY}} \propto J^{2} \rho_{0} \frac{\cos \left(k_{F} r\right)}{\left(k_{F} r\right)^{\alpha}}
$$

with a positive dimension-dependent number $\alpha$ and an additional exponential spatial decay not included here. Strictly speaking, this form is valid only in the limit $J \rightarrow 0$. It is far from clear whether the RKKY picture extends to the limit of infinite dimensions in the case of DMFT. However, it has been shown that in this limit the RKKY interaction depends on the momentum derivative of the local susceptibility via [MH89, Pet07]

$$
\left.J_{\mathrm{RKKY}} \propto J^{2} \frac{\mathrm{d}}{\mathrm{d} x} \chi^{z z}(x)\right|_{x=0} \text { with } \quad x=\lim _{d \rightarrow \infty} \frac{1}{d} \sum_{i=1}^{d} \cos \left(q_{i} a\right)
$$

for a hypercubic lattice with lattice constant $a$ and momenta $q_{i}$. The behavior of $J_{\text {RKKY }}$ in the limit $d \rightarrow \infty$ is shown to be filling-dependent [Pet07]. For $n \leq 0.45$ the effective interaction is ferromagnetic, while there is a sign change for $n>0.45$ to an antiferromagnetic interaction. In this thesis calculations are only shown for $n>0.5$, hence a weak Kondo interaction typically yields an antiferromagnetic intersite coupling. 


\section{Strong Coupling: Kondo-Singlet Formation}

For a strong antiferromagnetic coupling it is evident that the conduction band electrons tend to build a many-particle Kondo-singlet state with the $f$-spins. The formation of Kondo singlets is associated with the single-ion Kondo temperature $T_{K}$. In the dense lattice of local moments the situation is qualitatively different to the case of diluted magnetic impurities: While incoherent Kondo screening sets in at $T_{K}$ already, the spin scattering develops coherence below a corresponding temperature $T_{0}$ (usually $T_{0}<T_{K}$ ) and the resistance of the system drops down drastically, as in the case of $\mathrm{Ce}_{\mathrm{x}} \mathrm{La}_{1-\mathrm{x}} \mathrm{Cu}_{6}$ for a doping $x \rightarrow 1$ [Onu87]. The coherence below $T_{0}$ also indicates the Fermi-liquid regime of the renormalized heavy composite quasiparticles with a heavy mass. A special case occurs for half filling, $n_{c}=1$ : On average every $c$-electron is bound into a singlet with an $f$-spin $(1 / 2)$ and thus the system is insulating.

\section{Bridging the Regimes: The Doniach Diagram}

A qualitative understanding of the transition regime between weak and strong coupling limits of the Kondo lattice was put forward by Doniach [Don77]. In the so-called Doniach diagram (Fig. 3.3) a second order phase transition between the antiferromagnetic to a Kondo-spin compensated groundstate was suggested. It results from the crossing of two relevant energy scales in Kondo lattice systems, the aforementioned Kondo temperature or Kondo-singlet binding energy $T_{K} \propto \exp (-\alpha / J)$ and the RKKY interaction scale $E_{\mathrm{RKKY}} \propto J^{2} \rho_{0}$. As function of $J$ both scales cross at a critical coupling $J_{c}$, below which $T_{K}<E_{\mathrm{RKKY}}$ and the system orders antiferromagnetically at low temperatures, and above which the coherent Kondo screening dominates $\left(T_{K}>E_{\mathrm{RKKY}}\right)$. Both energy scales are proportional to the critical temperatures of the RKKY and heavy Fermi liquid phase, respectively. At zero temperature the regimes are divided by a quantum critical point.

Quantum critical fluctuations around QCPs also influence the physics at finite temperatures: Many HF systems show a peculiar non-Fermi liquid (NFL) behavior above a QCP. Often the QCPs are indeed hidden by a superconducting dome [Ste12a] as sketched in (Fig. 3.3. and thus the connection between quantum critical fluctuations and superconductivity is a matter of current research Onu13.

\subsubsection{Static Mean-Field Description of the KLM}

The picture of composite quasiparticles from hybridized bands is further elucidated in a mean-field treatment of the KLM. Moreover, the static mean-field description serves 


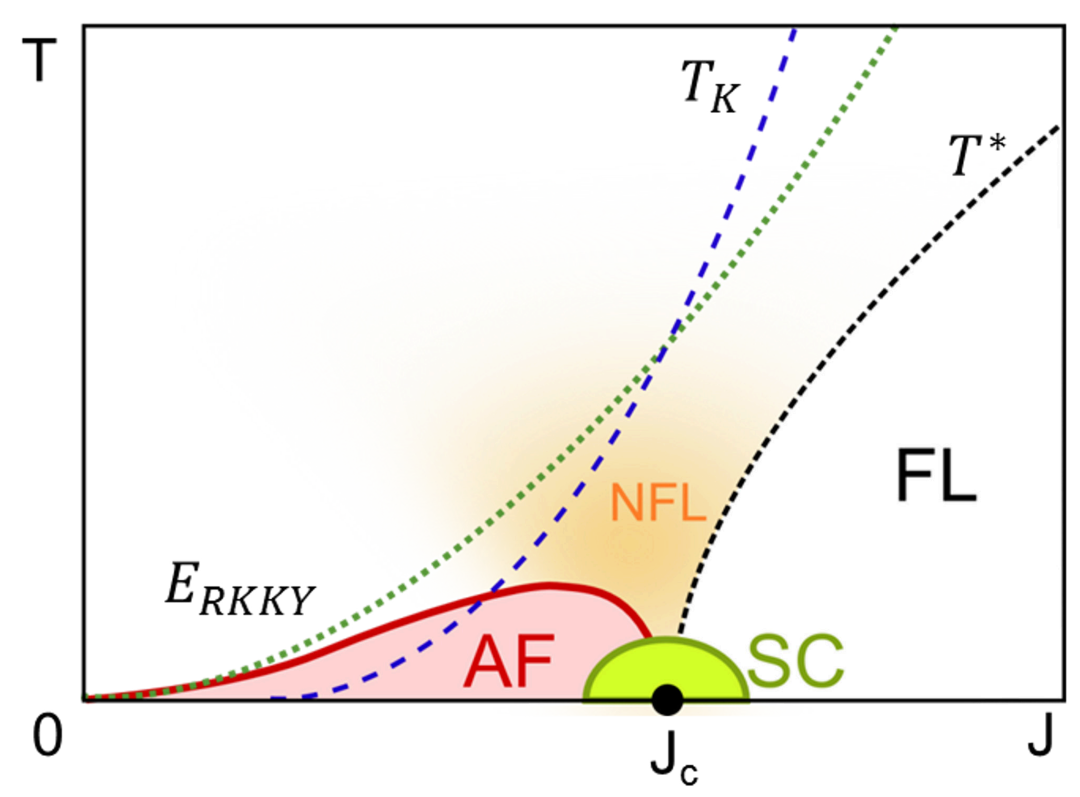

Figure 3.3: Schematic Doniach diagram as function of exchange coupling $J$ showing the competing energy scales $E_{\mathrm{RKKY}}$ (dotted green) and $T_{K}$ (dashed blue). At the point where both energy scales cross, a QCP exists at zero temperature. It divides the antiferromagnetically ordered (AF) phase from the Fermi-liquid (FL) regime. Quite often the QCP is hidden by a dome of superconducting (SC) order above which a non-Fermi-liquid (NFL) regime exists. $T^{*}$ denotes the coherence scale below which a $\mathrm{FL}$ is formed.

as a reference to which DMFT results can be compared to. As the derivation of the mean-field model for the Kondo lattice also includes some important physical insight, it will be outlined in the following.

The spin operators in the Kondo-lattice Hamiltonian in Eq. 3.2 can be rewritten in a fermionic representation

$$
\begin{aligned}
S_{i}^{z} & =\left(n_{i \uparrow}^{f}-n_{i \downarrow}^{f}\right) / 2 \\
S_{i}^{+} & =f_{i \uparrow}^{\dagger} f_{i \downarrow} \\
S_{i}^{+} & =f_{i \downarrow}^{\dagger} f_{i \uparrow}
\end{aligned}
$$

i.e., one uses auxiliary fermionic fields $f$. This mapping is exact only if one adds the constraint of single occupation of the $f$-levels at each site, $n_{i}^{f}=n_{i \uparrow}^{f}+n_{i \downarrow}^{f}=1$. Thus, the charge of the auxiliary fermions is conserved, $Q_{i}^{f}=1$, and this representation of the $f$-shell 
local moments has a local U(1) gauge symmetry. The KLM can then be written as

$$
H_{\mathrm{KLM}}=-t \sum_{\langle i j\rangle \sigma} c_{i \sigma}^{\dagger} c_{j \sigma}+\frac{J}{4} \sum_{i, \alpha \beta}\left(f_{i \alpha}^{\dagger} \boldsymbol{\tau}_{\alpha \beta} f_{i \beta}\right) \cdot\left(c_{i \alpha}^{\dagger} \boldsymbol{\tau}_{\alpha \beta} c_{i \beta}\right)
$$

that is, the Kondo interaction now includes four-fermion terms. These can be decoupled so as to obtain a mean-field theory for the KLM. In the standard approach, a functionalintegral representation of the KLM is introduced and a Hubbard-Stratonovich transform in the particle-hole channel is carried out, whereby additional bosonic fields $V_{i}, V_{i}^{*}$ are introduced [Rea83]. Thereafter the interaction term reads ${ }^{1}$

$$
H_{V}=\sum_{i \sigma}\left[V_{i \sigma}\left(c_{i \sigma}^{\dagger} f_{i \sigma}\right)+\left(f_{i \sigma}^{\dagger} c_{i \sigma}\right) V_{i \sigma}^{*}+2 V V^{*} J^{-1}\right]
$$

In addition, the constraint $Q_{i}^{f}=1$ has to be dynamically enforced by a time-dependent bosonic field ${ }^{2} \lambda_{i}$ that couples to $\left(n_{i}^{f}-Q_{i}^{f}\right)$. The full Lagrangian $\mathcal{L}(\tau)$ in the functional integral for the partition sum

$$
\mathcal{Z}_{\mathrm{KLM}}=\int \mathcal{D}\left(c, c^{\dagger}\right) \mathcal{D}\left(f, f^{\dagger}\right) \mathcal{D}\left(V, V^{*}\right) \mathcal{D} \lambda \exp \left(-\int_{0}^{\beta} \mathrm{d} \tau \mathcal{L}(\tau)\right)
$$

then reads

$$
\mathcal{L}(\tau)=\sum_{i \sigma}\left[f_{i \sigma}^{\dagger}\left(\partial_{\tau}+\lambda\right) f_{i \sigma}+c_{-\sigma}^{\dagger}\left(\partial_{\tau}+\varepsilon_{k}\right) c_{i \sigma}-\lambda\right]+H_{V}
$$

This Lagrangian was shown to be invariant under a certain gauge transformation, the Read-Newns transformation [Rea83]. The gauge phase can be absorbed in the field $\lambda$ and then $V$ becomes real. By this Anderson-Higgs-type mechanism the $f$-degrees of freedom are endowed with a physical charge and can be regarded as electrons. This process justifies the standard picture of two hybridized electronic bands once the heavy Fermi liquid has formed.

The above derivation may be interpreted as a sort of reverse Schrieffer-Wolff transformation of the KLM to the periodic Anderson model, though without a Coulomb repulsion among $f$-electrons. The philosophy is, however, quite different. The KLM serves as an effective model for the PAM in the Kondo-limit, with an effective spin- $\frac{1}{2}$ per site originating from electrons in $f$-orbitals. From this perspective, the picture of additional electronic states, i.e., composite heavy fermions, appears to emerge naturally from the hybridization with $f$-electrons. However, the spins in the KLM could also be of nuclear origin. In this case,

1 Note that the $V_{i}$ are full fluctuating bosonic fields for the time being.

2 The $\lambda$-fields are actually dynamic Lagrange multipliers, see e.g. Col13. 
the mean-field decoupling implies the generation of additional electronic states near the Fermi energy from nuclear spins once the lattice Kondo effect takes place. In the single-ion Kondo model this leads to a Kondo resonance at the Fermi energy, while in the dense Kondo lattice the new states form the coherent heavy quasiparticle band.

In the following the hybridized-band picture is elucidated in a bit more detail. The decoupling introduced above motivates a saddle-point approximation, that is, the fluctuating fields $V(\tau)$ and $\lambda(\tau)$ are replaced by their static values at the saddle-point of the partition function. The resulting mean-field Hamiltonian for the KLM reads

$$
H_{\mathrm{KLM}}^{\mathrm{MF}}=\sum_{\mathbf{k} \sigma}\left(\varepsilon_{\mathbf{k}}-\mu\right) n_{\mathbf{k} \sigma}^{c}+\lambda \sum_{i} n_{i \sigma}^{f}+V \sum_{i \sigma}\left(c_{i \sigma}^{\dagger} f_{i \sigma}+f_{i \sigma}^{\dagger} c_{i \sigma}\right)+N_{s} \frac{|V|^{2}}{J} .
$$

Since $\lambda$ effectively constitutes an on-site energy, it is renamed by $\varepsilon_{f}$. Both $V$ and $\varepsilon_{f}$ in principle have to be determined self-consistently. After a Fourier transform the Hamiltonian can be rewritten in a matrix form $(\mu=0$ in the following)

$$
H_{\mathrm{KLM}}^{\mathrm{MF}}=\sum_{\mathbf{k} \sigma}\left(\begin{array}{cc}
c_{\mathbf{k} \sigma}^{\dagger} & f_{\mathbf{k} \sigma}^{\dagger}
\end{array}\right)\left(\begin{array}{cc}
\varepsilon_{\mathbf{k} \sigma} & V \\
V & \varepsilon_{f}
\end{array}\right)\left(\begin{array}{c}
c_{\mathbf{k} \sigma} \\
f_{\mathbf{k} \sigma}
\end{array}\right)
$$

and the quasiparticle energies can be easily obtained by diagonalizing this matrix. For a finite hybridization $V$ the resulting energies split in two branches, also referred to as hybridized bandstructure of the KLM:

$$
E_{\mathbf{k}}^{ \pm}=\frac{\varepsilon_{f}+\varepsilon_{\mathbf{k}} \pm \sqrt{\left(\varepsilon_{\mathbf{k}}-\varepsilon_{f}\right)^{2}+4 V^{2}}}{2}
$$

In (Fig. 3.4 the typical qualitative shape of quasiparticle bands in the paramagnetic KLM according to Eq. 3.7) is shown. Essential quantities are a direct gap of width $2 V_{\text {eff }}$, an indirect hybridization gap $\Delta_{g}$ and the renormalized chemical potential $\varepsilon_{f}$ of the $f$-level. The eigenvectors of the mean-field Hamiltonian Eq. (3.6) are mixtures of $c$ - and $f$-degrees of freedom, the weight of which is indicated in (Fig. 3.4) by color: The strongly dispersive parts of the upper and lower band correspond to the conduction $c$-electrons (blue) while the flat parts belong to $f$-electrons (green). Although the $f$-level itself is non-dispersive in its origin, it gains a slight dispersion by hybridization. These flat and almost dispersionless segments of the quasiparticle bands close to $E_{\mathbf{k}} \approx \varepsilon_{f}$ correspond to the quasiparticles with a strongly renormalized mass, i.e., heavy fermions.

In the hybridized-band picture, the insertion of electronic states in the conduction band can be understood by noting that the intersection of the lower band with the Fermi energy between the $\Gamma$ - and $M$-point shifts towards $\mathbf{k}=(\pi, \pi)$. It thus yields a large Fermi surface. A rigorous proof of this fact is far from trivial. While Luttinger's theorem [Lut60] 

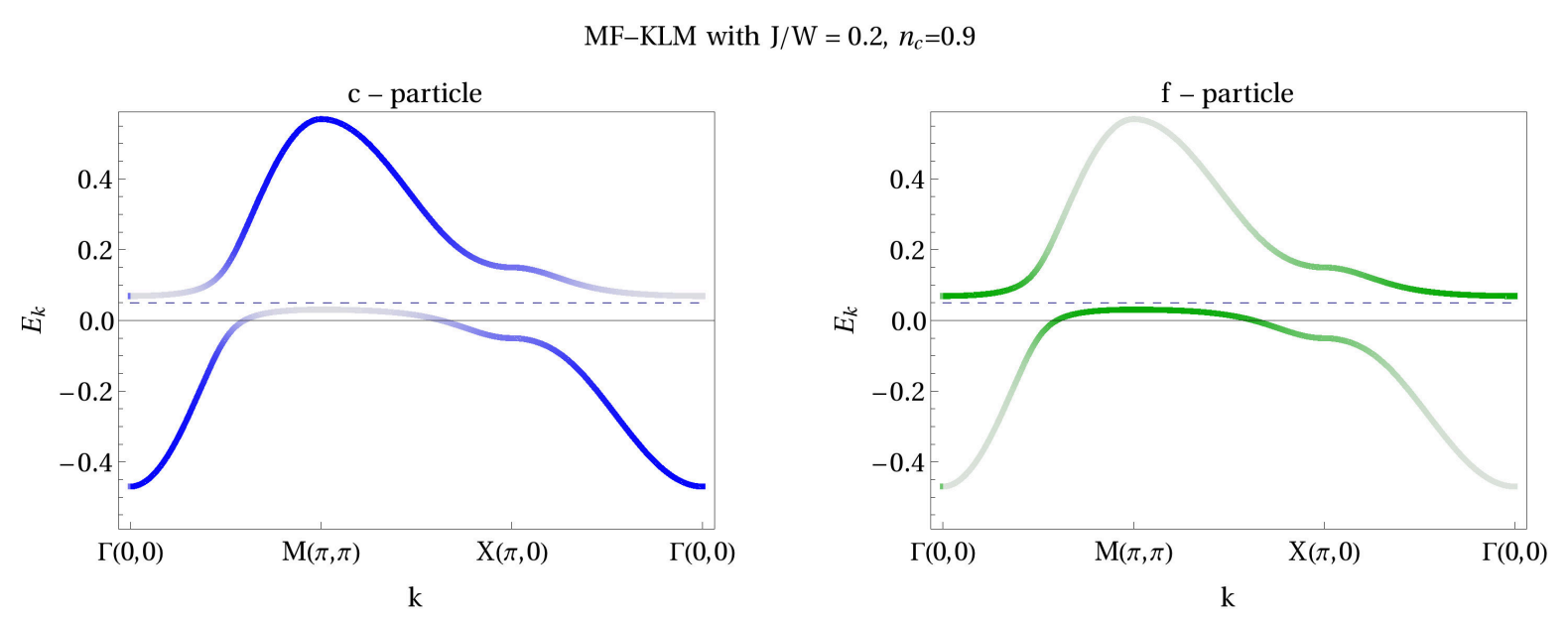

Figure 3.4: Visualization of hybridization in a typical renormalized quasiparticle bandstructure of the KLM in the mean-field picture. Left: Weight of $c$-states. Right: Weight of $f$-states. The dashed line indicates the position of $\varepsilon_{f}$.

states that the Fermi volume of a metal is proportional to the fermionic particle density, $\mathcal{V}_{f} \propto n_{c}+n_{f}$, which certainly applies to the PAM with a hybridization [Lan66], spin degrees of freedom are not included. The localized spins in the Kondo lattice do, however, contribute to the Fermi surface provided that Kondo screening is intact [Mar82]. Another version of Luttinger's theorem [Osh00] based on topological arguments proves this fact. A large hole-like Fermi surface is thus always present in the paramagnetic phase of the KLM with a finite antiferromagnetic Kondo coupling.

However, in the antiferromagnetic phase of the KLM a small Fermi surface is possible. For weak coupling, the RKKY interaction prevails such that the local moments are effectively decoupled from the conduction band electrons. Thereby, a transition from a particle-like to a hole-like Fermi surface is induced upon increasing $J$. Approaching this transition from the strong coupling regime, it is often called "Kondo breakdown". From a more general perspective it constitutes an example for an orbital-selective Mott transition Voj10. It has been proposed, that an additional energy scale $E_{\text {loc }}^{*}$ in the Doniach diagram separates the two regimes Geg08. At zero temperature it marks a $(f-)$ moment-localization phase transition while at elevated temperature it is more to be understood as a crossover regime. To the left of $E_{\text {loc }}^{*}$ Kondo screening is incomplete and the local moments do not deliver a crucial contribution to the Fermi volume. On the right of the $E_{\mathrm{loc}}^{*}$ line the local moments delocalize and do contribute to a large Fermi volume. At $T=0$ the position of $E_{\text {loc }}^{*}$ determines the nature of the QCP: If $E_{\text {loc }}^{*}$ hits $E_{\mathrm{RKKY}}$ at zero temperature, i.e., the AF phase boundary falls together with the breakdown of the Kondo effect, the quantum-critical fluctuations do include both the quantum fluctuations of the magnetic order parameter and the ones of the setting-in or destruction of Kondo screening. This case is termed a 
local QCP. In the other case $E_{\mathrm{loc}}^{*}$ crosses $E_{\mathrm{RKKY}}$ and the quantum-critical fluctuations at the antiferromagnetic QCP are only the ones of the order parameter. The local moments are delocalized in the part of the AF phase close to the QCP and the magnetic groundstate of the system can be described as a spin-density-wave state. The QCP is thus called spin-density-wave-type QCP.

\subsection{Paramagnetic Properties of the Kondo-Lattice Model}

The static mean-field treatment of the KLM gives some first insight into the nature of heavy quasiparticles. As the underlying mechanism of heavy-fermion physics is, however, a dynamic quantum many-particle effect, it is necessary to include quantum fluctuations for a more accurate description. In this section results for the KLM obtained from DMFT in combination with NRG are presented, where it is accounted for local quantum fluctuations. At high temperatures and small to intermediate Kondo coupling the DMFT analysis of the KLM well describes the system as consisting of localized moments Ots09. This result fits well with the experimentally measured Curie-like susceptibility $\chi \propto T^{-1}$ at elevated temperatures. With decreasing temperature the local moments gradually disappear and get immersed to the sea of conduction electrons. Heavy quasiparticles result.

The heavy quasiparticles manifest in a large Fermi surface according to Luttingers theorem, i.e., the spin degrees of freedom are included in the Fermi volume. For a two-dimensional dispersion and zero temperature, this is shown for the KLM with $J / W=0.15$ in (Fig. 3.5).
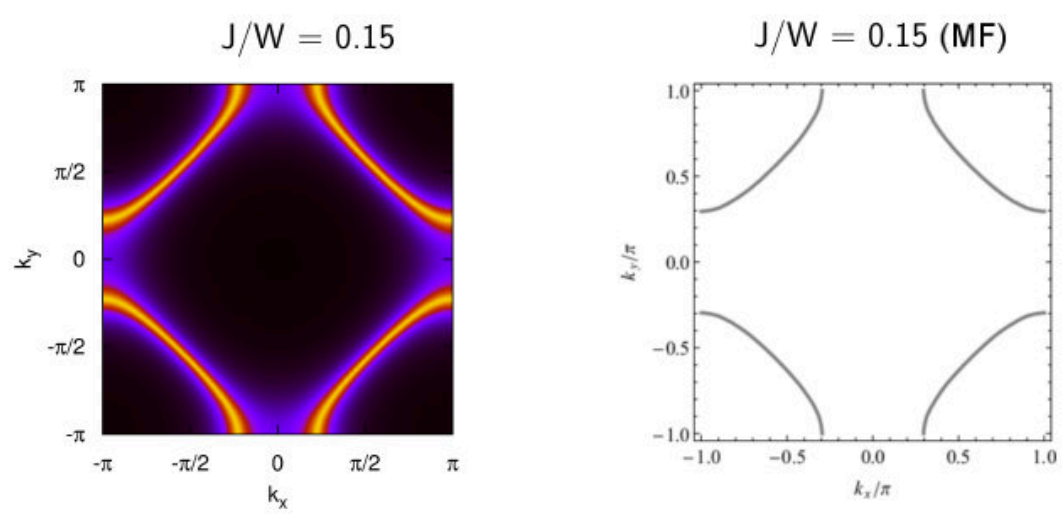

Figure 3.5: Left: Fermi surface of the KLM for $J / W=0.15$ at a fixed filling $n=0.9$ obtained by DMFT results. Right: Fermi surface for $J / W=V^{2} /\left(W \varepsilon_{f}\right)=0.15$ and $n=0.9$ in the mean-field description. 
The Fermi surfaces obtained by DMFT+NRG are qualitatively the same as those obtained in a simple mean-field treatment, as shown in (Fig. 3.5). However, the DMFT+NRG method allows access the low energy scale and dynamic quantities such as the density of states or self-energies more accurately. Some of these detailed Fermi-liquid properties of the KLM are discussed in the following.

\subsubsection{Fermi Liquid Properties}

In the normal paramagnetic state the KLM realizes a heavy Fermi liquid at low temperatures for $n \neq 1$ with a corresponding Fermi-liquid or coherence scale $T_{0}$. The coherence scale is typically much smaller than the single ion Kondo temperature $T_{K}$ in the metallic regime Pru00 and increases with $J$, that is, for larger $J$ the temperature up to which the Fermi liquid exists is also higher. In a local self-energy approximation, the Green function can be approximated near the Fermi surface as renormalized Fermi liquid

$$
G_{\mathbf{k}}(\omega) \approx \frac{Z}{\omega-Z\left[\varepsilon_{\mathbf{k}}-\mu+\Sigma(0)\right]} .
$$

The corresponding renormalization factor $Z$ or quasiparticle weight can be extracted from the local self energy by

$$
Z=\left(1-\left.\frac{\mathrm{dRe} \Sigma(\omega)}{\mathrm{d} \omega}\right|_{\omega=0}\right)^{-1}
$$

and yields a measure of the inverse effective mass $m^{*}$ of the quasiparticles, $Z=\mathrm{m} / \mathrm{m}^{*}$. The coherence scale $T_{0}$ can be easily extracted by $T_{0}=W \cdot Z, W$ being the bandwidth.

In (Fig. 3.6) the DOS of the KLM away from half filling $(n=0.9)$ is shown for three different Kondo couplings $J / W=0.2,0.3,0.4$. The pseudogap width reflects the coherence scale $T_{0}$ which grows with increasing $J$. The exact behavior of $T_{0}$ as function of $J$ is shown in the inset of (Fig. 3.6). For very small couplings $J \rightarrow 0$ the scale $T_{0}$ depends exponentially on both $J$ and the bare $c$-DOS, $T_{0} \propto \sqrt{J / W} \exp (-\alpha(n) \cdot W / J)$ [Bur00, Pru00]. The factor $\alpha(n)$ is only weakly $n$-dependent. For large Kondo couplings $J / W>0.5$ the $J$-dependence of $T_{0}$ significantly deviates from this Kondo-like form and tends to saturate in the limit of an infinite Kondo coupling.

Keeping $J$ fixed and sweeping the filling $n$ one observes a behavior of the DOS as in (Fig. 3.7). At half filling $n=1$ the system is an insulator in the sense that the DOS is fully gapped at the Fermi surface. This behavior stems from Kondo-singlet building at each lattice site. Away from half filling the DOS drops down right at the Fermi energy and the pseudgap persists as a residual feature. In the inset it is shown that the known dependence 


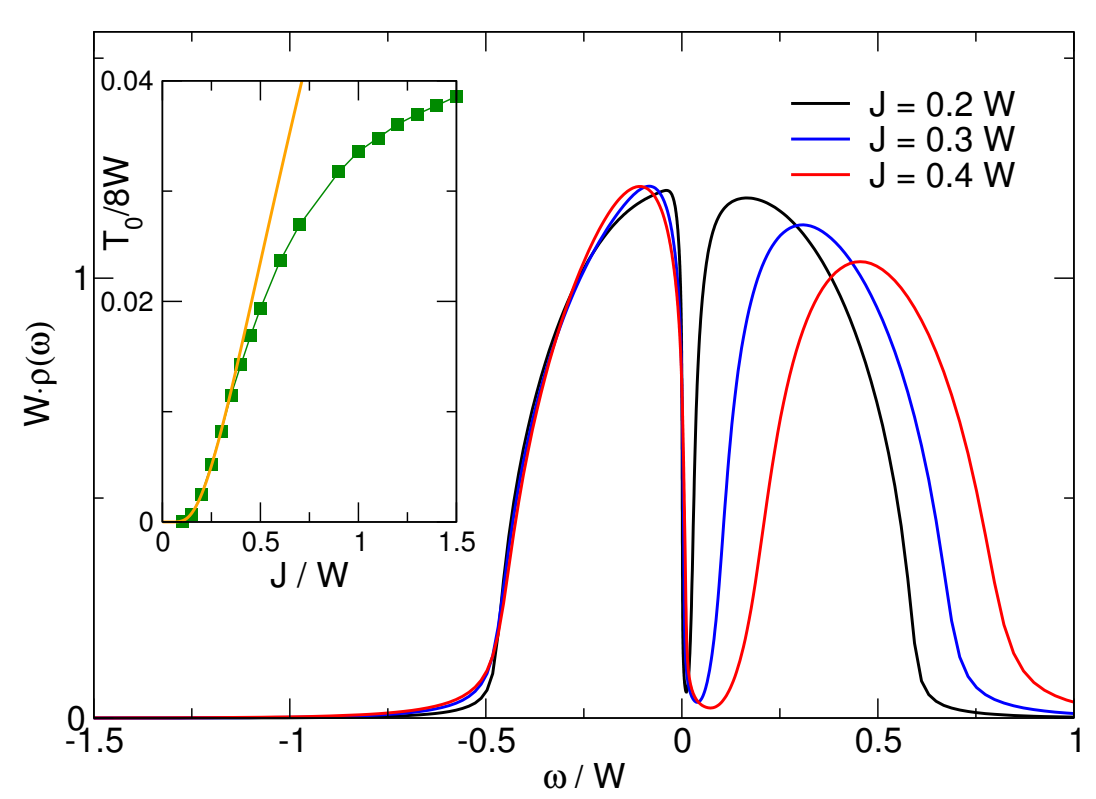

Figure 3.6: DOS of the KLM for $J / W=0.2,0.3,0.4$ and a filling $n=0.9$. The width of the pseudogap slightly above the Fermi energy reflects the coherence scale $T_{0}$ shown in the inset: At small couplings $T_{0}$ grows exponentially as function of $J$ (exponential fit function in orange).

$T_{0} \propto n \mathrm{e}^{c \cdot n}[\mathrm{Pru00}]$ is recovered. The lower the filling, the more $T_{0}$ is suppressed and the steeper the drop in the DOS at $\omega=0$. Close to quarter filling the large slope results in an excessive increase of the DOS at $\omega \leq 0$. The pseudogap is less marked for low fillings due to exhaustion of conduction electrons available for screening. In this region $n \lesssim 0.5$ the KLM typically orders ferromagnetically Pet07. 


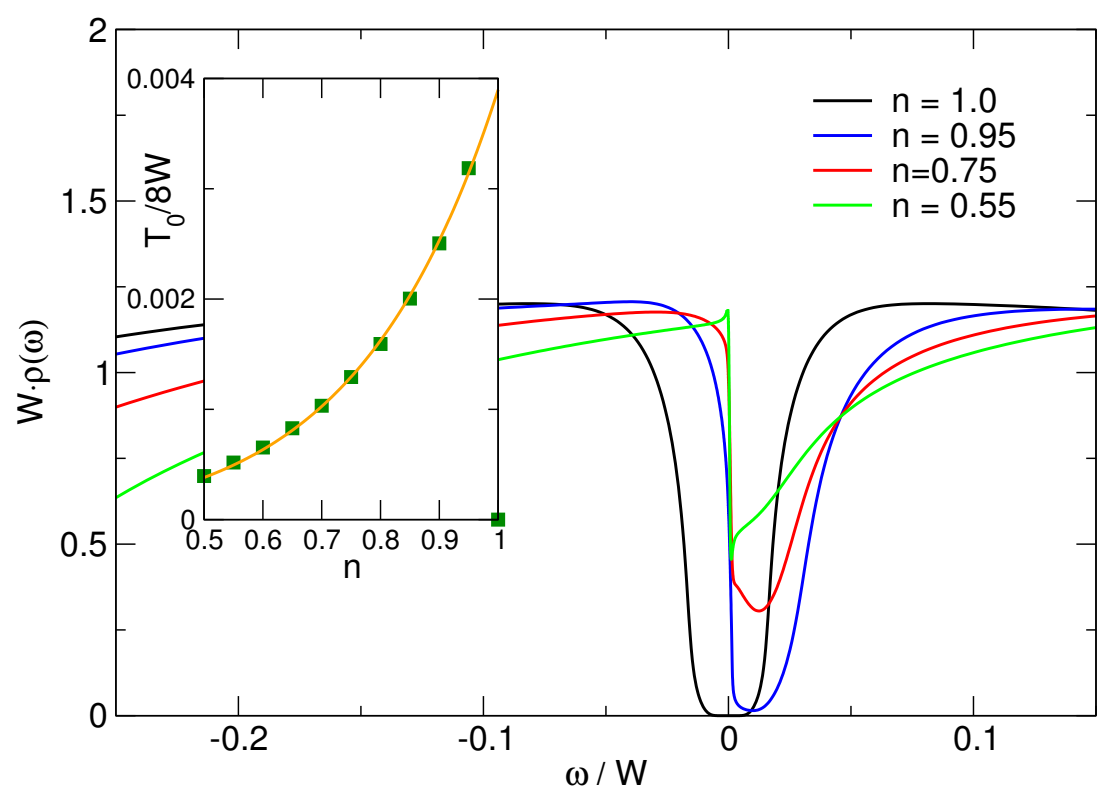

Figure 3.7: Close-up of the DOS for the KLM with $J / W=0.2$ at several fillings. Inset: Exponential behavior of the coherence scale $T_{0}$ as function of filling.

The situation very close to half filling deviates from the exponential form as function of $n$, cf. (Fig. 3.8). $T_{0}$ has a maximum around $n=0.99$ and drops down to zero at half filling since the system is a Kondo insulator there. The transformation of the gap at half filling to a pseudogap for finite doping is thus not completely abrupt but rather smooth in a very small region $0.99<n<1$.

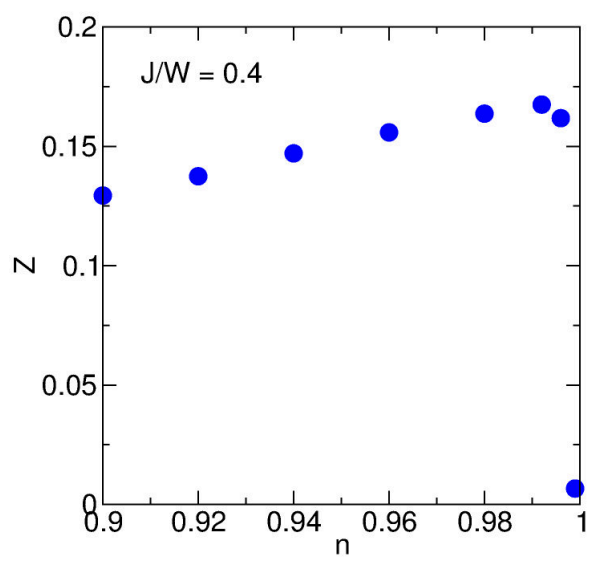

Figure 3.8: Filling dependence of the quasiparticle residue in the KLM with $J / W=0.4$. 


\subsubsection{Influence of Additional Interactions}

The KLM is most often studied without any additional interactions both for the sake of identifying only the most relevant physical aspects and applicability of many analytical and numerical methods. Nevertheless, in real systems there are many interactions adding up to the antiferromagnetic exchange coupling in HF systems. Here, we focus on the probably most important - two local interactions among conduction electrons: the Coulomb repulsion and the coupling to bosonic modes, effectively mediating a retarded interaction among electrons.

\section{Coulomb Repulsion}

The Coulomb repulsion among conduction band electrons is typically neglected in the KLM. It is nevertheless physically present and can well be of importance, especially in $3 d-4 f$ systems where the $3 d$ states can contribute in the conduction band, e.g., in Ce-compounds containing Co [Pat10]. Although this contribution may be weak, it can significantly change electronic properties. The correlated Kondo-lattice model, or sometimes called Kondo-Hubbard model, accounts for a local Coulomb repulsion in the conduction band:

$$
H_{\mathrm{CKL}}=H_{\mathrm{KLM}}+U \sum_{i} n_{i \uparrow} n_{i \downarrow}
$$

With respect to the HF physics, a conduction electron repulsion is expected to affect the competition between Kondo-singlet formation and magnetic order due to RKKY interactions. An on-site repulsion supports the tendency towards localization while the Kondo-singlet formation delocalizes the $f$ spins. For a two-dimensional KLM it was shown that for small $U$ and $J$ the on-site and inter-site spin correlations depend non-monotonically on $U$ [Sia12]. Even more important, the lattice coherence scale $T_{0}$ was reported to increase monotonically with $U$ and to reach a plateau in the large $U$ limit. As $T_{0}=W \cdot Z=W \cdot \frac{m}{m^{*}}$ this corresponds to a reduced effective mass. An increase of $T_{0}$ should thus bring along an increase of the pseudo-gap width in the DOS. In (Fig. 3.9) this behavior is confirmed. There the paramagnetic DOS of the KLM with an additional repulsion $U>0$ among the conduction band electrons is shown. For large $U$, the spectral weight in the DOS is shifted to a Hubbard peak around $\omega \approx U$. A small peak slightly above the upper limit of the pseudogap remains and marks the heavy quasiparticles which are retained in the renormalized band.

In fact, a local Coulomb repulsion of the order of bandwidth reinforces Fermi liquid behavior and leads to an increase of the renormalization factor of roughly $200 \%$, see inset of (Fig. 3.9). The stronger localization of the conduction band electrons results in a stronger interaction between local spin-density and Kondo spins; charge fluctuations on the conduction band 


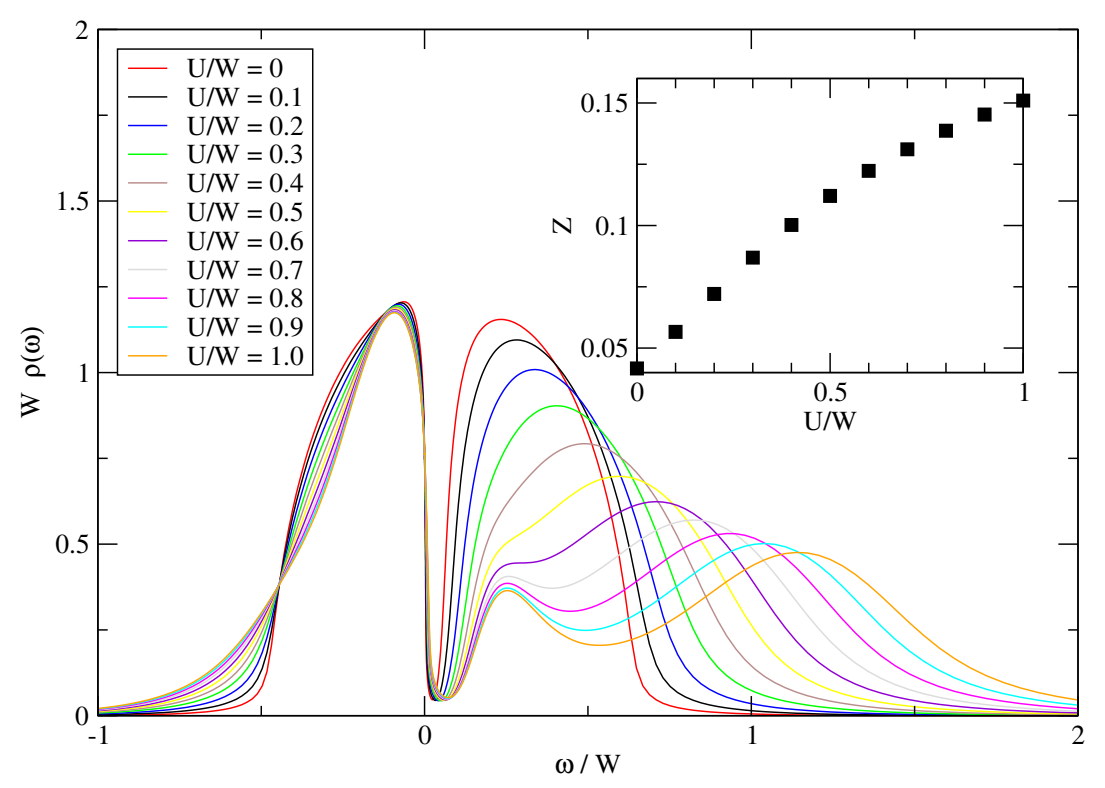

Figure 3.9: Development of the DOS of the correlated KLM as a function of Coulomb repulsion $U$ and with a fixed $J=0.2$. Inset: Corresponding renormalization factor as function of $U$.

site, which compete with local spin fluctuations, are increasingly suppressed with larger values of the repulsive $U$. From this point of view it is also clear, that an attractive interaction among conduction band electrons, should lead to the opposite case: an increase of charge fluctuations and thereby a suppression of spin fluctuations and the corresponding energy scale $T_{0}$.

\section{Holstein Phonons}

An attractive interaction among conduction electrons can be induced by a coupling of them to bosonic degrees of freedom, such as phonons, excitons or plasmons ${ }^{1}$. Phonons can induce such phenomena like superconductivity [Fro50], or the large volume collapse in Cerium which characterizes the structural $\gamma-\alpha$ phase transition [All82. The electronphonon coupling in real materials can be incorporated in the DMFT+NRG approach in an

1 For the sake of simplicity we will refer to the bosonic mode as "phonon" in the following. 
idealized way only [Geo96, Bul08]. The Holstein model [Hol59] captures such an electron phonon coupling for dispersionless Einstein modes with frequency $\Omega_{0}$, which are in addition harmonic and do not alter the hopping $t$ :

$$
H_{\text {Holstein }}=-\sum_{\langle i j\rangle, \sigma} t_{i j}\left(c_{i}^{\dagger} c_{j}+\text { h.c. }\right)+g \sum_{i} x_{i}\left(n_{i \uparrow}+n_{i \downarrow}\right)+\frac{M \Omega_{0}^{2}}{2} \sum_{i} x_{i}^{2}+\frac{1}{2 M} \sum_{i} p_{i}^{2}
$$

The phonon coordinate and its momentum at site $i$ are denoted as $x_{i}$ and $p_{i}$, respectively. The local oscillator displacement couples via $g$, the electron-phonon coupling constant, to the on-site electron density. In the following the oscillator mass is set to $M=1$, for simplicity. The spring constant is then $\kappa=\Omega_{0}^{2}$ and measures the energy per unit length squared being stored in the bosonic field. A more simple form of the Hamiltonian can be achieved by using the displacement operator $x_{i}=\left(b_{i}+b_{i}^{\dagger}\right) / \sqrt{2 \Omega_{0}}$ of the bosons, such that

$$
H_{\text {Holstein }}=-\sum_{\langle i j\rangle, \sigma} t_{i j}\left(c_{i}^{\dagger} c_{j}+\text { h.c. }\right)+g \sum_{i}\left(b_{i}+b_{i}^{\dagger}\right)\left(n_{i}-1\right)+\Omega_{0} \sum_{i} b_{i}^{\dagger} b_{i}
$$

The bosonic degrees of freedom can be integrated out in the path integral formalism, such that an effective interaction

$$
U_{\text {eff }}(\omega)=\frac{2 g^{2} \Omega_{0}}{\omega^{2}-\Omega_{0}^{2}}
$$

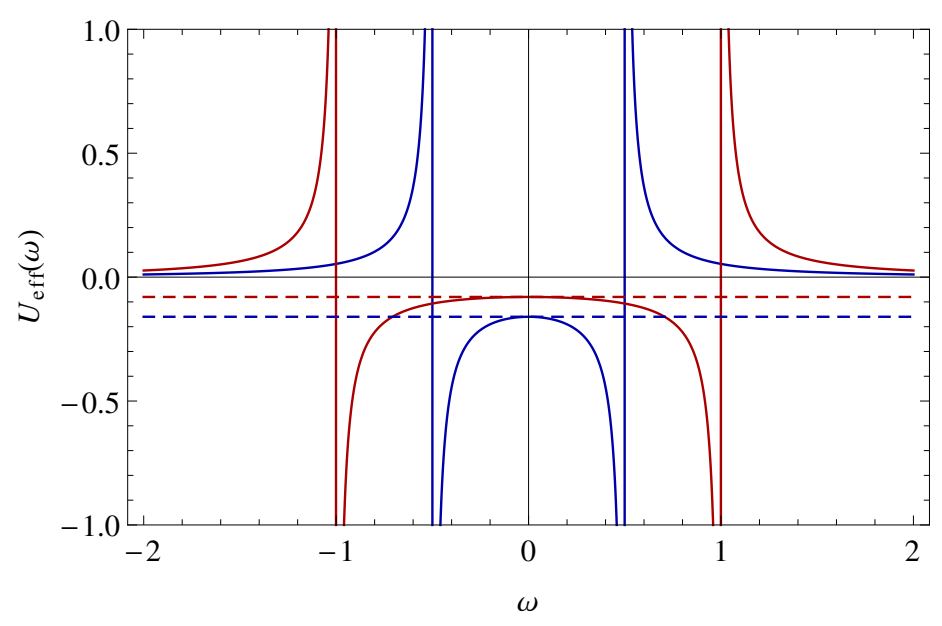

Figure 3.10: Effective interaction, Eq. (3.9), of the Holstein model with $g=0.2$ for $\Omega_{0}=$ 0.5 (blue) and $\Omega_{0}=1.0$ (red). The dashed lines show the effective attractive interaction in the corresponding anti-adiabatic limit. 
results [Fre93, Bau10a]. The bosonic bath may therefore be thought of as an effective retarded interaction among the conduction electrons.

This effective interaction is shown in (Fig. 3.10). For large $\omega$ it actually tends to zero while for $\omega \rightarrow 0$ it is indeed attractive, $U_{\text {eff }}=-\lambda$ with $\lambda=2 g^{2} / \Omega_{0}$. At $\omega= \pm \Omega_{0}$ the effective interaction diverges and changes sign from repulsive to attractive. In the anti-adiabatic limit $\Omega_{0} \rightarrow \infty$, keeping $\lambda$ constant, the Holstein model thus maps onto the attractive Hubbard model where the effective interaction between electron becomes instantaneous $U_{\text {eff }}(\tau) \rightarrow U \delta(\tau)$. In the opposite adiabatic limit $\Omega_{0} \rightarrow 0$ the phonons become static in the sense that its coordinate does not change in time, hence $U(\tau) \rightarrow U$ is constant. The Holstein model interpolates between these two limits [Fre93] in which the system exhibits either superconducting order in the anti-adiabatic limit, or a charge density wave (CDW) order in the adiabatic limit. In the absence of long range order and for large $\lambda \gg t$, the model shows a tendency towards bipolaron formation with a binding energy $U_{\text {eff }}=-\lambda$ [Bau10b].

The coupling of conduction electrons to Holstein phonons in the KLM has just recently gained attention [Nou09, Bod11]. In the PAM this type of coupling is extensively discussed in [Rac10]. In the anti-adiabatic limit the KLM with phonons maps to a KLM with a negative- $U$ correlation among the conduction electrons. Kondo screening and the coherence scale $T_{0}$ are efficiently suppressed in this case [Bod10] due to the increasing charge correlations. The observed suppression is actually stronger than exponential. The effect of phonons away from the anti-adiabatic limit is similar. As $T_{0}$ is a rather small energy scale, it is already substantially suppressed by a weak electron-phonon coupling [Rac10, Bod11. This interplay eventually results in a breakdown of the lattice Kondo effect when the energy scale of charge fluctuations is larger than the coherence scale, $U_{\text {eff }}>T_{0}$, or even due to the formation of polarons for large electron-phonon couplings.

In (Fig. 3.11) the DOS of the Holstein-Kondo-Lattice and the corresponding quasiparticle residue $Z$ for a fixed Kondo coupling $J=W / 5$ and fixed $\Omega_{0}=W / 2$ is presented as function of the electron-phonon coupling $g$. The phonons clearly lead to a reduction of the width of the pseudogap close to the Fermi energy and to a reduction of the overall effective bandwidth. At energies higher than the reduced bandwidth additional features appear with increased $g$, reflecting enhanced charge correlations around $\omega \approx U_{\text {eff. }}$. 


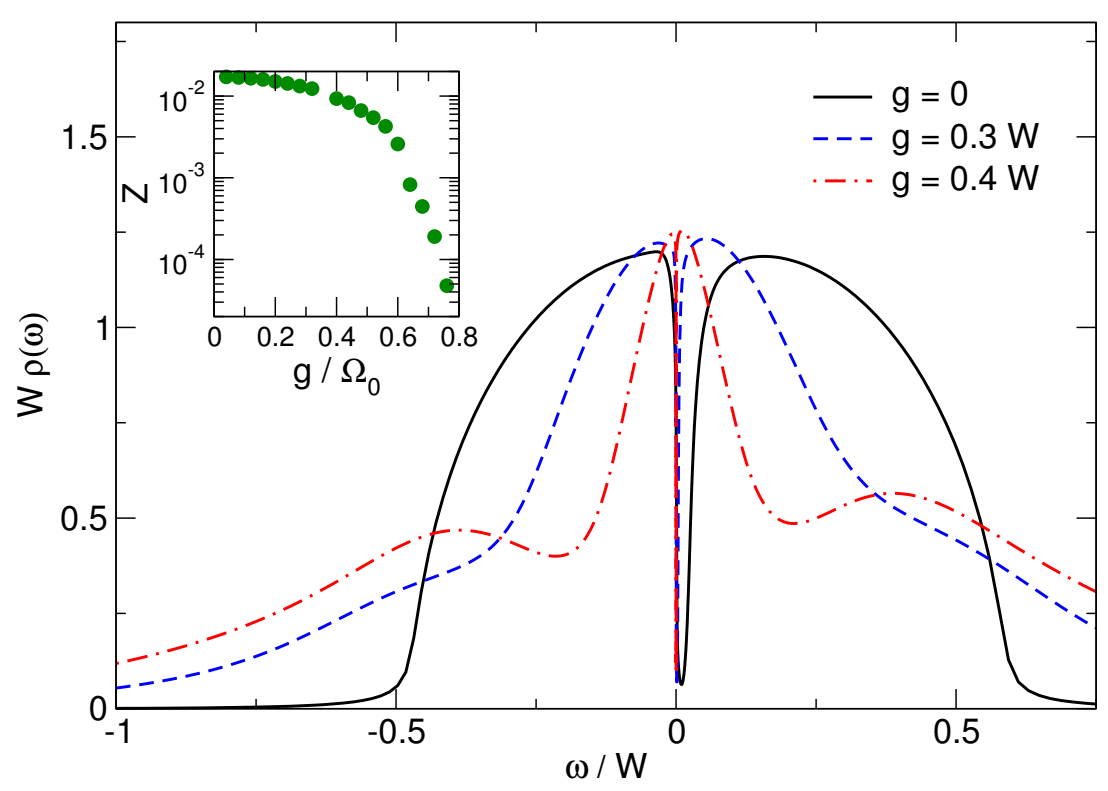

Figure 3.11: DOS of the KLM $(J / W=0.2)$ with an Einstein-phonon mode with frequency $\Omega_{0}=0.5 \mathrm{~W}$ for different electron-phonon couplings $g$. Inset: Corresponding renormalization factors for a larger range of electron-phonon couplings. 


\section{The Antiferromagnetic Phase of the Kondo-Lattice Model}

Antiferromagnetic order is frequently found in strongly correlated electron systems such as cuprates or HF materials at low temperatures [Sac12]. Quite often this AF phase appears close to a superconducting instability. For example, the undoped cuprate $\mathrm{La}_{2} \mathrm{CuO}_{4}$ is an antiferromagnetic insulator with a Néel temperature roughly at room temperature. Doping this compound with a small amount of hole carriers, e.g., by Ba, it becomes superconducting [Lee06]: $\mathrm{La}_{1.85} \mathrm{Ba}_{0.15} \mathrm{CuO}_{4}$ was the first high- $T_{c}$ superconductor discovered by Bednorz and Müller in 1986 [Bed86]. An example of an antiferromagnetic HF metal is the compound $\mathrm{CeCu}_{2} \mathrm{Si}_{2}$ which is extremely sensitive to small variations in stochiometry [Ste12b]. In samples with a tiny $\mathrm{Cu}$ deficit ('A-type') $\mathrm{AF}$ order is observed and with a tiny $\mathrm{Cu}$ excess ('S-type') the material becomes superconducting at low temperatures. For the nominal 1:2:2 stochiometry, a first-order transition from the AF to the superconducting state is observed by lowering the temperature [Ste79].

AF order in HF systems can be of either localized or itinerant type. For small exchange couplings, the weak antiferromagnetic order can qualitatively be explained by ordered localized moments, which are effectively decoupled from the conduction band but polarize the conduction electrons. This type of antiferromagnetism corresponds to a small Fermi surface since only the conduction-band states contribute to the Fermi volume. The strong coupling regime, on the other hand, is more intricate since the magnetic moments are more of an itinerant character and contribute to a large Fermi volume. The AF ordering then occurs in the heavy quasiparticle system. These itinerant "heavy-fermion antiferromagnets" typically tend towards incommensurate magnetic order. It is of high interest to classify antiferromagnetically ordering HF compounds and AF order emerging in corresponding models. The most interesting case is, of course, when a single compound can be tuned by a control parameter like doping, pressure or magnetic field to both types of AF order. This is intimately related to the type of the AF QCP as outlined in the last chapter.

On the theoretical side, the Doniach diagram already predicts the existence of an antiferromagnetic phase for materials which can effectively be described as Kondo lattices. It does, however, not give any hint to the actual nature of AF order within the model. Actually, the KLM shows a variety of magnetic phases [Lac79, Faz91] such as Néel antiferromagnetism, incommensurate magnetic phases and even a ferromagnetic (FM) phase is observed at 
strong doping. A peculiar feature in the FM phase was just recently observed [Pet12]: At strong doping the KLM orders ferromagnetically only in the majority channel while the minority electrons exhibit an insulating behavior. This sort of cooperation of ferromagnetism and Kondo singlet formation was termed spin-selective Kondo-insulator. Since the FM phase emerges in a region of the phase diagram complementary to a superconducting phase in the KLM [Bod13], FM ordering is not covered in this work.

Within DMFT the AF phase of the KLM has been studied in two and infinite dimensions [Ass99, Pet07]. Calculations in the Néel phase with a bipartite staggered magnetization can be easily carried out in single-site DMFT by introducing a bipartite structure [Zit02]. Incommensurate magnetic phases are, however, hard to stabilize in the simple DMFT approach [Pet09]. Nevertheless, there are at least indications to incommensurate ordering such as an increasing instability of the AF ordered phase or even an oscillating magnetization within DMFT iterations [Pet09, Bod11]. Both the nature of AF order itself and the possible competition with superconductivity is of large interest for an extended Doniach diagram.

In Sec. 4.1 DMFT+NRG results for the antiferromagnetic Néel phase of the half-filled KLM at zero temperature are presented. The presence of a recently observed resonance in the DOS is shown and related with the magnetization of conduction-band electrons. The next section 4.2 extends the discussion to the doped system close to half filling, where a metal insulator transition is observed as function of Kondo coupling. The insulating state is very fragile and found to be destroyed by small finite temperatures. The transition from a local-moment antiferromagnet with a small Fermi surface to an itinerant antiferromagnet with a large Fermi surface is discussed and related to the type of the QCP in the KLM.

The work in section 4.1 was initiated by Rok Žitko, who first observed the "spin resonance" in the conduction electron DOS in the AF state and provided the NRG code [Z̆]. Part of the work in section 4.2 has been published in Journal of Physics: Condensed Matter [Bod11] and therefore it may be, that some parts of the corresponding section resemble the manuscript Bod11]. The work was a collaboration with Rok Žitko, Robert Peters and Thomas Pruschke. 


\subsection{Half Filling: The Antiferromagnetic Kondo Insulator}

In the $\mathrm{AF}$ phase it is necessary to retain a bipartite $(A-B-)$ lattice structure and spindependence in the DMFT. Due to the symmetry $X_{A, \sigma}=X_{B, \bar{\sigma}}$ for spin-dependent quantities $X$ in the Néel AF phase it is sufficient to solve the effective two-site problem in a single spin-resolved NRG calculation. Throughout the chapter, the NRG calculations within each DMFT iteration are done with $z$-averaging [Ž $09 \mathrm{~b}], z=16$, a discretization parameter $\Lambda=2.1, N=1000, \ldots, 6000$ kept states, and with the full density-matrix approach Wei07. As the case of half filling is most stable within single-site DMFT, it is discussed first. At $n=1$ the KLM is an insulator which orders antiferromagnetically (AFI) below a critical coupling $J \lesssim J_{c}$, as expected from the Doniach diagram. Examples for the spin-resolved DOS in this AFI state are shown in (Fig. 4.1).

At half filling, the DOS for the majority spin (green) is fully symmetric to the one for the minority spin (red) and their sum yields the total spin-averaged DOS $\rho_{\text {tot }}=\rho_{\uparrow}+\rho_{\downarrow}$, which is also shown in (Fig. 4.1). The regime for small $J \lesssim 0.1$ can be fully explained by a Hartree approximation [Zit02]; in the majority-spin DOS (green) one observes a square-root singularity at the lower gap edge and a remnant of the power-law behavior at the upper gap edge. These characteristics begin to vanish upon increasing the Kondo coupling starting from $J / W=0.1$. For a larger $J / W=0.25$ the insulating gap has roughly doubled but the singularities are no longer present at the gap edges. One observes a new feature, instead, which is referred to as spin resonance or side resonance (SR) in the following; enlarging $J$, it splits from the gap edge and moves deep into the band. The shift of the SR initially scales with $J$. The majority-spin DOS features a spin resonance with a sharp low-energy edge within the $\omega<0$ part. The minority-spin DOS exhibits a corresponding dip feature and vice versa for positive frequencies. The $\mathrm{SR}$ can also be observed in the total spin-averaged DOS at this position, since the resonance is more pronounced than the dip.

The staggered magnetization of the conduction band is defined as $m_{c}=\left\langle S_{z}^{c}\right\rangle$ and similarly $m_{f}=\left\langle S_{z}^{f}\right\rangle$ is the polarization of the local moments. Due to the antiferromagnetic coupling $J>0$ both have a different sign. The sum $m_{\text {tot }}=m_{c}+m_{f}$ quantifies the net magnetization. For the weak-coupling case shown in the top panel of (Fig. 4.1) it is $m_{c}=-0.111$ and $m_{f}=0.469$, that is, the local moment system is strongly polarized while the staggered magnetization of the band states is rather weak. Indeed, one expects the largest $m_{f} \rightarrow 1 / 2$ and an unpolarized conduction band, $m_{c} \rightarrow 0$, for small $J \rightarrow 0$ since the system of the ordered local moments is then almost decoupled. For stronger coupling around $J / W=0.25$, instead, $m_{f}$ is reduced by more than $50 \%$ while $m_{c}$ is only slightly less than in the case of $J / W=0.1$. In general, the absolute value of $m_{c}$ is mostly only a fraction of $m_{f}$, but seems to become comparable close to $J_{c}$. This leads to a strongly reduced total moment for $J \lesssim J_{c}$ which can be understood due to the increasing tendency towards Kondo-singlet formation. In fact, the critical $J_{c} \gtrsim W / 4(=t)$ lies roughly in the region where $J$ exceeds the hopping parameter $t$. The magnetizations as a function of $J$ are shown in (Fig. 4.2). 


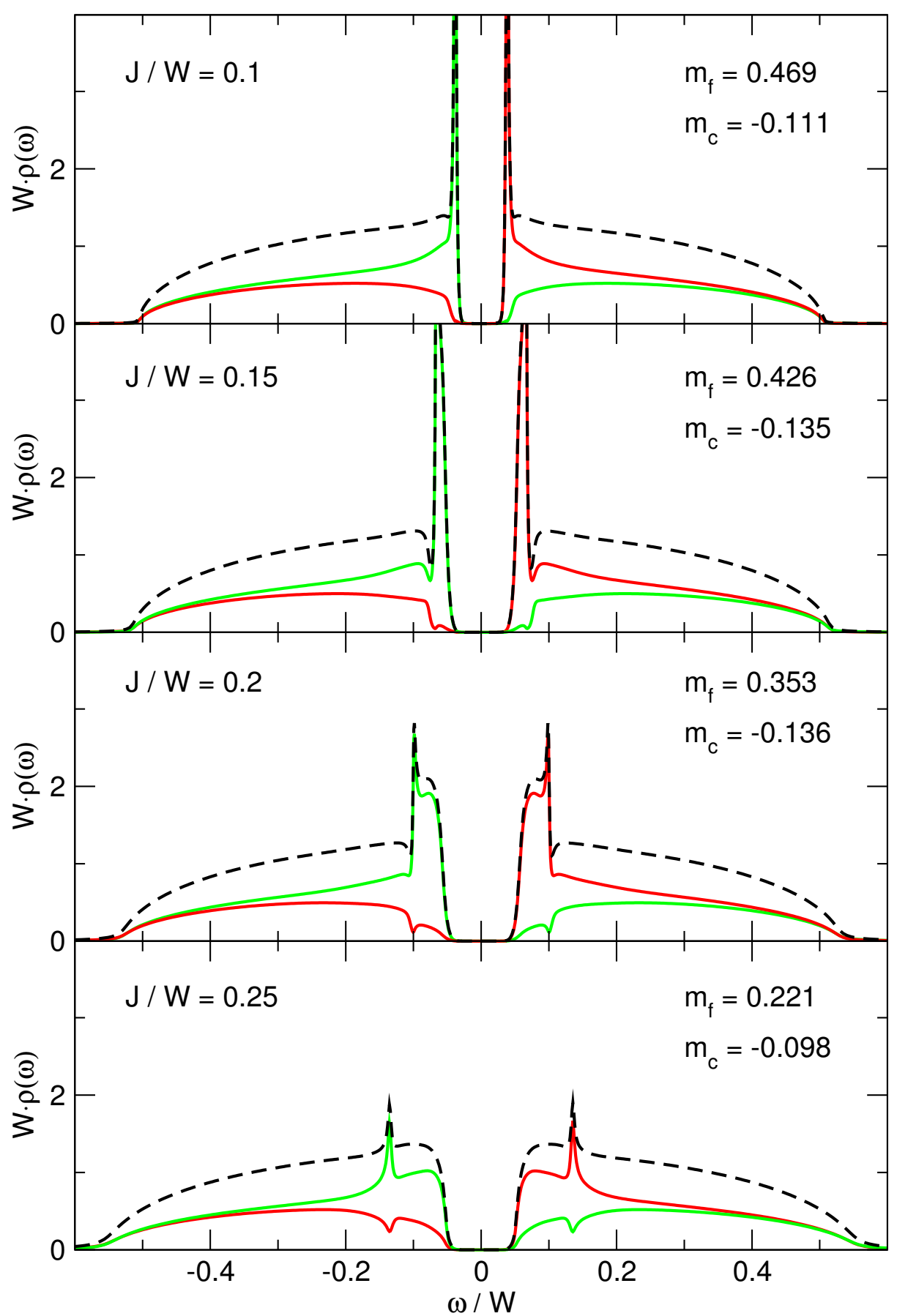

Figure 4.1: Spin-up (green), spin-down (red) and total (black) DOS of the KLM in the AF ordered phase for different couplings $J / W=0.1,0.15,0.2,0.25$ at half filling. $m_{c}$ and $m_{f}$ are the corresponding staggered magnetizations of the conduction (c) band electrons and local moments (f). 


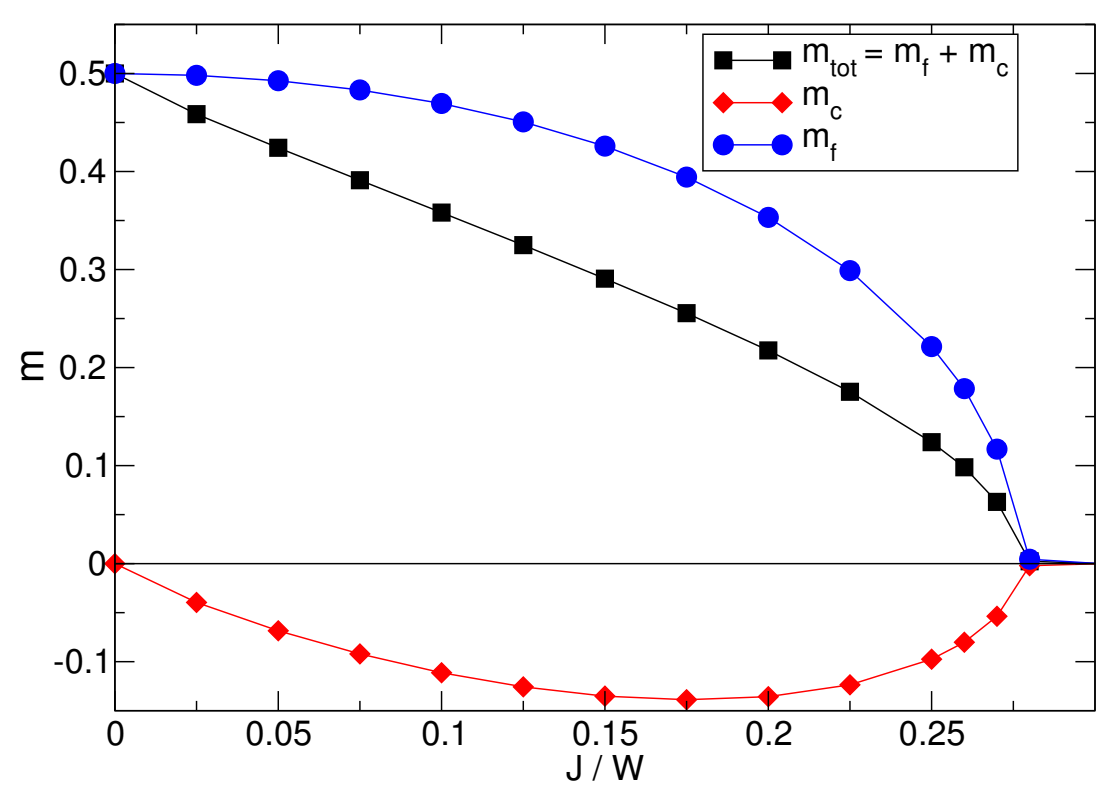

Figure 4.2: Magnetizations for the Néel-ordered KLM at half filling.

From (Fig. 4.2 the critical coupling is determined as $J_{c} \approx 0.275 W$. Due to the opposite signs of $m_{c}$ and $m_{f}$, the total staggered magnetization is monotonously decreasing as $J$ is increased towards $J_{c}$. In the region of $J$ where $m_{c}$ is maximal, i.e. around $J / W \approx 0.175$, the spin resonance in the DOS is the most pronounced, cf. (Fig. 4.1).

\subsection{Antiferromagnetism away from Half Filling}

Away from half-filling the DMFT suffers from convergence problems in the AF phase of the KLM. In order to obtain a reasonable convergence, the DMFT needs to be supplemented with Broyden mixing [Ž09a. Even then, in certain parameter regimes, which are likely to be connected to a spin-density-wave groundstate, it is not possible to stabilize the DMFT calculations. While the small- $J$ region can be stabilized for a filling of $n=0.9$, it becomes increasingly hard to get converged results for $J / W \gtrsim 0.1$. It is nevertheless possible to obtain converged results for certain other fillings with the same $J$.

In order to cover the range of relevant couplings, the DOS is plotted for two different fillings $n=0.9$ and $n=0.94$ in (Fig. 4.3). The essential features in the spectral functions 
are expected to be only quantitatively different for the two close-by filling factors. For $J / W=0.075$ the remnant AF (pseudo-) gap is confined by asymmetric square-root singularities, the one stemming from the majority-spin part (green) being damped. The gap position has shifted to finite positive frequencies in contrast to half filling. At $\omega=0$ instead, a tiny resonance feature for spin-up and a dip for spin-down develops. This very feature becomes actually more pronounced upon increasing the Kondo coupling to $J / W=0.125$ : The DOS of the majority electrons shows a high and narrow resonance very close to the Fermi energy, $\omega \lesssim 0$, cf. inset of (Fig. 4.3). This singularity seems to be accompanied by an adjacent shoulder which drops down to zero weight for $\omega \rightarrow 0^{-}$. For non-negative frequencies a gap is seen in the spin-up DOS. For the minority electrons, the DOS exhibits only little spectral weight at the peak position and does also feature a gap for $\omega \geq 0$. The total DOS thus classifies the system as an insulator for $n=0.9$ and $J / W=0.125$ since the Fermi energy is gapped. At a small finite temperature $T / W=0.001$, the insulating state is, however, lifted to a metallic state ${ }^{1}$, as studied in Ref. [Hos13. Opposed to the emerging resonance close to $\omega=0$, the AF gap diminishes to a small dip-hump feature from $J / W=0.075$ to $J / W=0.125$. The structures become more apparent in the panel for $J / W=0.175$, though $n=0.94$ in this case. In the temperature-induced metallic case the strong resonance close to the Fermi energy might be interpreted as a spin-selective coherence effect. Indeed the effective masses and thereby coherence scales $T_{0}$ of both spin channels can differ by several orders of magnitude in the metallic case of finite temperatures for this parameter regime [Hos13]. Finally, for $J / W=0.225$ the features are developed on an even larger scale: The shoulder adjacent to the SR is clearly visible in the majority DOS. In the minority-electron DOS one can now observe a semi-circle-like spectral weight below the resonance. The gap above the Fermi energy grows with increasing $J$ and the remnant AF singularities are almost washed out.

The magnetization of local moments given in (Fig. 4.3) drops by more than $50 \%$ from $m_{f}=0.457$ to $m_{f}=0.167$ by increasing $J / W=0.075$ to 0.125 . Neither this fast decrease nor a phase transition from metallic to insulating is expected from the half-filled case. In (Fig. 4.4) the different magnetization observables for a fixed filling $n=0.9$ are shown as function of $J$. Indeed, the magnetizations seem to confirm a transition between two different AF regimes: $m_{f}$ starts with the maximal polarization at weak coupling and is only slightly reduced up to $J / W=0.075$. In a small parameter window up to $J / W=0.1$, it is reduced by more than $50 \%$ - while $m_{c}$ saturates for $J \lesssim 0.1$. Due to the small scale of $m_{c}$, the total magnetization essentially follows the large $m_{f}$. In the region $0.1<J \leq 0.125$, converged results are obtained for occupations differing by $\pm 0.2 \%$ from $n=0.9$. This may lead to the somewhat wiggling magnetizations shown in the figure. Nevertheless, the results definitely hint at a phase transition at $J / W=0.1$ within the AF phase. The DMFT does not converge in the parameter region $0.125 \lesssim J / W \lesssim 0.25$, but for $J / W \geq 0.25 \mathrm{AF}$ order definitely vanishes.

1 The corresponding data are not shown here. 


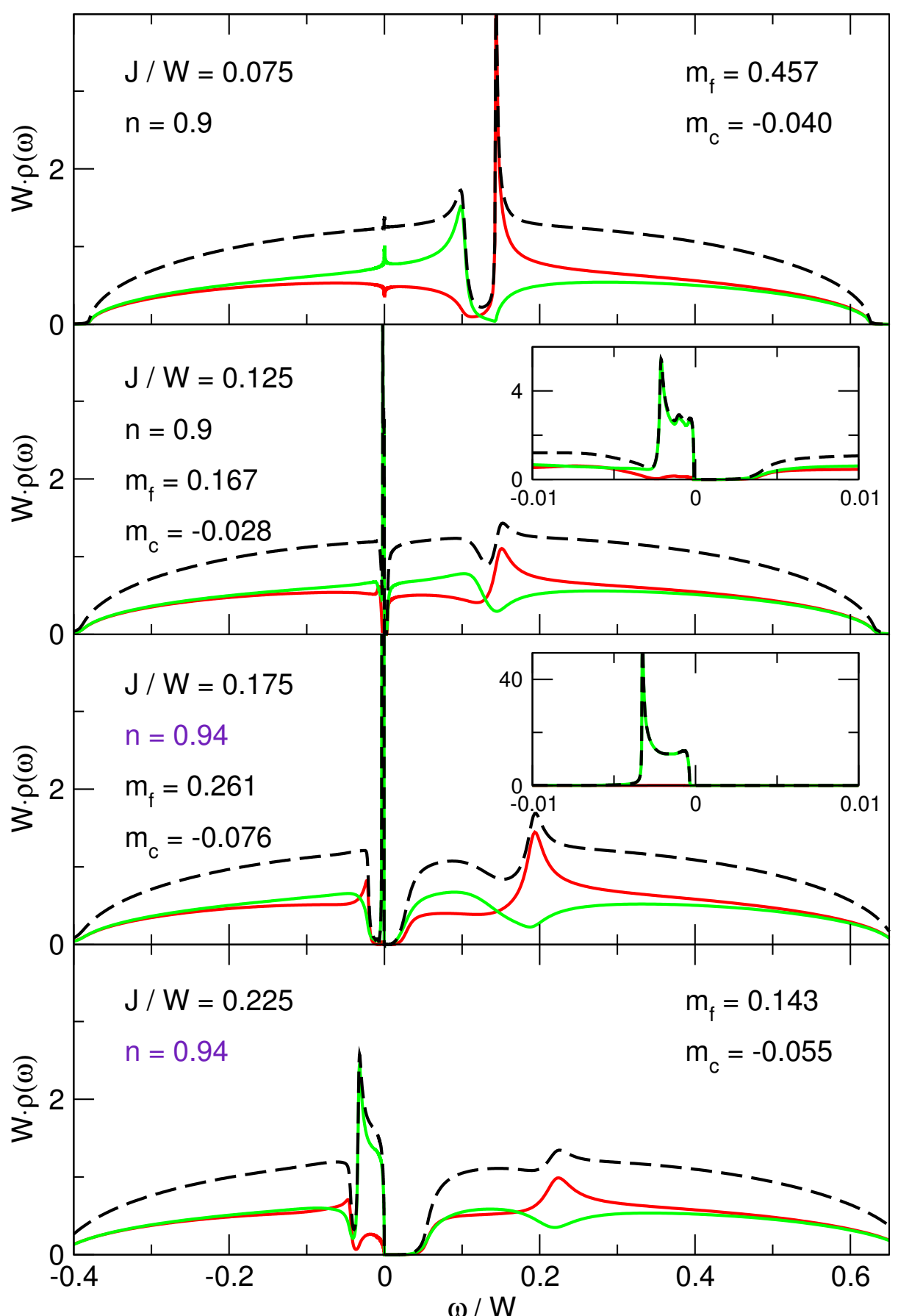

Figure 4.3: Spin-up (green), spin-down (red) and total (black) DOS of the KLM in the AF ordered phase for different couplings $J / W=0 ., 0.15,0.2,0.25$ away from half filling. Note that the upper two and lower two panels are at different fillings $n=0.9$ and $n=0.94$, respectively. The emerging peak close to the Fermi energy is magnified in the insets. 


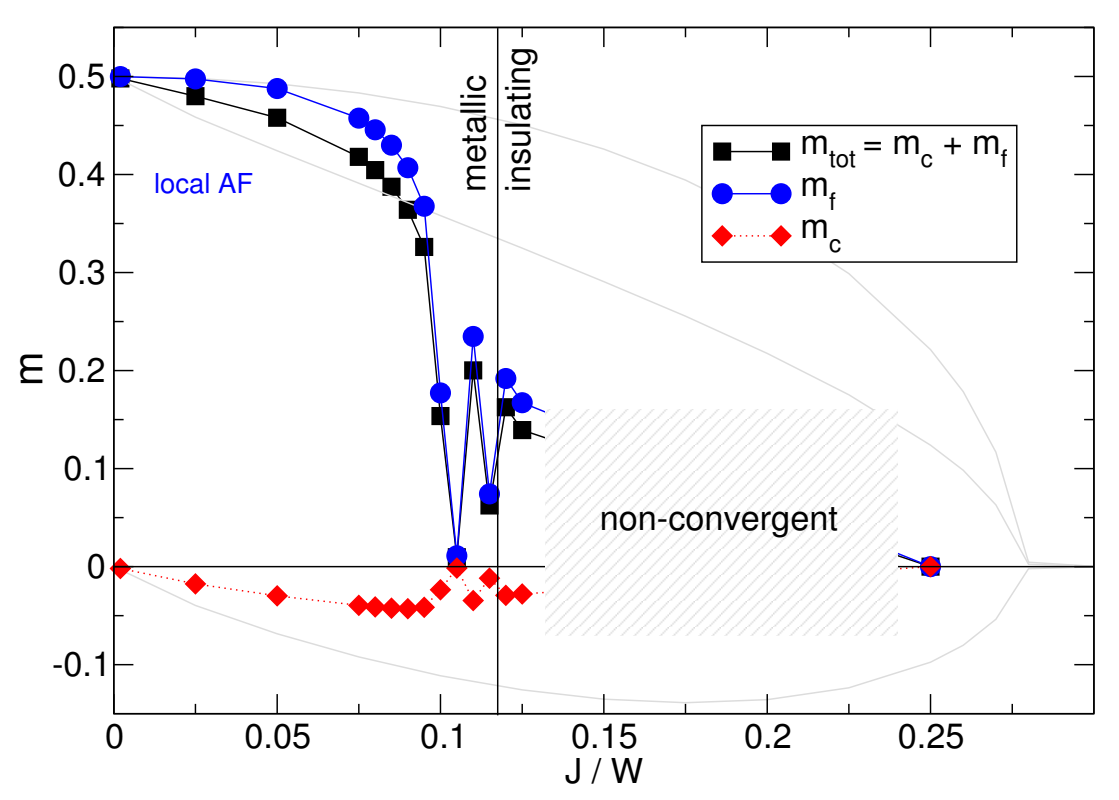

Figure 4.4: Magnetizations in the KLM for $n=0.9$. The local-moment antiferromagnetism breaks down for $J / W \approx 0.1$ and a metal-insulator transition is observed at $J / W \approx 0.2$. The region of non-convergent results is shaded in light gray and the results for half filling are also shown for comparison in light gray.

For even higher Kondo couplings, $0.125<J<J_{c}$, it was not possible to stabilize the DMFT in the $\mathrm{AF}$ phase for the fixed filling $n=0.9$ up to $J_{c} / W \lesssim 0.25$. For $J=0.25$ a vanishing magnetization is found, such that $J_{c} \approx 0.25 \mathrm{~W}$. Despite the lacking convergence in the region of interest, $0.125<J / W<J_{c}$, the DMFT calculations hint at an incommensurate AF order therein. This instability is likely to be lifted by an additional Heisenberg exchange coupling $J_{H}$ between the local spins [Hos13]. In Ref.[Hos13, a transition from the localmoment AFM to itinerant heavy-fermion AFM is observed at $J_{c, 2}=0.16 \mathrm{~W}$ for a filling $n_{c}=0.95$ and a small $J_{H} / W=0.025$. These parameters are consistent with a larger $J_{c, 2}$ compared to the present case. A kink in the staggered magnetization at $J_{c, 2}$ was also confirmed in Ref. [Hos13, which develops to a rather steep drop in $m_{\text {tot }}$ for $T \rightarrow 0$.

Measurements of the Hall coefficient in the $\mathrm{HF}$ material $\mathrm{YbRh}_{2} \mathrm{Si}_{2}$ show a steep change upon varying an external magnetic field. Lowering the temperature, this crossover becomes much sharper and converges to the field-induced AF QCP [Pas04, Geg08]. The extrapolated zero-temperature jump is inconsistent with a SDW-type QCP where the Hall coefficient is continuous across the QCP [Nor03]. The results rather prove a local QCP in this material, 
i.e., the Fermi surface collapses together with the onset of AF order. In variational Monte Carlo calculations for the Kondo lattice such a discontinuous change is found for a filling $n_{c} \lesssim 0.82$, but for larger fillings there appear two separate types of transition Wat07]: Decreasing $J$ from a large value, the ground state changes from paramagnetic to antiferromagnetic with an intact Kondo effect, that is, a large Fermi surface. This transition is identified as second-order and the Hall-coefficient changes continuously. Lowering $J$ further, the AF ordered groundstate changes its Fermi surface topology from "large" (hole-like) to small (particle-like) in a first-order transition [Wat07], accompanied by a discontinuous change in the Hall coefficient.

Within the DMFT treatment of the KLM the AF QPT at half filling was identified to lie inside the HF regime [Hos10] and does not feature a localization of the $f$ spins. Away from half filling, the situation changes: Including an explicit small RKKY interaction $J_{H}$ in the model, an itinerant-localized transition of the local moments is found well inside the AF ordered regime Hos13. The latter is thus divided into weak itinerant antiferromagnetism in the conduction band for small $J$, and a heavy-fermion antiferromagnet for larger $J$. It is thus of interest if this behavior persists for $J_{H}=0$ in the pure KLM. From the $T=0$ results shown in (Fig. 4.3) and (Fig. 4.4) one can expect such a transition at $J_{c, 2} / W=0.1$ for $n=0.9$, but these results are not totally conclusive. However, from the results in Hos13] the transition can be expected roughly at this coupling strength since the somewhat higher value of $J_{c, 2} / W=0.16$ is likely due to a slightly higher filling, finite temperature and an additional intersite coupling $J_{H}$.

More evidence for the type of AF phase is given by inspection of the momentum-resolved spectral function $A_{\sigma}\left(\varepsilon_{\mathbf{k}}, \omega\right)$. In the Bethe lattice the definition of a momentum is lacking, but one can resolve the general energy $\varepsilon$-dependence. The corresponding spectral functions to the majority-electron DOSs (upper panels in (Fig. 4.3) ) are shown in (Fig. 4.5). The overall structure for $J / W=0.075$ looks rather linear with broader and more pronounced peaks for $\omega<0$. For $\omega \approx 0.1$ the remnant AF pseudogap is still visible as a lack of spectral weight. In the close-up the emerging resonance at $\omega=0$ is clearly visible as a bended part of the upper half $(\omega>0)$ of the band. It is actually a precursor of the hybridized bandstructure, but intersects the Fermi energy such that a small Fermi surface results. The situation changes completely for $J / W=0.125$ where the hybridized bandstructure and thereby the heavy Fermi liquid fully developed. Although $A_{\sigma}(\varepsilon, \omega)$ now exhibits an insulating gap, it is lifted by a small finite temperature (cf. discussion above) and results in an itinerant metallic antiferromagnet, cf. (Fig. 4.4). These results clearly hint towards the spin-density-wave nature of the QCP in the Kondo-lattice model. Similar results are published in [Bod11]. 

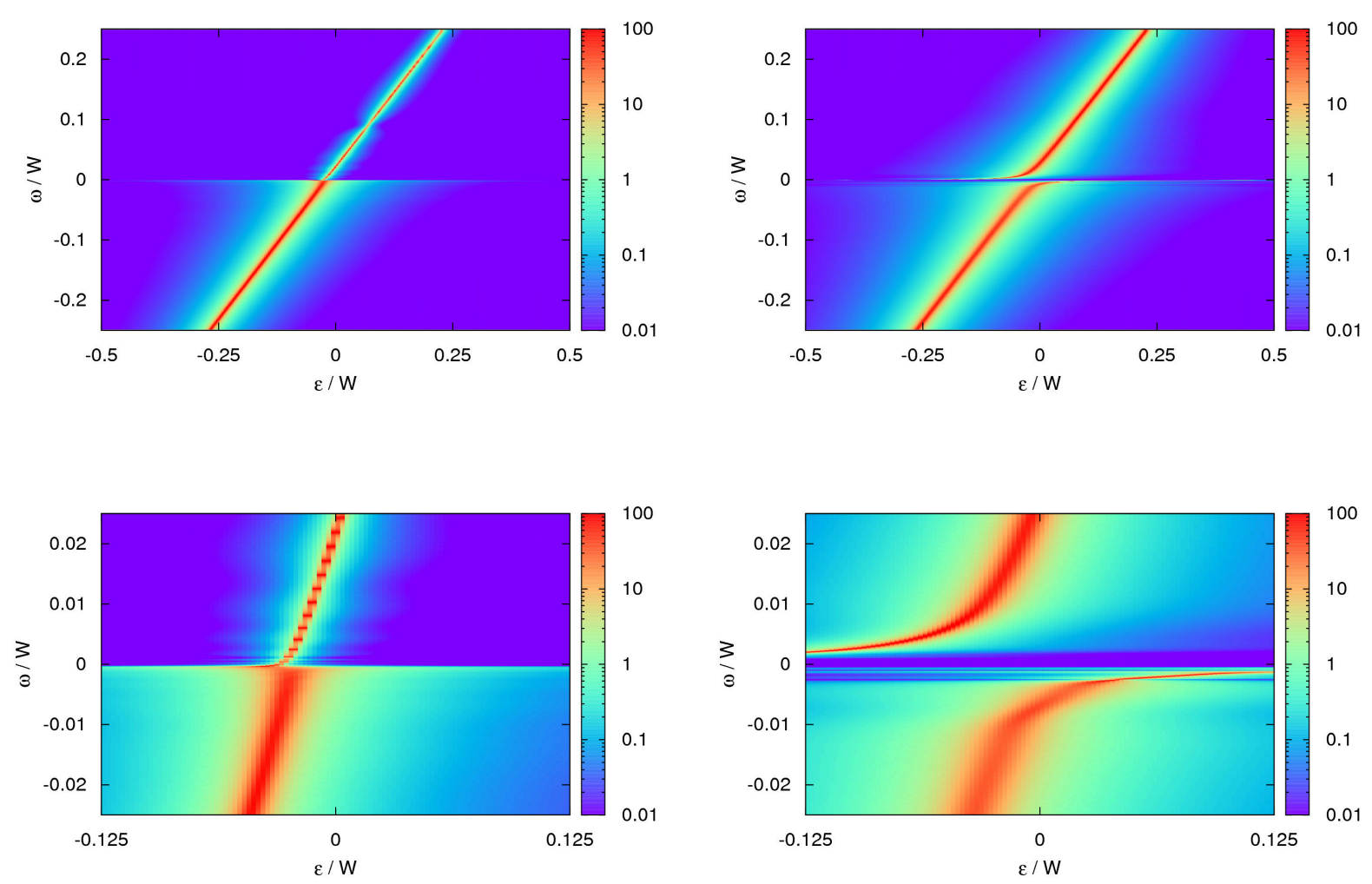

Figure 4.5: Spectral functions $A_{\sigma}(\varepsilon, \omega)$ for spin-up electrons (KLM, $\left.n=0.9\right)$. Left column: $J / W=0.075$. Right column: $J / W=0.125$. Note the logarithmic color scale.

\subsection{Summary}

Generic examples of the Kondo-lattice model in the antiferromagnetic phase at $T=0$ were investigated for the Bethe lattice in infinite dimensions by means of DMFT in combination with NRG. While the magnetization behavior of the KLM is known, the spectral detail of the newly observed "spin resonance" was not discussed in the literature before. At half filling its strength correlates with the polarization of the conduction band. Close to half filling, AF order persists in a metallic or even insulating state at zero temperature: A transition from the local-moment to itinerant antiferromagnetism is not observed at $T=0$ but rather a transition from an AF metal to an AF insulator is found. This transition happens when the bands are fully hybridized. The DOS exhibits then a resonance in the majority channel very close to the Fermi energy at negative frequencies. Thus, the insulating state is lifted by a small finite temperature which broadens the steep slope and yields a finite spectral weight at $\omega=0$. The values of the DOS at the Fermi energy for each spin channel can nevertheless differ by a factor of up to 30 for small finite temperatures. 
The AF order in the KLM typically appears close to half filling where the AF QCP is, within DMFT, identified to be of SDW-type. Since SDW-type QCPs are mostly hidden by a superconducting dome, it seems promising to search for superconductivity in the phase diagram of the KLM. A possibly important finding with regard to superconductivity is the strong enhancement of the local magnetic susceptibility $\chi_{\text {loc }}$ at low temperatures near the itinerant-localized transition, but well in the itinerant regime [Hos13]. Due to the immediate connection between local and RKKY fluctuations, cf. Eq. (3.3), these fluctuations may provide a "pairing glue" not only for isotropic, but also for anisotropic superconductivity. Even in a single-site DMFT, where only $s$-wave superconductivity can be captured, the search for superconductivity close to the localized-itinerant transition surprisingly turned out to be successful [Bod13] and is discussed in the next chapter. 



\section{Unconventional Superconductivity in the Kondo Lattice}

In addition to extreme Fermi liquid properties, various phase transitions and symmetrybreaking phases are observed in HF materials. They either occur within the heavy Fermi liquid, as revealed by thermodynamics, or compete with it [Ste84, Loe07]. An example of the former class is the SDW-type antiferromagnetic phase transition discussed in the previous chapter. Detailed investigation of the ordered states uncovered them as being of unconventional nature Gre91. This observation has been underpinned by the development in the field over the past two decades which manifested in the discovery of a large number of HF systems exhibiting curious quantum phase transitions, some of them recognized as being closely connected to superconducting order [Ste01, Loe07].

HF superconductivity is usually found to emerge out of the HF liquid Geg08 and not close to local QCPs, which are accompanied by a breakdown of the Kondo effect. This observation seems on the one hand plausible, since local magnetic moments and superconductivity can only coexist under very restrictive conditions on the microscopic scale. Superconducting order in HF systems should thus emerge when the local moments are "dissolved" in the system of heavy quasiparticles and do no longer act as localized magnetic moments. On the other hand, quantum critical fluctuations of the order parameter are also identified to be responsible as the "glue" for Cooper pairing. They also appear at local QCPs, in addition to quantum critical fluctuations of the Kondo breakdown and the Fermi surface transition. From this point, it is not immediately obvious, why the type of QCPs provides such a clear-cut criterion Geg08. The appearance of superconductivity in these compounds is a rather unconventional feature since magnetic order seems to be inevitable for it to appear. A conclusive experimental confirmation of this fact has just been accomplished recently: By inelastic neutron scattering measurements in the prototypical HF superconductor $\mathrm{CeCu}_{2} \mathrm{Si}_{2}$ antiferromagnetic excitations are identified as the driving force behind superconductivity close to the AF QCP [Sto11].

To theory, HF superconductivity is a rather long-standing challenge. To include superconductivity, models for HF systems such as the KLM or PAM usually need to be complemented by additional bosonic degrees of freedom [Raz84, Gre84] or new concepts like anisotropic composite pairing in multi-channel Kondo-lattice models [Fli08, Fli10]. In contrast, studies of the pure single-band Kondo-lattice or periodic Anderson model had 
not yielded conclusive evidence for superconducting ground states therein. Within DMFT, a hint to a local even-frequency superconducting instability in the PAM was found in [TZ98], though it is found to arise out of the normal state and not from a Fermi liquid. Static mean-field descriptions of the KLM or PAM are also found to yield superconducting solutions, either conventional pairing in the simple KLM [Gus00, or rather unconventional superconductivity in the PAM [Mas13] or extended Anderson-Kondo lattice [How12]. The validity of static mean-field treatments is, however, difficult to asses since fluctuations beyond mean-field pairing may destroy electron pairing. The DMFT and its cluster extensions Geo96, Mai05, map the lattice problem onto an impurity model, thereby loosing non-local correlation effects beyond the spatial size of the impurity cluster. To properly describe HF superconductivity within DMFT-based approaches, it is thus commonly believed that either large enough clusters are required or even a bath in the two-particle channel has to be included - which explicitly models a bosonic "glue" for superconductivity.

As a surprising finding during the course of this thesis, a robust $s$-wave superconducting solution for the KLM within the DMFT+NRG approach has been found, without any external glue [Bod13]. This SC phase is stable over a large region of the $(n, J)$-phase diagram. Although being of $s$-wave type, the pairing is driven by local spin fluctuations and the gap function is strongly frequency dependent. This novel type of pairing is thus highly unconventional. Both the formation of heavy quasiparticle bands and strong local spin fluctuations are identified to be a prerequisite for the superconducting order to emerge.

Section 5.1 starts with a general discussion of theoretical treatments of HF superconductivity before the bandstructure in the SC phase of the KLM is studied by means of both static and dynamical mean-field theory. In the following Sec. 5.2 dynamic and static properties in the SC phase at $T=0$ are studied in more detail. The focus is especially on the relation between pair correlations and the heavy Fermi liquid. Results for finite temperatures are shown in Sec. 5.3. Finally, in Sec. 5.4 the pair-mechanism for the present type of superconductivity is identified as originating from local spin fluctuations.

Much of the content in sections 5.2 - 5.4 is published in Physical Review Letters Bod13 and some parts of this chapter may thus resemble the manuscript. The work was done in collaboration with Rok Žitko, Matthias Vojta, Mark Jarrell and Thomas Pruschke. 


\subsection{Superconductivity in the KLM}

Early after the discovery of HF superconductivity theoretical attempts were made to understand how a Cooper pair condensate can emerge out of the heavy Fermi liquid. The theoretical studies included possible phononic origins of pairing in the Kondo lattice, potentially connected to the Kondo volume collapse [Raz84, Gre84], or pairing of $f$ electrons in the Anderson model with a small attractive interaction among $f$ electrons of unknown origin Ohk84. It turned out, that even $s$-wave superconductivity in the $f$-system would be anisotropic ${ }^{1}$ : It can thus also lead to rather unconventional features such as a gap function featuring line nodes on the Fermi surface Ohk84.

Since HF superconductivity was early associated with an unconventional pairing mechanism, it has in fact most often been assumed to appear among the $f$-electrons. Thus, the most promising model on this route is the periodic Anderson model, sometimes modified such that the $f$-electrons obtain a small dispersion $\varepsilon_{\mathbf{k}}^{f}$ around the average $f$-level $\varepsilon_{f}$, i.e., the kinetic energy term for the $f$-electrons reads

$$
H_{\mathrm{kin}}^{f}=\sum_{\mathbf{k} \sigma}\left(\varepsilon_{\mathbf{k}}^{f}+\varepsilon_{f}\right) f_{\mathbf{k} \sigma}^{\dagger} f_{\mathbf{k} \sigma}
$$

The possibility of superconductivity in this Yoshimori-Kasai model [Yos83] has been studied early after the discovery of the first $\mathrm{HF}$ superconductors $\mathrm{CeCu}_{2} \mathrm{Si}_{2}$ and $\mathrm{UBe}_{13}$ and gave first theoretical insight into the differences of HF superconductivity to conventional BCS theory [Tac84, Xu 87]. Some important issues of these studies should be noted here. It is assumed that $U,\left|E_{f}\right|, V$ are rather large such that the system constitutes a Kondo lattice but charge fluctuations are still allowed. Moreover, it is assumed that Cooper pairing takes place among $f$-electrons since the corresponding critical temperature seemed to be much higher than for $c$-electrons. ${ }^{2}$ While in BCS theory a frequency cutoff at the Debye frequency $\omega_{D}$ is introduced, in HF systems the attractive interaction between the heavy quasiparticles is limited to a frequency range up to the Kondo temperature, $\omega \sim T_{K}$. Above $T_{K}$, the Kondo effect leads to a repulsion of the electrons. The replacement of $\omega_{D}$ by $T_{K}$ yields a critical temperature proportional to the Kondo temperature [Tac84]

$$
T_{c}=1.13 T_{K} \exp \left(-\frac{1}{g_{c / f} \rho_{0}(0)}\right),
$$

where $g_{c / f}$ is the renormalized BCS coupling constant for either conduction band electrons or $f$ electrons. The DOS of the conduction electrons at the Fermi energy is denoted by $\rho_{0}(0)$. One would thus already expect a rather low $T_{c}$ since typically $T_{K} \ll \omega_{D}$. Another

1 In two dimensions, an extended $s$-wave symmetry of the gap function is given by $\Delta_{\mathbf{k}}^{s}=$ $\Delta_{0}^{s}\left(\cos \left(k_{x} a\right)+\cos \left(k_{y} a\right)\right)$, where $a$ denotes the lattice constant.

2 Recent theoretical studies of HF superconductivity in fact focused on $c$ - $f$-pairing, see e.g. [Mas13]. 
important difference to BCS theory is that the gap function is not equal to the order parameter $\mathrm{Xu} 87$ ].

\subsubsection{Static Mean-Field Description of HF Superconductivity}

Before the discussion of superconducting order emerging in the dynamical mean-field approximation for the KLM, it is instructive first consider the static MF treatment of superconductivity in the KLM. The MF description of the KLM in the paramagnetic phase has been introduced in chapter 3, cf. Eq. (3.5). The $f$ degrees of freedom introduced there mainly contribute to the flat and weakly dispersive parts of the band. In case of a superconductor, these very parts of the band are of particular interest as will be shown in the following. As a first simple model, a mean-field BCS-type pairing term can be added to the Hamiltonian in Eq. Eq. (3.5) both in the conduction $(c-)$ and $f$-band. Another possibility is to add a hybrid $c$-f-pairing term $\Delta_{c f}$. The latter type of pairing was recently proposed to explain $s$-wave superconductivity in HF systems [Mas13]. The general mean-field Hamiltonian for superconductivity in the Kondo-lattice can be written in a composite $(c-f) \otimes$ (particle-hole) structure:

$$
H_{\mathrm{KLM}}^{\mathrm{SC}-\mathrm{MF}}=\sum_{\mathbf{k} \sigma}\left(\begin{array}{llll}
c_{\mathbf{k} \sigma}^{\dagger} & c_{-\mathbf{k} \bar{\sigma}} & f_{\mathbf{k} \sigma}^{\dagger} & f_{-\mathbf{k} \bar{\sigma}}
\end{array}\right)\left(\begin{array}{cccc}
\varepsilon_{\mathbf{k} \sigma} & \Delta_{c} & V & \Delta_{c f} \\
\Delta_{c} & -\varepsilon_{-\mathbf{k} \bar{\sigma}} & \Delta_{c f} & -V \\
V & \Delta_{c f} & \varepsilon_{f} & \Delta_{f} \\
\Delta_{c f} & -V & \Delta_{f} & -\varepsilon_{f}
\end{array}\right)\left(\begin{array}{c}
c_{\mathbf{k} \sigma} \\
c_{-\mathbf{k} \bar{\sigma}}^{\dagger} \\
f_{\mathbf{k} \sigma} \\
f_{-\mathbf{k} \bar{\sigma}}^{\dagger}
\end{array}\right)
$$

Although it should be noted that all three types of off-diagonal order lead to qualitatively similar quasiparticle bands and gap structures, here the focus is, however, on a $c$ - $f$ pairing term. The corresponding bandstructure for a non-zero $\Delta_{c f}$ is shown in (Fig. 5.1), illustrating several new features in comparison to the paramagnetic case in (Fig. 3.4). First of all, due to the introduction of hole degrees of freedom, the Hamiltonian matrix has four eigenvectors and thus the number of quasiparticle bands is doubled. On the one hand, a pair of narrow particle- and hole-like bands is present around the Fermi energy. They originate from the formerly non-dispersive $f$-level $\varepsilon_{f}$. On the other hand, a corresponding pair of wide dispersive bands stemming from the $c$-electrons is present. The introduction of an off-diagonal superconducting order parameter (in this case $\Delta_{c f}$ ) has two effects. First, it introduces a full superconducting gap $\Delta_{0}$ around the Fermi energy. Secondly, it also gaps the narrow flat bands from the upper and lower dispersive band, respectively, at $E_{\mathbf{k}} \approx 0.1$ in (Fig. 5.1). The corresponding "side-gap" will be referred to as $\Delta_{>}$. Heavy-fermion pairs should appear as a pronounced weight in the flat particle and hole band close to the Fermi energy, with a full gap $\Delta_{0}$. The connection of the gap $\Delta_{>}$to Cooper pairing is, however, far from clear.

Actually, the gap ratio $\Delta_{0} / \Delta_{>}$can be tuned by using different mean-field parameters 


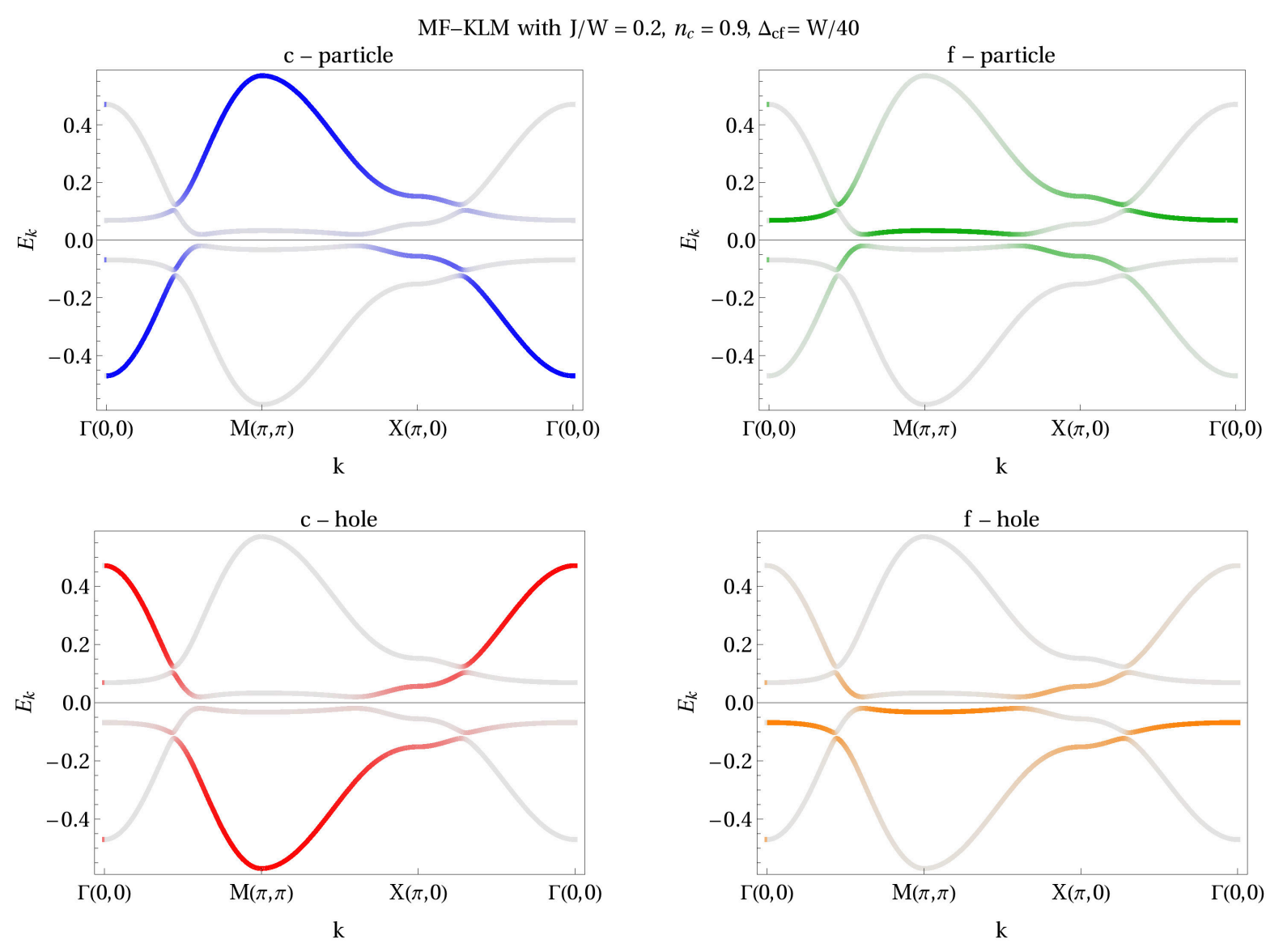

Figure 5.1: Typical renormalized quasiparticle bandstructure of the paramagnetic KLM on the two-dimensional square lattice in the mean-field picture. Left: Weight of $c$-states. Right: Weight of $f$-states. The color intensity represents the amount of weight and gray corresponds to zero weight.

$\Delta_{c}, \Delta_{f}$ and $\Delta_{c f}$, even to an almost vanishing $\Delta_{>}$by increasing $\Delta_{f}$ to high values together with a finite $\Delta_{c f}$. Due to quasiparticle scattering and finite lifetime effects, the gap $\Delta_{>}$is also likely to be filled. Below, these structures will be investigated in more detail by means of DMFT.

A similar effect in the bandstructure emerges in the AFM phase of the KLM (cf. Ch. 4), where the mean-field Hamiltonian can be written formally equivalent to (5.1). There the gapped structure of four bands in combination with finite lifetime effects eventually lead to the observation of the spin resonance in the DOS, cf. (Fig. 4.1). However, due to the non-zero imaginary part of the self-energy, $\operatorname{Im} \Sigma$, the avoided crossing appears as a simple crossing and the spectral density in-between is either enhanced or reduced, depending on sign of $\omega$ and spin, which eventually produces the resonances. A similar observation can thus be expected for DMFT results in the SC phase. 


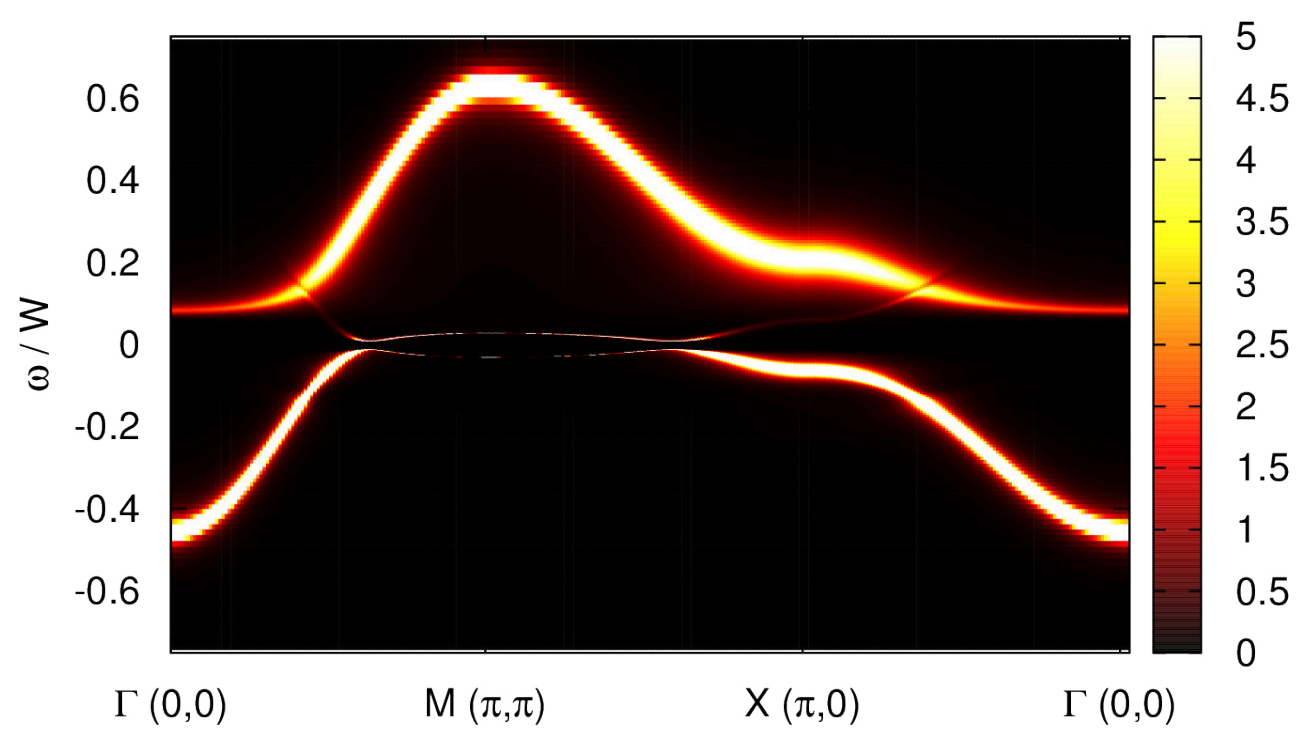

Figure 5.2: Spectral function $A\left(\varepsilon_{\mathbf{k}}, \omega\right)$ for the KLM in the SC phase at a filling $n=0.9$ and with $J / W=0.2$. A two-dimensional square-lattice DOS and dispersion is used for the plot.

For a comparison of the dispersion obtained in a static MF treatment, a brief "preview" to DMFT results shall be discussed at this point already. In figure (Fig. 5.2) a typical spectral function $A_{\mathbf{k}}(\omega)$ for the superconducting phase of the KLM at $n=0.9, J / W=0.2$ with a two-dimensional square-lattice DOS obtained via DMFT is shown.

One observes a hybridized bandstructure, fairly broadened by finite imaginary parts of the self-energy. Right at the Fermi energy $\omega=0$, a full gap in the flat band of heavy quasiparticles can be observed. Actually, the flat part of the lower band splits into particle and hole components as in the structures obtained in a static MF description, cf. (Fig. 5.1). The flat structures are rather sharp and thus resemble quasiparticles of a well-defined heavy Fermi liquid. At frequencies $\omega \approx \pm 0.15$ the splitting between flat and strongly dispersive parts is visible. For positive frequencies the gap $\Delta_{>}$is almost well developed, while for negative frequencies it is almost completely smeared out except for a slightly visible lack of spectral weight.

The mean-field description thus provides a good starting point to interpret the spectral structures arising in DMFT results of the KLM, not only in the paramagnetic, but also in the superconducting phase. Especially the splitting into a four-fold bandstructure observed in the static MF description helps to clearly identify the blurred quasiparticle bands in the DMFT-spectra. 


\subsection{Superconductivity at Zero Temperature}

At zero temperature, stable $s$-wave superconducting results over a large region of the $(n, J)$-phase diagram are obtained within the DMFT $+\mathrm{NRG}$ approach. Its properties are discussed in the following.

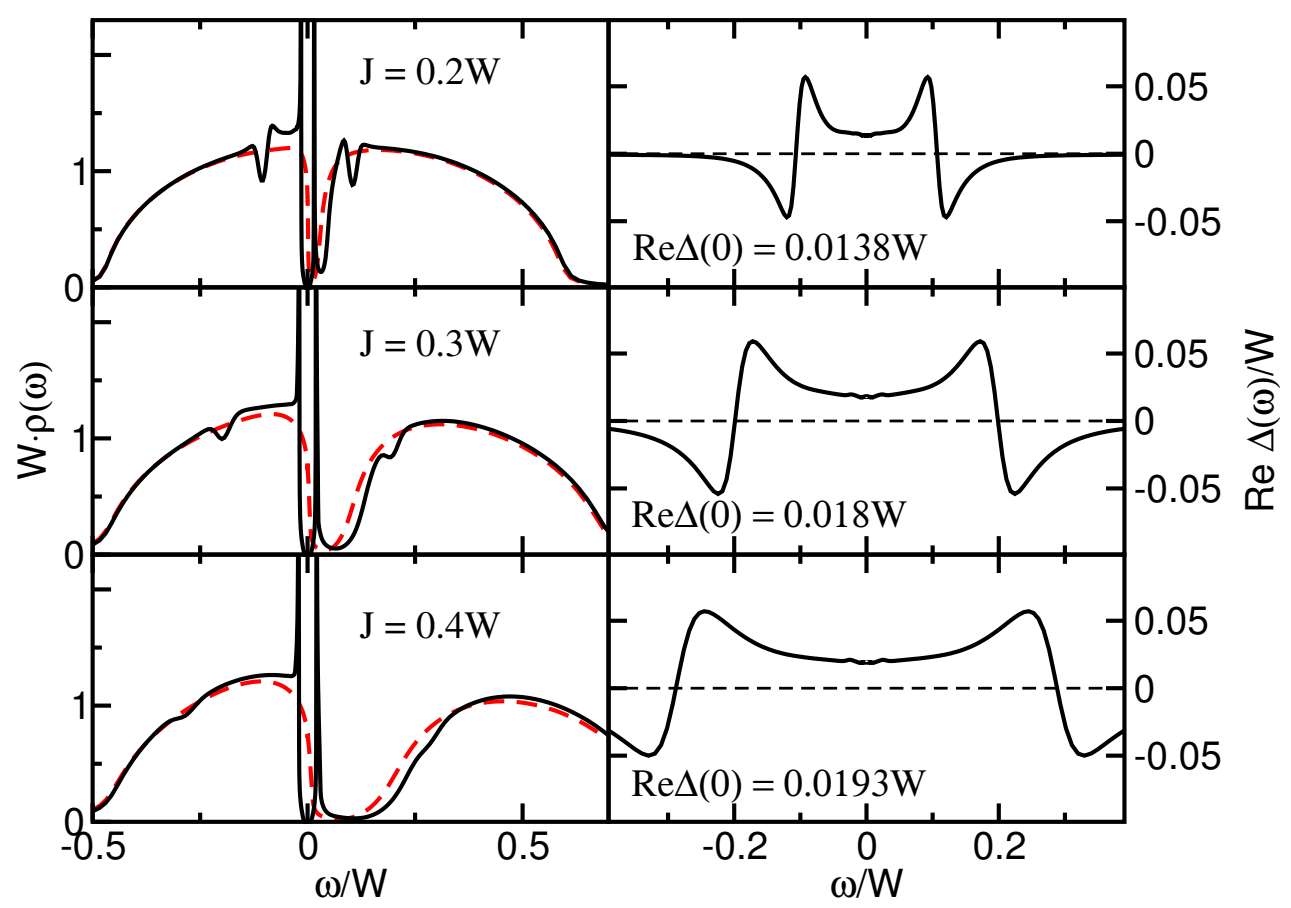

Figure 5.3: (Published in Ref. [Bod13]) Left panel: N DOS (red, dashed) and SC DOS (black) for $n=0.9$ and several $J$. Right panel: Real part of the frequency-dependent gap function. Note the different $\omega$-scales.

In (Fig. 5.3) the conduction band density of states in both the paramagnetic normal $(\mathrm{N})$ and superconducting (SC) phase are shown for different Kondo couplings $J$ at a fixed conduction band filling $n=0.9$. The only feature in the N DOS is a hybridization pseudo-gap above the Fermi energy which signals the formation of heavy quasiparticles. This formation can be rationalized within the picture of hybridized $c$ - and $f$-bands as discussed in section 3.1 .2 already.

The SC DOS is richer in structure since two additional features appear in the SC phase. First, a true BCS-like superconducting gap is present around the Fermi energy. It is limited by well-developed singularities and grows with increasing $J$ in the range of Kondo couplings shown in the figure. Secondly, side-resonances are visible in the DOS at positions which roughly scale with $J$. These structures are to a certain degree similar to the resonance observed in the AF DOS, though somewhat less distinct. Interestingly, these resonances 
are the most pronounced in the same parameter region as the spin resonances are in the AF case. In connection with superconductivity the appearance of side- or spin-resonances in the DOS is usually observed in strong-coupling superconductivity [Sca66].

To conclusively identify the solution as superconducting, the appearance of a gap alone is not sufficient, but rather the off-diagonal observables and dynamical quantities need to be studied. One is the anomalous part of the self-energy, a finite value of which clearly identifies the system as superconducting. It enters the definition of a frequency-dependent superconducting gap function, usually used in Eliashberg theory:

$$
\Delta(\omega)=\frac{\Sigma_{1}(\omega)+i \Sigma_{2}(\omega)}{1-\Sigma_{0} / \omega} .
$$

The $\Sigma_{i}(\omega)$ are the components of the self-energy matrix expanded in Pauli matrices, $\Sigma(\omega)=\sum_{\alpha} \Sigma_{\alpha}(\omega) \boldsymbol{\tau}_{\alpha}, \alpha=0,1,2,3$, where $\boldsymbol{\tau}_{0}$ denotes the identity matrix.

The resulting real part $\operatorname{Re} \Delta(\omega)$ of the gap function is symmetric as it is expected for even-frequency superconductivity, see right panel of (Fig. 5.3). It is strongly frequency dependent and shows sharp features which are broadened and shifted to higher energies with increasing $J$. These peaks are locally point symmetric around the zeroes $\omega_{0}^{ \pm}$where $\operatorname{Re} \Delta(\omega)$ changes its sign. For frequencies higher than $\left|\omega_{0}\right|$ it decays to zero within a fraction of the bandwidth. Such a sign change of the gap function followed by a decay indicates that the effective interaction among the paired quasiparticles turns from attractive to repulsive. ${ }^{1}$ In the case of heavy fermions it furthermore hints to an attractive interaction present among the heavy quasiparticle band only; the resonance position roughly scales with $J$ and is located at energies closely above the pseudogap which resembles the quasiparticle bandwidth. This supports above argument since the zeroes of the gap function coincide with the minima of the side resonances - they mark the renormalized bandwidth of the heavy quasiparticles to which the pairing is restricted.

Even more support for this argument can be obtained from the energy-resolved spectral functions. In (Fig. 5.4) the SC spectral function $A\left(\varepsilon_{\mathbf{k}}, \omega\right)$ is plotted in comparison to the $\varepsilon$-integrated DOS $\rho(\omega)$ for $J / W=0.2$ and $J / W=0.3$. On a large scale, one observes a hybridized bandstructure, which is the more pronounced the larger $J$ is. For $J / W=0.2$ the diverse structure close to the gap becomes visible for energies $\omega / W<0.2$. As it has been observed above, the number of bands has doubled from two to four: Close to the Fermi energy two separated flat bands appear. The gap between the two upper bands and between the two lower bands, respectively, is smeared out by the self-energy and thus

1 Actually, a causal connection the other way around can be shown by means of a Bardeen-Pines interaction. It simply extends the Coulomb potential such that it is attractive for a finite frequency range around the Fermi energy, but repulsive otherwise. As a result, the corresponding gap function exhibits a sign change exactly at the point where the effective potential changes sign, too Col13. 

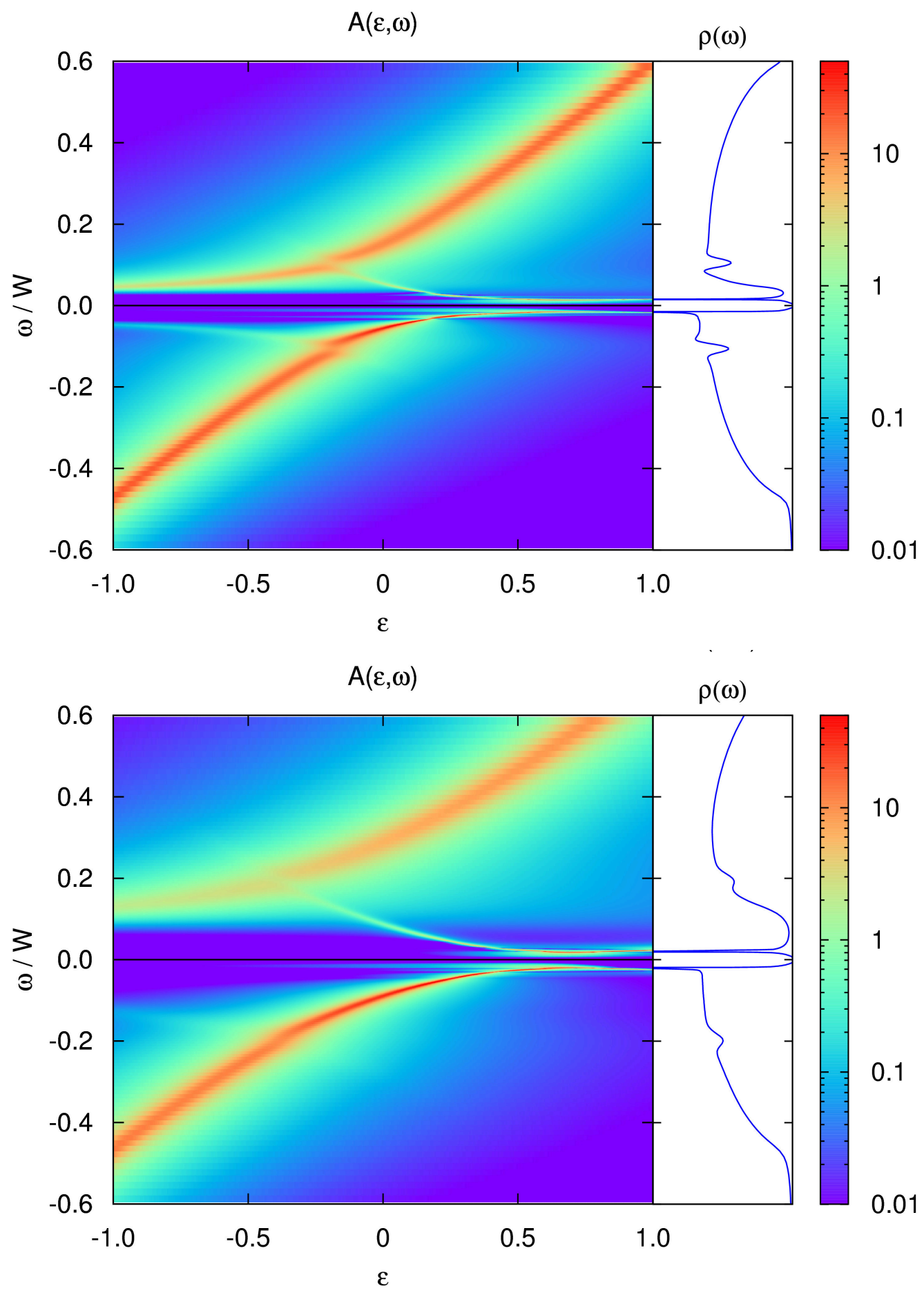

Figure 5.4: Spectral function $A\left(\varepsilon_{\mathbf{k}}, \omega\right)$ and corresponding DOS $\rho(\omega)$ for $J / W=0.2$ (top) and $J / W=0.3$ (bottom). Note that colors are scaled logarithmically as above. 
only a weak dip in spectral weight is visible. Importantly, the band splitting is located exactly where the side resonances appear in the DOS. ${ }^{1}$ Around $\varepsilon=0$, the spectral weight in the upper part of the $\omega<0$ band is rather high and the corresponding structure becomes increasingly sharp towards $\omega=0$, before it ends up in the singularities at the SC gap edge. It can be seen that the structures close to the Fermi energy are mirrored at the $\varepsilon$-axis to a certain degree, i.e., apart from different spectral weight these structures are particle-hole symmetric (note the logarithmic color scale). This feature in the single-particle spectrum is a result from weak Cooper pairing at energies higher than the gap edge.

It can be seen that the structures associated with superconductivity are limited to the flat heavy-quasiparticle bands. The latter are, in turn, restricted to energies $|\omega| \lesssim T_{0}$. For larger couplings such as $J / W=0.3$ in (Fig. 5.4) the band-splitting at the side-resonances is hardly visible in the spectral function anymore, since the larger self-energy contributions smear out the side gaps more and more. The above arguments are also consistent with the dispersion obtained in the static mean-field description, cf. (Fig. 5.1). In summary, it can be concluded that a hybridized bandstructure with well-formed heavy quasiparticles is a necessary prerequisite for the observed type of superconductivity in the KLM. Cooper pairing is, in fact, limited to the heavy Fermi liquid.

As an aside, but quite interestingly, even for half filling a stable superconducting solution is found in a small region $0.05<J / W<0.2$. Examples are shown in (Fig. 5.5). SC here emerges where the KLM is a Kondo insulator in the paramagnetic phase, which is particularly interesting. Furthermore the superconducting "dome" lies well inside the AF regime. For $J / W=0.15$ it can be nicely seen that the singularities at the gap edge are effectively suppressed. The corresponding anomalous expectation values $\Phi$ are, however, roughly an order of magnitude smaller compared to $n<1$. Numerical reliability can thus not be absolutely ensured.

\subsubsection{Static Properties and Zero-Temperature Phase Diagram}

The zero-frequency limit $\operatorname{Re} \Delta(0)$ provides an estimate for the gap seen in the DOS. It exhibits the same non-monotonic behavior as the true gap $\Delta_{\mathrm{sc}}$, which, as function of $J$, first increases up to $J / W \approx 0.5$ and then slowly decreases again, cf. (Fig. 5.3). This behavior is not necessarily the same as for the anomalous expectation value $\Phi=\left\langle\hat{c}_{i \uparrow} \hat{c}_{i \downarrow}\right\rangle$ [Xu 87]. As a static observable the latter nevertheless serves as a good simple quantity to explore the parameter region in which a stable superconducting solution exists. The evolution of $\Phi$ across the $(n, J)$-"phase diagram" is presented in (Fig. 5.6). At zero temperature a stable

1 It should be noted that the bandstructure is very similar to the half-filled case in the AF phase: Due to the doubling of the unit cell, the number of bands doubles as well. The position of the spin resonance there also coincides with the band splitting, cf. chapter 4 


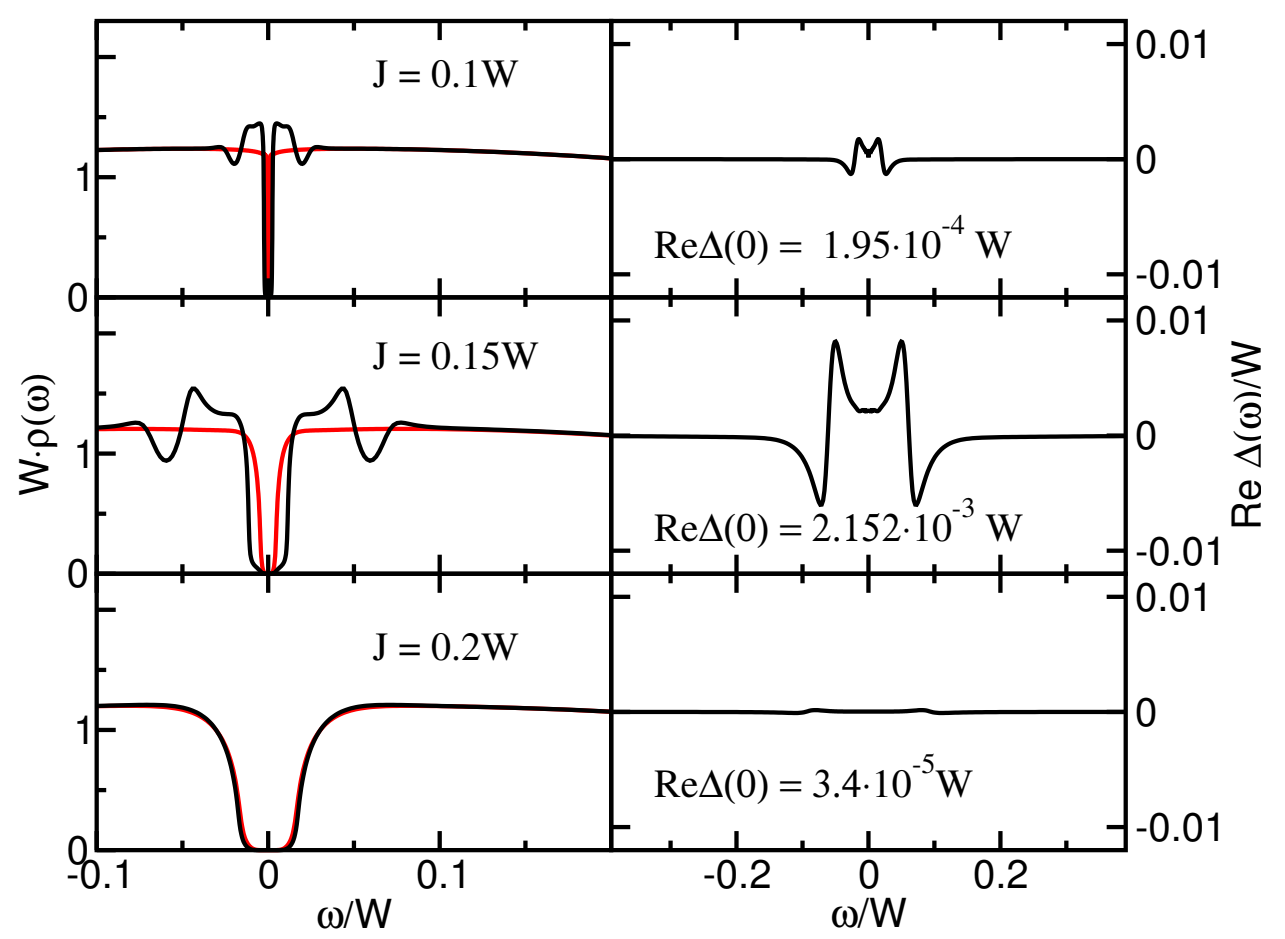

Figure 5.5: Left panel: N DOS (red) and SC DOS (black) for the KLM at half filling. Right panel: Real part of the corresponding gap functions.

superconducting phase can be found in a large region of parameters $(n, J)$. For all fillings between $n=0.5$ and $n \lesssim 1$ a lower threshold $J_{\min } / W=0.1$ exists, above which the pairing is stabilized. For lower values of $J$ no stable superconducting solution can be found as the anomalous quantities are that small that it is hardly possible to distinguish them from numerical noise. A maximum of $\Phi$ is found for $J \approx 0.25$ and $n=0.85$, opposed to $\operatorname{Re} \Delta(0)$ which increases up to $J / W \approx 0.5$ before it slowly decreases again for larger couplings. In region of the maximal $\Phi$, however, the side resonances are also the most pronounced. ${ }^{1}$ Focusing on a fixed $J / W=0.2$ a superconducting solution is found for fillings $0.45<n<1$. For larger $J / W$ a finite order parameter $\Phi$ extends to even lower fillings.

A more detailed evolution of $\Phi$ along two cuts in the phase diagram, indicated by white dashed lines in (Fig. 5.6), is shown in (Fig. 5.7). For a fixed filling $n=0.9$ the evolution of $\Phi$ is shown in the left panel. In addition, the evolution of the lattice coherence scale $T_{0}$ is shown. However, there seems to be no simple connection between $T_{0}$ and $\Phi$. For small

1 A similar connection of the resonances to $c$-electrons is observed in the AF case: There they are the most pronounced, where the magnetization of the conduction band - and not of the local moments - is the largest. 


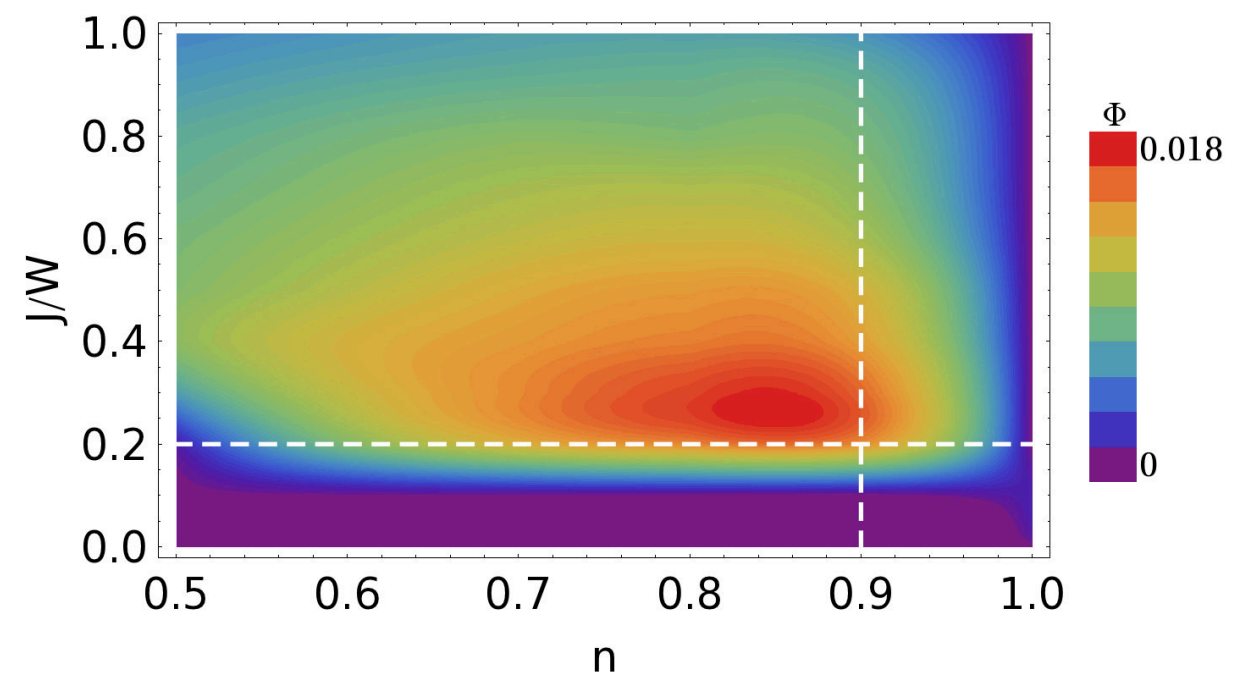

Figure 5.6: (Published in Ref. [Bod13]) Anomalous expectation value $\Phi$ as function of $J$ and $n$ between quarter and half filling.

couplings $\Phi(J)$ seems to scale with $T_{0}$. For strong couplings $J>W / 2$, on the other hand, the anomalous order parameter decays roughly as $1 / J$. The $n$-dependence of both $\Phi$ and $T_{0}$ is shown in the right panel of (Fig. 5.7) and does not reveal a simple dependence $\Phi\left(T_{0}\right)$ either.
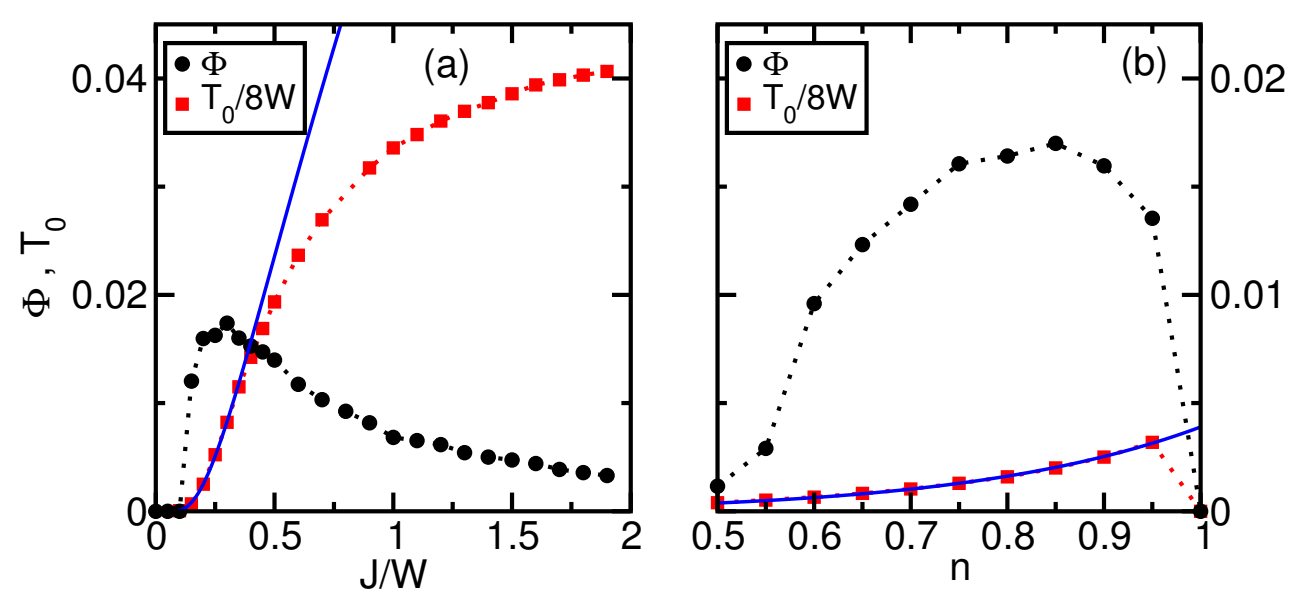

Figure 5.7: (Published in Ref. [Bod13]) Left panel (a): $\Phi$ and $T_{0}$ as function of $J$ at fixed $n=0.9$. Right panel (b): $\Phi$ and $T_{0}$ as function of $n$ at a fixed $J / W=0.2$. The full lines represent approximate dependencies of $T_{0}$ on $J$ and $n$, respectively (cf. Sec. 3.2.1). 


\section{Competition with Magnetism}

The superconducting order may compete with magnetically ordered states of the KLM. Within DMFT, both antiferromagnetism close to half filling and a ferromagnetic phase at small fillings are observed [Pet07, Ots09, Bod11]. These magnetic phases have, however, a limited extent in both $J$ and $n$. For example, at $n=0.9$ AF order is only found for $J<J_{c} \approx 0.25 W$ [Bod11, cf. chapter 4. FM order is only found for $n<n_{c} \approx 0.65$ [Pet07, San02. The region where a strong superconducting phase is found in (Fig. 5.6) is complementary to the regions with magnetic order, although the phase boundaries seem to overlap. The actual interplay or competition of AF, FM and $\mathrm{SC}$ in these regions is of high interest and still an open question.

\subsection{Finite-Temperature Behavior}

Finite temperature properties of the superconducting state in the KLM are deeply interesting, especially in order to obtain a critical temperature $T_{c}$ at which superconductivity completely vanishes. For accurate results at $T>0$ within the NRG it is necessary to use the full-density-matrix (FDM) approach [Wei07] in combination with $z$-averaging [Ž09b]. In (Fig. 5.8) the temperature evolution of the SC DOS is shown for $n=0.9$ and $J / W=0.25$, where SC is rather pronounced, cf. (Fig. 5.6). With increasing temperature $T$ the SC gap shrinks and the side resonances are depleted. Both observations reveal reduced pair correlations. This is even more obvious by inspection of $\Phi(T)$, shown in the inset. Over a wide range up to $T / W, \Phi$ stays almost constant, while a further temperature increase initiates a decrease in $\Phi$. Close to $T_{c}$ the anomalous expectation value finally drops down with a rather steep slope. In this region the gap is also progressively filled and the singularities at the gap edge get more and more depleted. Both the hybridization gap and the side resonance move towards the Fermi level as $T \rightarrow T_{c}$. Finally, for $T>T_{c}$, where the system is in the normal paramagnetic state, only the hybridization gap is visible. This is again a hint for the essential prerequisite of a HF state for local superconducting order in the KLM, since it vanishes before the lattice Kondo-effect does.

The exact determination of $T_{c}$ from the numerical data is challenging, since close to $T_{c}$ the signatures of superconductivity become as small as numerical noise. Also, the convergence close to phase transitions is generally rather slow in DMFT due to critical slowing down [Ž09a]. From the data in (Fig. 5.8) a $T_{c}=0.0036 \mathrm{~W}$ is extracted at $n=0.9$ and $J / W=0.25$. This is well below the corresponding $T_{0}$. More results at a fixed $n=0.9$ are collected in Tab. 5.1 for several values $J$ in the region where optimal conditions for pairing seem to be fulfilled, cf. (Fig. 5.7). It is generally observed that $T_{c}<T_{0}$, i.e., the $\mathrm{HF}$ state is necessary for the emergence of SC. This leads to a strong suppression of $T_{c}$ for 


\begin{tabular}{l|l|l|l|l|l}
\hline$J / W$ & 0.2 & 0.25 & 0.3 & 0.4 & 0.5 \\
\hline$T_{0} / W$ & 0.0200 & 0.0418 & 0.0658 & 0.1139 & 0.1548 \\
\hline$T_{c} / W$ & 0.0027 & 0.0036 & 0.0054 & 0.0058 & 0.0054 \\
\hline$\Phi(T=0)$ & 0.0160 & 0.0163 & 0.0174 & 0.0153 & 0.0140 \\
\hline $\operatorname{Re} \Delta(0) / W$ & 0.0138 & 0.0165 & 0.0180 & 0.0193 & 0.0195 \\
\hline \hline $\operatorname{Re} \Delta(0) / T_{c}$ & 5.169 & 4.583 & 3.321 & 3.305 & 3.585 \\
\hline
\end{tabular}

Table 5.1: Quantities characterizing the superconducting solution for $n=0.9$ as a function of $J$ in the region of optimal pairing conditions.

small $J$. On the contrary, the SC gap Re $\Delta(0)$ is less sensitive to both $J$ and $T_{0}$ in this regime: From $J / W=0.5$ to 0.2 the gap changes only by roughly $15 \%$ while $\Phi$ is reduced to half of its initial value. Another interesting quantity is the ratio $\operatorname{Re} \Delta(0) / T_{c} \equiv \Delta / T_{c}$. In Tab. 5.1 these results are shown in the last row. The BCS-ratio of $\Delta / T_{c} \approx 1.74$ is exceeded by a considerable factor between 2 and 3. Such values are actually observed in HF superconductors [Sto11, Miz11], although there these values are interpreted by means of a weak-coupling theory for $d$-wave pairing.

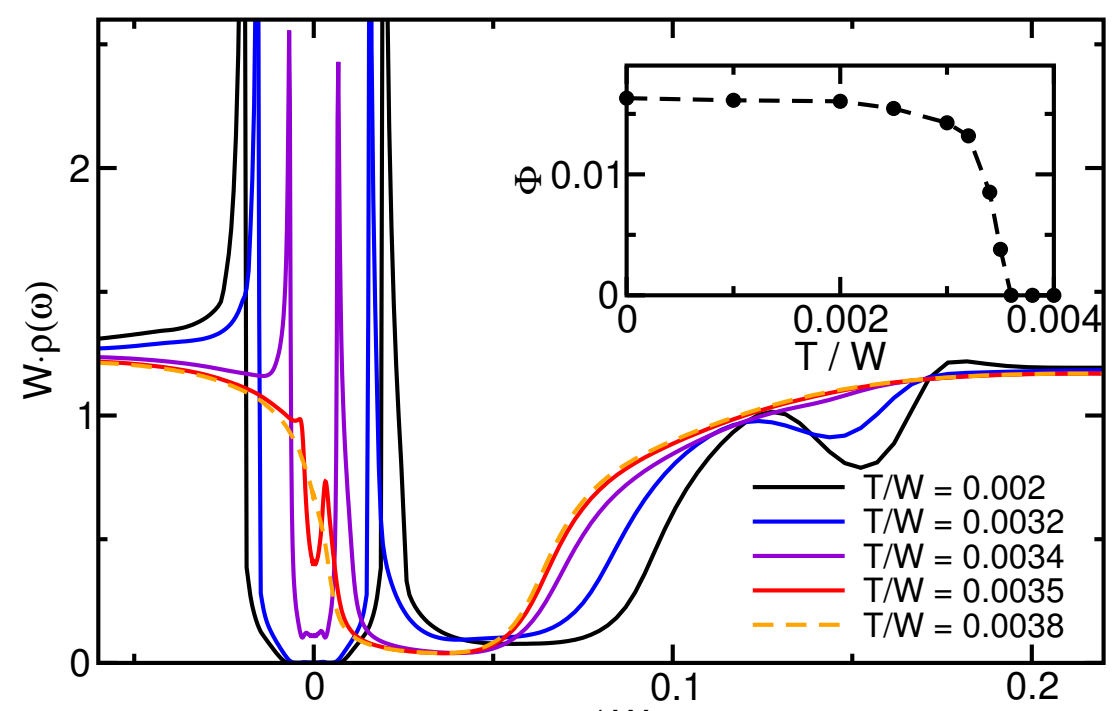

$\omega / \mathrm{W}$

Figure 5.8: Evolution of the SC DOS with increasing temperature. Coherence scale $T_{0} / W=0.0105$ at $T \rightarrow 0$ for the fixed parameters $n=0.9, J / W=0.25$. Inset: Evolution of $\Phi$ with temperature. For $T>T_{c} \approx 0.0036 \mathrm{~W}$ pair correlations are fully suppressed. 


\subsection{Pairing Mechanism}

The behavior of pair correlations expressed by $\Phi(J)$ and the pairing mechanism can, at least in the strong coupling regime $J>W$, be understood perturbatively, cf. (Fig. 5.9). Therefore, a conduction-band filling $n \approx N_{\mathrm{el}} / N_{s} \lesssim 1$ of $N_{\mathrm{el}}$ electrons on $N_{s}$ sites is considered. In the strong coupling limit $J / W \rightarrow \infty$ there are $N_{\text {el }}$ Kondo singlets and $N_{s}-N_{\text {el }}$ unscreened local moments. Since the conduction electrons are itinerant, these unscreened local moments can also be interpreted as spinful $c$-holes with a density $(1-n)$ and a hard-core repulsion. They form a Fermi liquid with a corresponding coherence scale $T_{0} \propto W$ in the strong coupling limit ${ }^{1}$. For large but finite Kondo couplings virtual excitations are allowed - the lowest one converts a singlet into a triplet a can be created by hopping in the conduction band (cf. Fig. 5.9): If a spin-up electron of the right-hand singlet tunnels to the intermediate site, a triplet state is virtually excited there. The excited triplet can then decay via another neighboring hole - provided that its spin is opposite to the other one. Antiferromagnetically ordered local $(f-)$ moments do thus support this mechanism. Altogether, this virtual second-order process leads to correlated hopping of holes with an energy gain $\propto W^{2} / J$. It binds two holes into a singlet state, cf. (Fig. 5.9). The pairing occurs locally on the intermediate site, which is only shared by the holes in the virtual triplet state. Hence, the local pairing is strongly retarded. After the two hopping processes, the spin-down hole has moved by two sites from the left to the right across a spin-up hole by exciting an intermediate triplet state.

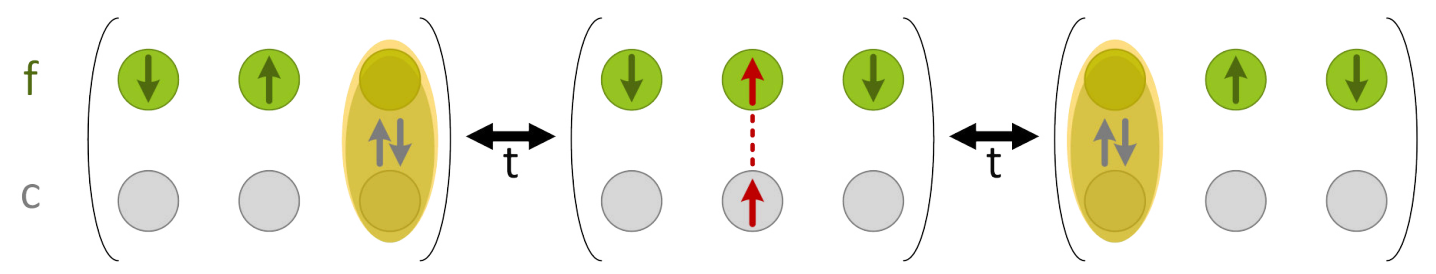

Figure 5.9: Second-order hopping process responsible for pairing in the strong coupling limit $J>W$. The $c$ electron and local moment $(f-)$ configurations on adjacent lattice sites are depicted. The yellow ellipse represents a singlet of two electrons, the intermediate triplet state is indicated by red arrows.

This pairing mechanism requires Kondo screening to be intact since it allows for the existence of the virtual triplet state. Thus, it also applies to smaller $J$. The energy of the virtual state may be approximated by the Kondo binding energy or coherence scale $T_{0}$, which directly explains the limiting fact $T_{c}<T_{0}$ in the KLM. Moreover, in this picture it becomes plausible that SC is more favorable close to half filling. At $n \lesssim 1$ Kondo screening

1 In turn, the impurity Kondo scale diverges proportional to $J$. 
involves conduction electrons while the Cooper pairs are comprised of $c$-holes. In the "exhaustion limit" of small fillings, Kondo screening processes become less $-T_{0}$ falls off exponentially away from $n=1$ - whereby also the second-order processes responsible for pairing are less frequent.

\subsection{Eliashberg Theory for Spin-Fermion Models}

In this section the approach of magnetic Eliashberg equations including spin fluctuations [Nor87, Nor88, Pla06] is applied to the KLM. It brings up another argument why local spin fluctuations can serve as a "pairing glue" and drive superconductivity for the KLM in DMFT. The section closely follows Ref. [Pla06] but is adapted to the KLM. It is started with a general model that captures the interaction of electrons and spin fluctuations,

$$
H=\sum_{\mathbf{k} \sigma} \varepsilon_{\mathbf{k} \sigma} c_{\mathbf{k} \sigma}^{\dagger} c_{\mathbf{k} \sigma}+\sum_{\mathbf{k}, \sigma, \sigma^{\prime}} \underbrace{\left(V_{\mathrm{sf}}(\mathbf{k}) \sum_{\alpha=x, y, z} S_{\mathbf{k}}^{\alpha} \boldsymbol{\tau}_{\sigma \sigma^{\prime}}^{\alpha}\right)}_{=: W_{\sigma \sigma^{\prime}}^{\mathrm{sf}}(\mathbf{k})} c_{\mathbf{k} \sigma}^{\dagger} c_{\mathbf{k} \sigma^{\prime}} .
$$

The Pauli spin matrices are denoted by $\boldsymbol{\tau}_{\sigma \sigma^{\prime}}^{\alpha}(\alpha=x, y, z)$ and a local Kondo interaction $V_{\mathrm{sf}}(\mathbf{k}) \equiv J$ is assumed throughout this work. The second term can be written as

$$
H_{\mathrm{sf}}=J \sum_{\mathbf{k}}\left(S_{\mathbf{k}}^{z}\left(c_{\mathbf{k} \uparrow}^{\dagger} c_{\mathbf{k} \uparrow}-c_{\mathbf{k} \downarrow}^{\dagger} c_{\mathbf{k} \downarrow}\right) / 2+S_{\mathbf{k}}^{+} c_{\mathbf{k} \downarrow}^{\dagger} c_{\mathbf{k} \uparrow}+S_{\mathbf{k}}^{-} c_{\mathbf{k} \uparrow}^{\dagger} c_{\mathbf{k} \downarrow}\right)
$$

In order to account for superconductivity, the Nambu formalism for the corresponding Green's functions needs to be employed again. With the Nambu spinors $\Psi_{\mathbf{k} \sigma}(t)$ in the Heisenberg presentation, the first equation of motion can be derived as

$$
\left(\omega \mathbf{1}-\varepsilon_{\mathbf{k} \sigma} \boldsymbol{\tau}_{3}\right) \mathbf{G}_{\mathbf{k}, \sigma}(\omega)=\mathbf{1}_{2}+\sum_{\sigma^{\prime}}\left\langle\left\langle W_{\sigma \sigma^{\prime}}^{\mathrm{sf}}(\mathbf{k}) \Psi_{\mathbf{k} \sigma^{\prime}} ; \Psi_{\mathbf{k} \sigma}^{\dagger}\right\rangle\right\rangle_{\omega}
$$

which is equivalent to the Dyson equation in the form

$$
\left[\mathbf{G}_{\mathbf{k}, \sigma}^{(0)}(\omega)\right]^{-1} \mathbf{G}_{\mathbf{k}, \sigma}(\omega)=\mathbf{1}_{2}+\Sigma_{\mathbf{k}, \sigma}(\omega) \mathbf{G}_{\mathbf{k}, \sigma}(\omega)
$$

Comparing the former two equations, one obtains the self-energy as

$$
\mathbf{\Sigma}_{\mathbf{k}, \sigma}(\omega)=\sum_{\sigma^{\prime}}\left\langle\left\langle W_{\sigma \sigma^{\prime}}^{\mathrm{sf}}(\mathbf{k}) \Psi_{\mathbf{k} \sigma^{\prime}} ; \Psi_{\mathbf{k} \sigma}^{\dagger}\right\rangle\right\rangle_{\omega} \cdot\left[\mathbf{G}_{\mathbf{k}, \sigma}(\omega)\right]^{-1}
$$

which resembles the self-energy trick used in NRG, though in a k-dependent form here. The higher Green's function in (5.4) can now be differentiated over the second time argument 
$t^{\prime}$ such that a second equation of motion is obtained:

$$
\left\langle\left\langle W_{\sigma \sigma^{\prime}}^{\mathrm{sf}}(\mathbf{k}) \Psi_{\mathbf{k}, \sigma^{\prime}} ; \Psi_{\mathbf{k}, \sigma}^{\dagger}\right\rangle\right\rangle_{\omega}=\left\langle\left\langle W_{\sigma \sigma^{\prime}}^{\mathrm{sf}}(\mathbf{k}) \Psi_{\mathbf{k}, \sigma^{\prime}} ; \sum_{\sigma^{\prime \prime}} \Psi_{\mathbf{k}, \sigma^{\prime \prime}}^{\dagger}\left(W_{\sigma \sigma^{\prime \prime}}^{\mathrm{sf}}(\mathbf{k})\right)^{\dagger}\right\rangle\right\rangle_{\omega} \mathbf{G}_{\mathbf{k}, \sigma}^{(0)}(\omega)
$$

It is assumed that no spin-ordering is present and hence the average $\left\langle W_{\sigma \sigma^{\prime}}^{\mathrm{sf}}(\mathbf{k})\right\rangle=0$ vanishes. The self-energy can now be related to the irreducible part of the scattering matrix [Pla06],

$$
\boldsymbol{\Sigma}_{\mathbf{k}, \sigma}(\omega)=\sum_{\sigma^{\prime}, \sigma^{\prime \prime}}\left\langle\left\langle W_{\sigma \sigma^{\prime}}^{\mathrm{sf}}(\mathbf{k}) \Psi_{\mathbf{k} \sigma^{\prime}} ; \Psi_{\mathbf{k} \sigma^{\prime \prime}}^{\dagger}\left(W_{\sigma \sigma^{\prime \prime}}^{\mathrm{sf}}(\mathbf{k})\right)^{\dagger}\right\rangle\right\rangle_{\omega}^{\mathrm{irr}} .
$$

It can be used to derive an approximate form of the self-energy, the off-diagonal part of which is needed to derive a gap equation. The non-crossing approximation (NCA) Gre83, Kur83 yields a beneficial simplification. Within NCA, the propagation of fermionic and bosonic excitations are assumed to be independent of each other, such that the time-dependent correlation function can be decoupled:

$$
\begin{aligned}
& \left\langle W_{\sigma \sigma^{\prime}}^{\mathrm{sf}}(\mathbf{k}, t) \Psi_{\mathbf{k} \sigma^{\prime}}(t) ; \Psi_{\mathbf{k} \sigma^{\prime \prime}}^{\dagger}\left(W_{\sigma \sigma^{\prime \prime}}^{\mathrm{sf}}(\mathbf{k})\right)^{\dagger}\right\rangle \\
& \approx\left\langle W_{\sigma \sigma^{\prime}}^{\mathrm{sf}}(\mathbf{k}, t) ;\left(W_{\sigma \sigma^{\prime}}^{\mathrm{sf}}(\mathbf{k})\right)^{\dagger}\right\rangle\left\langle\Psi_{\mathbf{k} \sigma^{\prime}}(t) ; \Psi_{\mathbf{k} \sigma^{\prime \prime}}^{\dagger}\right\rangle \\
& =\delta_{\sigma^{\prime} \sigma^{\prime \prime}}|J|^{2} \sum_{\alpha}\left\langle S_{\mathbf{k}}^{\alpha}(t) ; S_{\mathbf{k}}^{\dagger \alpha}\right\rangle \boldsymbol{\tau}_{\sigma \sigma^{\prime}}^{\alpha} \boldsymbol{\tau}_{\sigma^{\prime \prime} \sigma}^{\alpha}\left\langle\Psi_{\mathbf{k} \sigma^{\prime}}(t) ; \Psi_{\mathbf{k} \sigma^{\prime \prime}}^{\dagger}\right\rangle
\end{aligned}
$$

The time-dependent correlation functions can be represented as

$$
\left\langle A_{p}(t) ; A_{p}^{\dagger}\right\rangle=\int_{-\infty}^{\infty} \mathrm{d} \omega \frac{\mathrm{e}^{-i \omega t}}{1 \pm \mathrm{e}^{-\beta \omega}}\left(-\frac{1}{\pi} \operatorname{Im}\left\langle\left\langle A_{p} ; A_{p}^{\dagger}\right\rangle\right\rangle_{\omega+i 0^{+}}\right),
$$

such that the self-energy can be written as

$$
\Sigma_{\mathbf{k}, \sigma}(\omega)=\int_{-\infty}^{\infty} \mathrm{d} z K(\omega, z ; \mathbf{k})\left(-\frac{1}{\pi} \operatorname{Im} \boldsymbol{G}_{\mathbf{k} \sigma}(z)\right)
$$

The Kernel of the integral equation Eq. (5.8) has the same form as in Eliashberg theory Pla06,

$$
K(\omega, z ; \mathbf{k})=\int_{-\infty}^{\infty} \mathrm{d} \Omega \frac{\tanh z / 2 T+\operatorname{coth} \Omega / 2 T}{2(\omega-z-\Omega)} \lambda(\mathbf{k}, \Omega)
$$

with the spin-mediated electron-electron interaction

$$
\lambda(\mathbf{q}, \omega)=|J|^{2}\left(-\frac{3}{\pi} \operatorname{Im}\left\langle\left\langle S_{\mathbf{q}}^{z} ; S_{-\mathbf{q}}^{z}\right\rangle\right)\right)=\frac{3|J|^{2}}{\pi} \operatorname{Im} \chi_{\mathbf{q}}^{z z}(\omega) .
$$


It is assumed that no magnetic order is present so that the spin susceptibility $\chi_{\mathbf{q}}^{ \pm}(\omega)=$ $2 \chi_{\mathbf{q}}^{z z}(\omega)=-2\left\langle\left\langle S_{\mathbf{q}}^{z} ; S_{-\mathbf{q}}^{z}\right\rangle\right\rangle_{\omega}$ is isotropic. Note that the spin susceptibility corresponds to the local $(f-)$ moments.

In a local DMFT approximation all momentum dependencies in the Eliashberg-type Eq. (5.8) can be neglected, since one deals with an effective single-site problem. Moreover, assuming that the system constitutes a local Fermi liquid with renormalized parameters, Hewson's renormalized perturbation theory [Hew93a] can be employed. As the dynamic Kondo spin is a dynamic quantity with a fluctuation spectrum with a support on the order of the Kondo scale, the usual approximations in Eliashberg theory cannot be used: The relevant energy scales are of order $T_{0}$ and must not be put to the Fermi energy.

The off-diagonal component of Eq. (5.8) in the local approximation reads

$$
\Sigma_{\sigma}^{(12)}\left(\omega+i 0^{+}\right)=\int_{-\infty}^{\infty} \mathrm{d} \omega^{\prime} K\left(\omega, \omega^{\prime}\right)\left(-\frac{1}{\pi} \operatorname{lm} G^{(12)}\left(\omega^{\prime}+i 0^{+}\right)\right)
$$

with the kernel from Eq. (5.9) in a k-independent form. Eq. (5.10) constitutes the gap equation which needs to be solved.

In Eliashberg theory with phonons the common approximation at this point is $\omega, \omega^{\prime} \ll$ $\Omega \ll \mu$ and hence $\omega \approx \omega^{\prime} \approx 0$, leading in the spin-fluctuation case to

$$
K\left(\omega, \omega^{\prime}\right) \approx-\frac{3|J|^{2}}{2} \tanh \left(\omega^{\prime} / 2 T\right) \underbrace{\int_{-\infty}^{\infty} \frac{\mathrm{d} \Omega}{\Omega}\left(\frac{1}{\pi} \operatorname{Im} \chi^{z z}(\Omega)\right)}_{\operatorname{Re} \chi^{z z}(0)} .
$$

The positive contribution of $\operatorname{Re}^{z z} \chi(0)>0$ yields a negative and hence repulsive kernel which will not yield an $s$-wave even-frequency solution. In HF systems, however, the above approximations are not valid any longer: For the Fermi liquid the chemical potential shifts $\mu \longrightarrow \tilde{\mu} \sim T_{0}$ and therefore one may not assume $\omega, \omega^{\prime} \ll \mu$.

Assuming the system to be deep within the Fermi liquid phase, temperature dependencies of Fermi-liquid parameters are negligible. For a frequency-independent gap one can put $\omega=0$ and retains the frequency dependence of $\chi$ in the kernel,

$$
\begin{aligned}
K\left(0, \omega^{\prime}\right) & \approx \frac{3|J|^{2}}{2} \tanh \left(\omega^{\prime} / 2 T\right) \int_{-\infty}^{\infty} \mathrm{d} \Omega \frac{\operatorname{Im} \chi^{z z}(\Omega)}{-\omega^{\prime}-\Omega} \\
& =-\frac{3|J|^{2}}{2} \tanh \left(\omega^{\prime} / 2 T\right) \operatorname{Re} \chi^{z z}\left(-\omega^{\prime}\right)
\end{aligned}
$$


where $\operatorname{Re} \chi^{z z}\left(-\omega^{\prime}\right)=\operatorname{Re} \chi^{z z}\left(\omega^{\prime}\right)$. The full spin-fluctuation spectrum of the Kondo spin is thus of high importance to the question whether superconductivity may arise or not.

In order to determine $T_{c}$ from Eq. (5.10), it is necessary to determine

$$
G^{(12)}(z)=\frac{\Sigma^{(12)}(z)}{\left(z+\varepsilon_{\mathbf{k}}-\Sigma^{(22)}(z)\right)\left(z-\varepsilon_{\mathbf{k}}-\Sigma^{(11)}(z)\right)-\left(\Sigma^{(12)}(z)\right)^{2}},
$$

where the the off-diagonal self-energy can, for a determination of $T_{c}$, be approximated by a step function in the numerator, $\Sigma^{(12)}\left(\omega+i 0^{+}\right) \approx \Delta \Theta(J / 2-\omega) \in \mathbb{R}$, and be set to zero in the denominator [Bod13. The diagonal self-energies are replaced by the approximations for a local Fermi liquid Hew93a, i.e., $\Sigma^{(11),(22)}(z)=\tilde{V}^{2} /(z+\tilde{\mu})$, where both the effective hybridization $\tilde{V}$ and $\tilde{\mu}$ are of order $T_{0}$. Further assuming $\tilde{\mu}=0$ on can perform the $\mathbf{k}$-sum to obtain the local off-diagonal Green's function,

$$
G^{(12)}(z)=i \pi \Delta \rho_{0}\left(z-\tilde{V}^{2} / z\right)^{-1}
$$

where a flat DOS $\rho_{0}$ of the conduction band and the wide-band limit is assumed. Putting together all the above approximations in the gap equation, one obtains

$$
1=\rho_{0} \int_{0}^{J / 2} \mathrm{~d} \omega K(0, \omega)\left(\frac{1}{\omega-\tilde{V}}+\frac{1}{\omega+\tilde{V}}\right)
$$

as equation to determine the critical temperature $T_{c}$.

The kernel contains the real part of the spin-susceptibility, which is shown in (Fig. 5.10). The kernel can be identified as negative for small $\omega$, cf. Eq. (5.12), and would thus be considered repulsive. However, the contribution from the off-diagonal Green's function within the approximation of a local Fermi liquid stemming from the Kondo effect is also negative in the region $|\omega| \lesssim c_{0} \cdot T_{0}, c_{0}>1$. The total contribution from the interval $\omega \in\left[0, T_{0}\right]$ is thus positive. An important constraint arises from the pole at $\omega=\tilde{V}$ : The sign change for $\omega>\tilde{V}$ requires $\operatorname{Re} \chi^{z z}(\omega)$ to decay rapidly on a scale $T_{0}$.

Taking the numerical data for $\operatorname{Re} \chi^{z z}(\omega)$ from (Fig. 5.10 ) and varying $\tilde{V}$ from $\tilde{V} \ll T_{0}$ to $\tilde{V} \gg T_{0}$ the integral value in Eq. (5.13) can be determined and the result is shown in the inset of (Fig. 5.10). In both limits the integral is negative and no solution exists. However, in the intermediate regime a reasonably large region with $\tilde{V}=\mathcal{O}\left(T_{0}\right)$ is present: A positive value of the integral yields a non-trivial solution and thus the tendency towards pairing.

The analysis identifies two absolutely necessary requirements for SC in the KLM: welldefined heavy quasiparticles with a small energy scale $T_{0}$, i.e. hybridized bands, and a spin fluctuation spectrum that decays on the same scale. Moreover, the above result predicts a strongly reduced $T_{c}$ for phonon-mediated HF superconductivity; the kernel there enters 


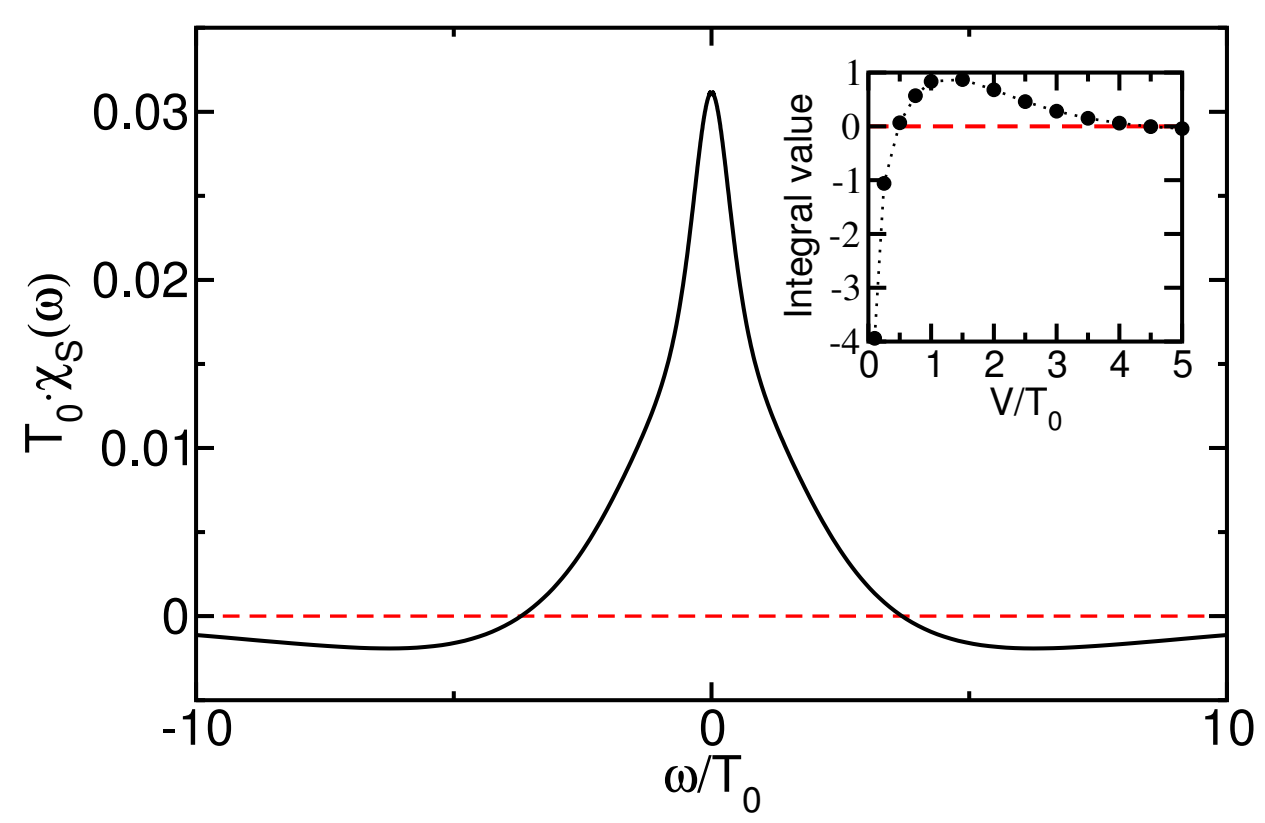

Figure 5.10: $\operatorname{Re} \chi^{z z}(\omega) \equiv \chi_{s}(\omega)$ in the PM phase of the KLM for $J / W=0.2$ and $n=$ 0.9. The red dashed line marks the zero. Inset: Value of the integral from Eq. (5.13) as function of $\tilde{V}$ at $T=0$.

with a negative sign and thus the low-energy part of the integral tends to suppress pairing instead.

Due to the uncontrolled approximations above, the discussion yields only a qualitative foundation for $s$-wave superconductivity in the KLM. Nevertheless, the result is contrary to common knowledge: Under the very special conditions met in heavy-fermion systems, spin fluctuations can lead to $s$-wave superconductivity.

\subsection{Summary}

In this chapter a novel mechanism for superconductivity in heavy-fermion materials has been identified; due to the Kondo exchange coupling, local spin fluctuations arise and can act as retarded "glue" for $s$-wave pairing of heavy quasiparticles. At energies related to the spin fluctuation spectrum, side resonances have been observed in the density of states, similar to the antiferromagnetic phase. By means of a static mean-field description these DMFT results have been interpreted as stemming from the separation between the 
flat quasiparticle bands close to the Fermi energy and the strongly dispersive part of the bandstructure. The correlation between the side-resonance position and the effective quasiparticle bandwidth has thus led to the conclusion that the superconducting order appears among heavy quasiparticles only. Further DMFT results shown in this chapter are, in fact, consistent with this conclusion. First, the observed gap function indicates a sign change of the effective interaction exactly at the resonance position. Secondly, finite temperature results revealed the general rule that $T_{c}<T_{0}$, i.e. the heavy-fermion state needs to be developed before superconductivity can appear in the HF liquid. Finally, both the proposed pairing mechanism and an Eliashberg-type analysis support the picture of low-energy local spin fluctuations gluing heavy quasiparticles together to heavy Cooper pairs. 



\section{Extensions of the Kondo-Lattice Model: Superconducting Properties}

The simple Kondo-lattice model is an extremely simplified minimal model for HF systems. Nevertheless, it captures the essential low-energy properties of HF systems and exhibits rather rich physics in ordered phases as presented in the previous chapters. It is thus a good basis to investigate basic mechanisms being responsible for antiferromagnetism or superconductivity. However, the complexity of real HF superconductors requires the inclusion of additional interactions, such as a Coulomb repulsion among conduction electrons or the coupling to phonon modes. In section 3.2 .2 it has already been shown how such additional interactions influence the low-energy scale. An on-site Coulomb repulsion enhances the coherence scale $T_{0}$ while the attractive interaction mediated by a local phonon mode strongly suppresses this scale. In the previous chapter it has been shown that the scale $T_{0}$ is closely related to superconductivity and that a well-developed HF state is a prerequisite for the Cooper pairs. One can thus expect a rather strong influence of these additional interactions on the presence and strength of a superconducting condensate. Another line of research aims at uranium-based HF superconductors, where the KLM with a higher local moment, $S=1$, provides a minimal model to describe the physics of the $5 f^{2}$ configuration of uranium ions dV91, Sto12].

In this chapter, the influence of the aforementioned interactions on the superconducting state in the KLM is discussed. Due to the large parameter spaces only some prototypical results are shown. First, a local Coulomb repulsion among the conduction electrons is taken into account, as discussed for the paramagnetic state in Sec. 3.2.2. In the same way, the inclusion of an Einstein-phonon mode locally coupled to the conduction electron density is investigated in the next section. At the end of this chapter, results for the superconducting phase of the KLM with a spin $S=1$ are presented. 


\subsection{Stabilized Pairing in the Correlated Kondo-Lattice Model}

The influence of an additional on-site repulsion $U$ on the paramagnetic state has already been discussed in section 3.2.2. The quasiparticle residue $Z$ and coherence scale $T_{0}$ increase monotonically with $U$, reflected in a growing pseudogap width. As shown in the last chapter, the superconducting state depends sensitively on $T_{0}$. A superconducting state only emerges above a minimal value of $T_{0}$ and the pair correlations are the most pronounced in a certain order of magnitude of $T_{0}$. The local on-site pairing is expected to be suppressed by a local Coulomb repulsion.

However, as the unconventional superconducting state in the KLM originates from local spin fluctuations, its interplay with a local repulsion is more subtle than a simple suppression. The dependence of the anomalous expectation value $\Phi=\left\langle c_{\uparrow} c_{\downarrow}\right\rangle$ and the gapwidth $\Delta_{\text {gap }}$ on $U$ is presented in the inset of (Fig. 6.1). The filling $n=0.9$ and Kondo coupling $J / W=0.2$ are fixed. While the general trend of $\Phi$ is a decrease with $U$, a peculiar feature arises between $U / W=0.1$ and $U / W=J / W=0.2$. There the anomalous expectation value displays a non-monotonous behavior and a local maximum around $U=J$. It further illustrates the unconventional nature of the superconducting state. This feature is likely to be related to local spin fluctuations, but a detailed explanation can unfortunately not be inferred from the standard expectation values of the system. In the previous chapter, local pairing in the simple KLM is identified to appear among heavy quasiparticles. Due to the mixed $c$ - $f$-character of these quasiparticles, a reduced on-site pair expectation value of the conduction electrons does thus not necessarily correlate with a reduced superconducting gap in the DOS, as long as $\Phi$ is finite. This is underpinned by the results for $\Delta_{\text {gap }}$ in the inset of (Fig. 6.1): While $\Phi$ decreases, $\Delta_{\text {gap }}$ does actually increase monotonically and saturates around $U \geq W$. This behavior can be understood as follows. An increasing local repulsion suppresses on-site charge fluctuations due to hopping and the local spin-density of conduction electrons becomes more of a local spin- $1 / 2$ character close to half filling. Thereby, local spin-fluctuations are enhanced and $c$ - $f$-singlet states favored - both are a necessary ingredient for pairing.

The dynamic gap function in (Fig. 6.1) offers more insight to the pairing. The lowfrequency range of $\operatorname{Re} \Delta(\omega)$ has been identified to be responsible for pairing among heavy quasiparticles. It changes sign at the effective quasiparticle bandwidth and quickly decays at higher energies for $U=0$. By increasing $U$ up to the bandwidth, $\operatorname{Re} \Delta(\omega)$ becomes primarily broadened. Together with $T_{0}$, the sign change shifts to higher energies. Even its value at $\omega=0$ attains slightly higher values for larger $U$, which is consistent with an increased gapwidth. For large $U / W=1.0$, an additional zero in the gapfunction emerges at high energies of order $U$.

The development of the superconducting state can additionally be traced by the evolution of corresponding spectral functions. The spectral functions on the complete energy scale 


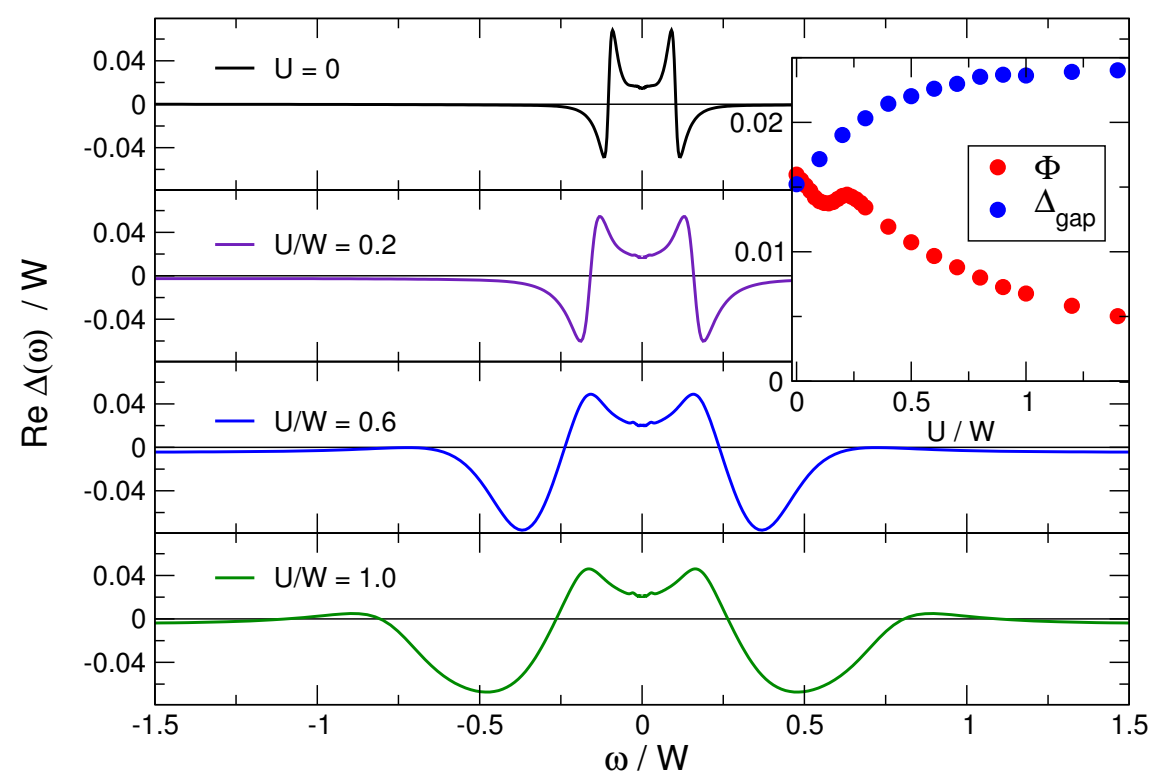

Figure 6.1: Real part of the dynamic gap function for several $U$ and fixed filling $n=0.9$ and Kondo coupling $J / W=0.2$. Inset: Development of $\Phi=\left\langle c_{\uparrow} c_{\downarrow}\right\rangle$ and the superconducting gap $\Delta_{\text {sc }}$ with increasing Coulomb repulsion $U$.

for $U / W=0.2,1.0$ are shown in (Fig. 6.2), while (Fig. 6.3) shows a closeup around the Fermi energy and the superconducting gap. On the overall scale, an increased $U$ broadens especially the upper band, but also leads to a more incoherent structure in the lower band, whereas the latter is relatively fixed at its position. The upper band, on the other hand, is shifted to higher energies due to the Coulomb repulsion and leaves some incoherent weight around $\omega \approx 0.20 W$ for large $U$, cf. the closeup for $U / W=1.0$. The most interesting development can be observed in the closeups around the Fermi energy. The flat and sharp quasiparticle bands at the gap edges are, for larger $U$, increasingly bended around $\varepsilon=1.0$. This behavior reflects a reduced effective mass compatible with the increase of $T_{0}$. Thereby the upper part of the flat band respectively the quasiparticle peak acquire more spectral weight, i.e., a larger fraction of heavy quasiparticles can take part in the superconducting condensate and the gap grows up to a saturation value.

In summary, the locally induced pairing of conduction electrons is - apart from a small local maximum - suppressed by a local Coulomb repulsion $U$, as expected. Nevertheless, the gap width increases with $U$, which may be interpreted in terms of $c$ - $f$-pairing and increased local spin fluctuations. 

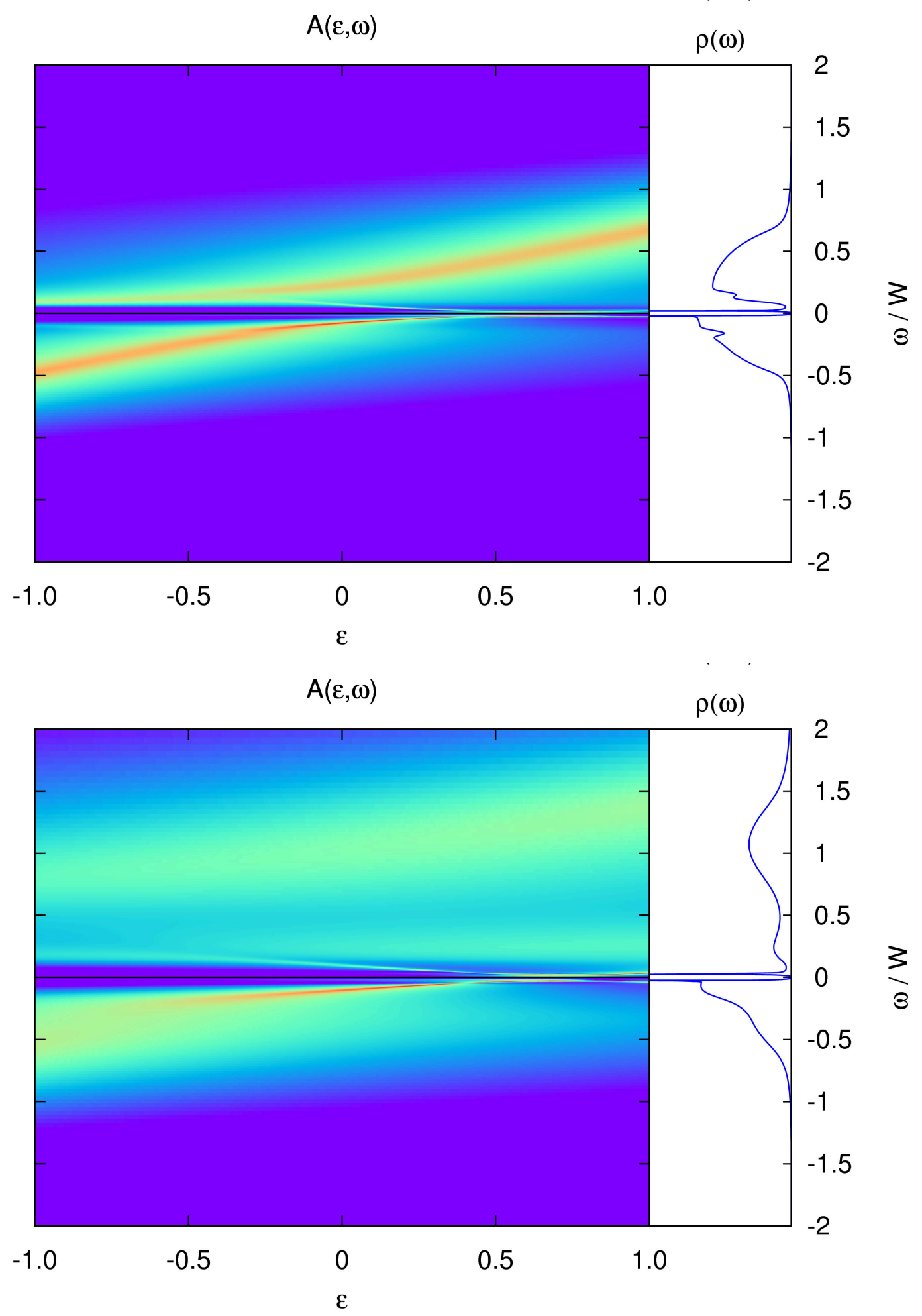

Figure 6.2: Spectral functions for the KLM $(n=0.9, J / W=0.2)$ in the SC phase with repulsive $U / W=0.2$ (top) and $U / W=1.0$ (bottom). The color scale is the same as in (Fig. 5.4). 

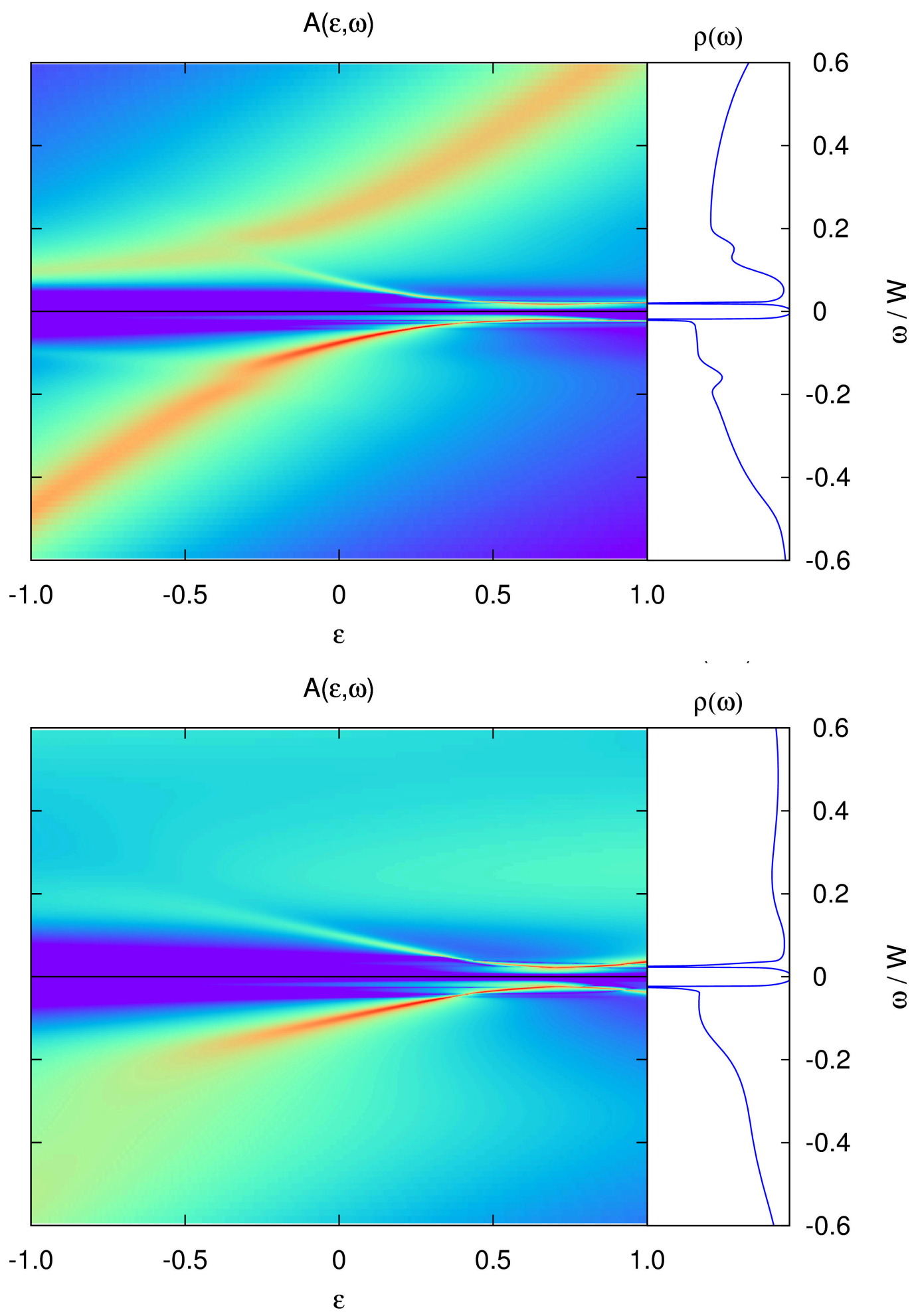

Figure 6.3: Closeups of (Fig. 6.2) on a smaller energy scale around the Fermi energy. The color scale is the same as in (Fig. 5.4). 


\subsection{Destabilized Pairing by Holstein Phonons}

The influence of an electron-phonon coupling by a Holstein term has been discussed for the paramagnetic phase in Sec. 3.2.2. The coupling to an Einstein mode does actually strongly suppress the coherence scale $T_{0}$ and associated spin fluctuations. This suppression is even stronger than exponential for large electron-phonon couplings. In the strong-coupling regime it is thereby even stronger than the enhancement by Coulomb repulsion. The influence of a local phonon mode with frequency $\omega_{0}$ and electron-phonon coupling $g$ on the superconducting state can thus be expected to be rather strong. The behavior of $\Phi$ and $\Delta_{\text {gap }}$ as function of $g$ is shown in the inset of (Fig. 6.4). The suppression of $T_{0}$ results in a decrease of pair correlations and in the reduction of the gap width. For larger electronphonon coupling it is again difficult to stabilize the solution against the formation of a charge-ordered state. It can thus not be clarified whether the pair correlations completely vanish at a critical $g_{c}$, or if it exhibits a local minimum and the unconventional pairing switches to a conventional phonon-mediated nature.

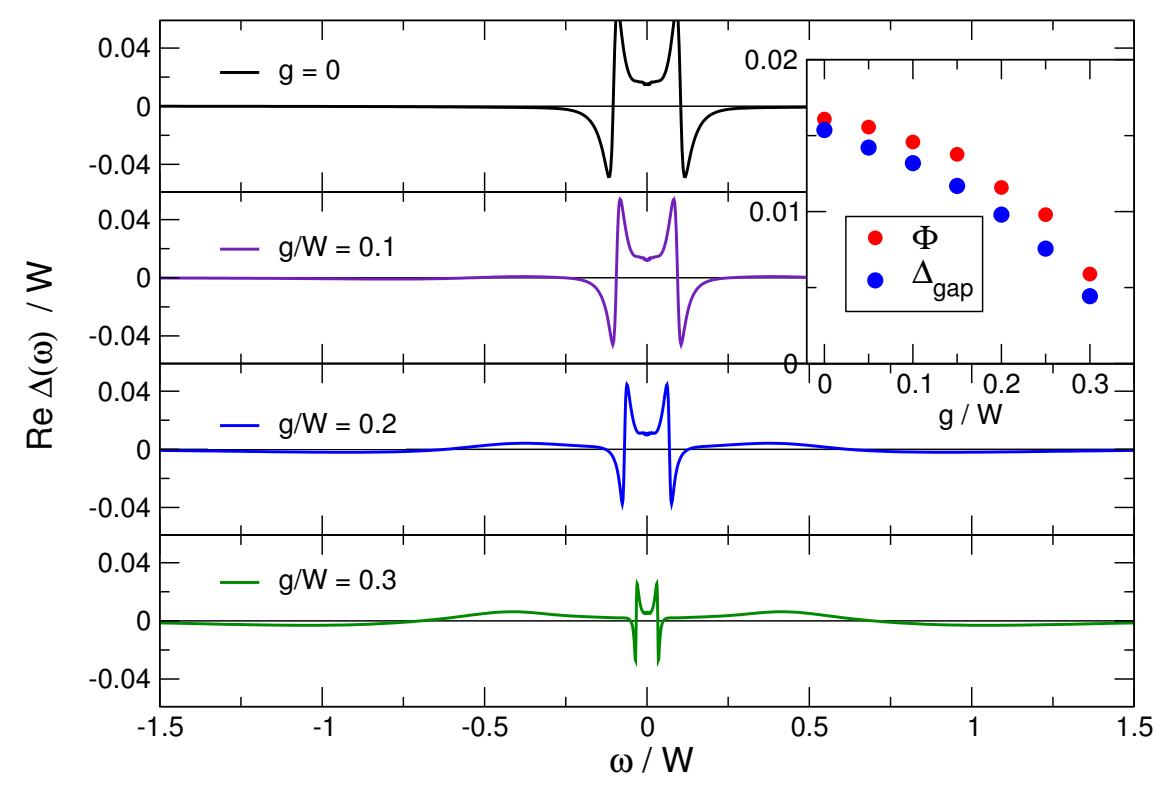

Figure 6.4: SC gap function for the KLM at $n=0.9, J / W=0.2$ with a Holstein term $\left(\omega_{0} / W=0.5\right)$ for different electron-phonon couplings $g / W=0,0.1,0.2,0.3$. The color scale is the same as in (Fig. 5.4). 
However, from results for an attractive $U$ it can - at least for the anti-adiabatic limit of the Holstein-Kondo lattice - be inferred, that another BEC-like superconducting solution emerges for larger $g$ : In the KLM with an attractive $U$, we find a superconducting solution with intact Kondo screening and a comparatively small order parameter for small $|U|<J$, while for large $|U| \gg J$ Kondo screening is almost completely suppressed and a BEC-like state with a large superconducting order parameter evolves. From this perspective, it would not be expected that superconductivity is completely suppressed but rather that its origin changes from spin fluctuations to phonons.

The presented results are somewhat surprising and do again illustrate the unconventional nature of the superconducting state in the KLM: An attractive interaction actually results in a suppression of pair correlations. Since the pairing stems from local spin fluctuations, it is actually not that unexpected on a second glance. The attractive interaction acting in the charge sector reduces spin fluctuations, which are a prerequisite for pairing in the KLM. Furthermore, the Eliashberg-type analysis also predicted that phonon-mediated pairing does not yield a solution to the gap equation for HF superconductors. The properties of Cooper pairing can be further characterized by the gap function shown for several electron-phonon couplings in (Fig. 6.4). Increasing $g$ has two effects: First, the zeros corresponding to the effective bandwidth shift to smaller energies for higher couplings. This observation is consistent with a reduced coherence scale $T_{0}$. Secondly, additional zeros in frequency space are induced. They probably reflect an effective interaction which changes sign more than once. Since a decay of $\operatorname{Re} \Delta$ is observed at even larger energies and not directly close to the zeros, this might be interpreted as a mixture of different attractive interactions which operate on different energy scales. However, the relevant low-energy scale on which the Kondo exchange interaction induces an effective attraction among the heavy quasiparticles is strongly reduced by enlarging $g$.

The spectral functions presented in (Fig. 6.5) further illustrate the influence of a Holsteinterm. For a small $g / W=0.1$, the structure almost equals the case of $g=0$, except for slightly narrowed structures. In the regime, where the electron phonon-coupling strongly suppresses the coherence scale, the effects become clearly visible. The hybridization gap is strongly quenched, and the side-resonances become increasingly sharp and compact on a reduced effective bandwidth. The superconducting gap is also reduced. At higher energies the spectrum is rather incoherent. In summary, it can be stated that the unconventional pairing mediated by local spin fluctuations is suppressed by a large coupling $g$ since the effective quasiparticle bandwidth, within which the pairing occurs, is increasingly reduced. 

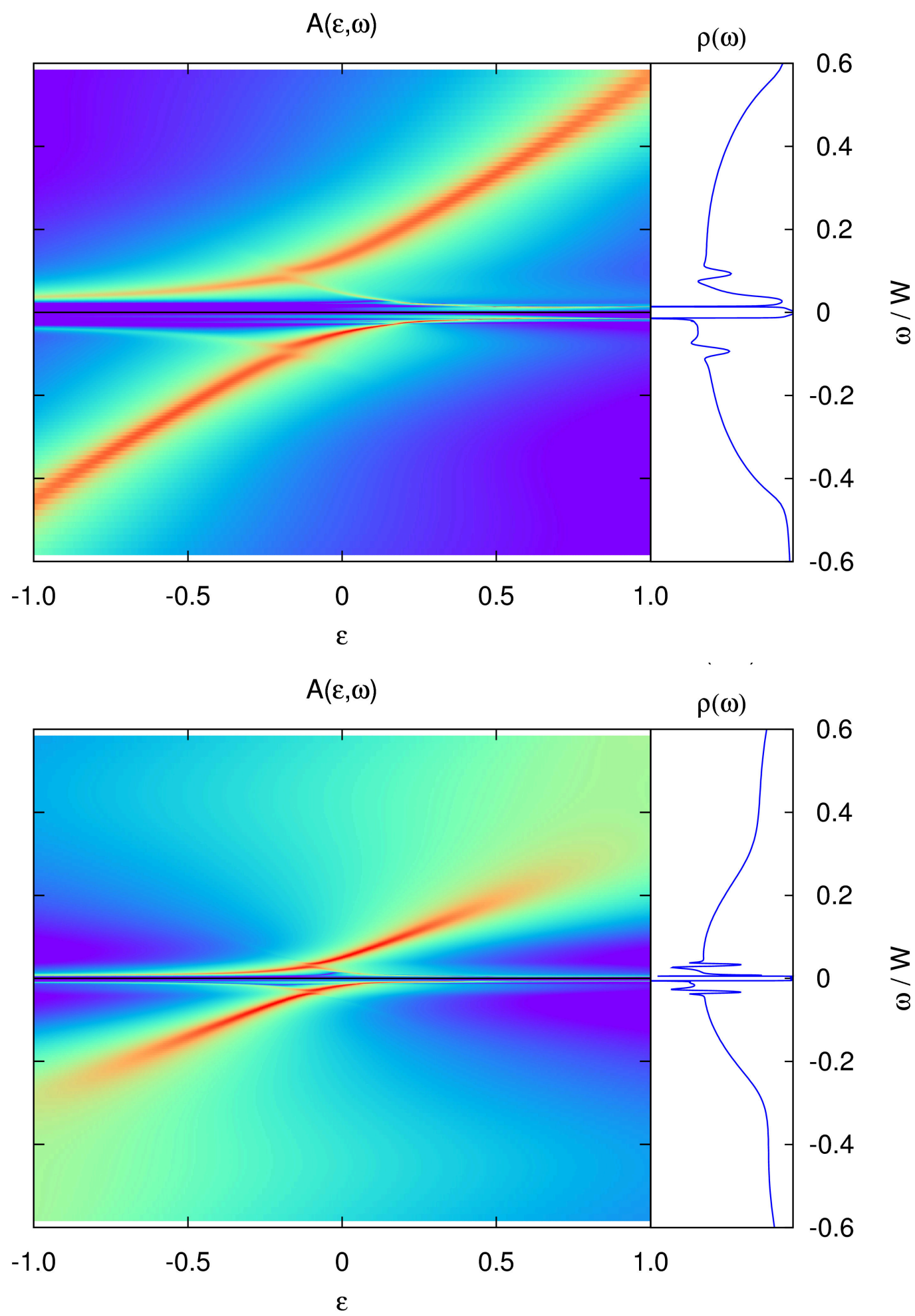

Figure 6.5: Spectral function $A(\varepsilon, \omega)$ and corresponding DOS $\rho(\omega)$ for the KLM with a Holstein term $\left(\omega_{0} / W=0.5\right)$ with $g / W=0.1$ (top) and $g / W=0.3$ (bottom). The filling $n=0.9$ and Kondo coupling $J / W=0.2$ are the same for both. Colors are scaled logarithmically as in (Fig. 5.4). 


\subsection{The underscreened Kondo lattice}

The KLM can be extended to local moments with a higher spin, e.g., $S=1$, which is applicable to some uranium-based [Per07] or neptunium-based [Tho12] HF compounds. The description is justified, if the $5 f$-electrons are well localized and most of the ions are in a $5 f^{2}$-configuration and form a $S=1$ state. In general, the $5 f$-electrons in actinide compounds can be localized, itinerant, or of a mixed nature in between, depending on the actual material. Although the underscreened KLM may not provide a general model for actinide-based HF compounds, it is nevertheless interesting to study the higher spin version of the KLM for two reasons. First, Kondo screening in a single-channel $S=1$ system is incomplete since only a spin- $1 / 2$ can be screened at each site. It is thus referred to as underscreened Kondo lattice. Two screening channels are necessary for a complete screening. A second interesting point is that it therefore allows for the coexistence of Kondo screening and magnetic ordering phenomena [Per07], whereas in the single-channel spin-1/2 KLM they typically compete.

Here the focus is on the superconducting phase of the underscreened KLM, which is indeed found to be stable. A comparison of several properties of the underscreened KLM and the simple KLM with $S=1 / 2$ is presented in (Fig. 6.6) for both the paramagnetic and superconducting phase. The parameters are $n=0.9$ and $J / W=0.3$. The figure parts (b), (d) and (f) in the right column show results for the paramagnetic phase. In part (b) the pseudogap in the paramagnetic DOS for $S=1$ is rather broad and reflects an enhanced energy scale $T_{0}$ of the heavy quasiparticles. Moreover, the DOS in the vicinity of the Fermi energy displays a steep step-like part, which is roughly follows the DOS for $S=1 / 2$. This "edge" is similar to the case of low filling factors in the spin-1/2 KLM, where underscreening effects may also play a role. In line with a larger coherence scale for a higher spin, the singularity in the self-energy moves to a higher frequency, cf. (d), (f). In the imaginary part (f) a little bit more structure is observable around the Fermi energy, especially a small hump close to the large peak. However, the spectral features in both cases are qualitatively rather similar, except for the larger coherence scale for $S=1$. The latter is expected from the known enhancement factor in $T_{0} \propto(N J)^{1 / N} \cdot \exp [-1 /(N J)]$ for a spin $S=N / 2$ Col13.

In the superconducting phase, cf. left column of (Fig. 6.6), the qualitative differences in the diagonal self-energy are neither very pronounced. The SC DOS displays a smaller gap in the high-spin case and the side-resonances vanish. A similar DOS is obtained for $S=1 / 2$ and a larger Kondo coupling. It can thus be attributed to an enhanced coherence scale $T_{0}$, too. Another fact underpinning this argument are the shifted zeros of both the off-diagonal self-energy (g) and the gap function (h). The latter observation is indicated by dashed lines and can be interpreted as an enlarged effective bandwidth, within which an attractive interaction is present among the heavy quasiparticles. The evolution of the anomalous expectation value $\Phi=\left\langle c_{\uparrow} c_{\downarrow}\right\rangle$ as function of $J$ is shown in part (i). It is generally 

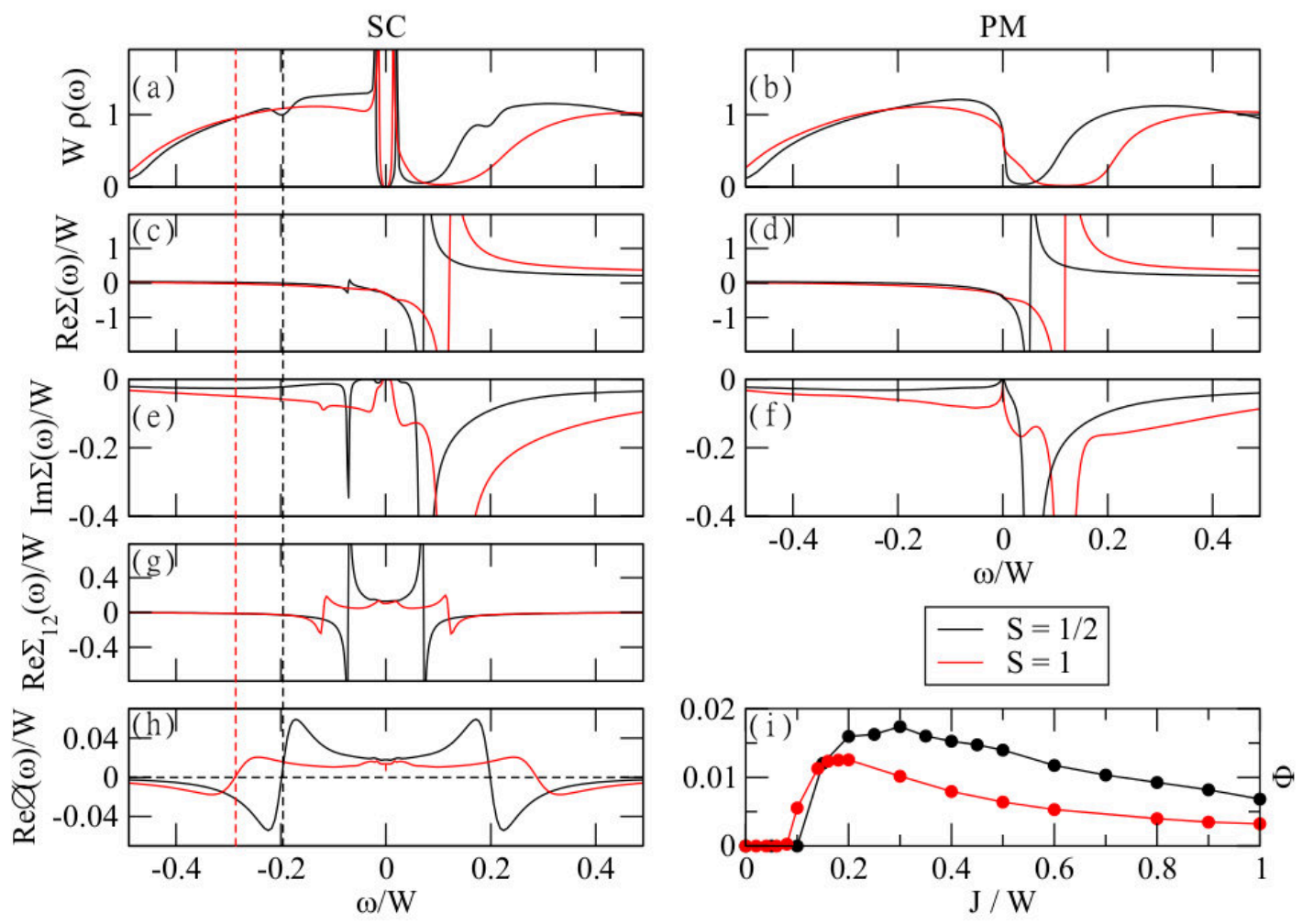

Figure 6.6: Comparison of properties of the KLM with $S=1$ (red) and $S=1 / 2$ (black) for fixed parameters $n=0.9$ and $J / W=0.3$ : SC DOS (a) and N DOS (b); corresponding real (c), (d) and imaginary parts (e), (f) of the diagonal self-energy; real part of the anomalous self-energy (g) and gap function (h) in the SC phase; (i) static anomalous expectation value $\Phi=\left\langle c_{\uparrow} c_{\downarrow}\right\rangle$ as function of $J$.

less pronounced for $S=1$ and sets in at a smaller $J$ compared to $S=1 / 2$. For large couplings, $\Phi$ decreases roughly proportional to $1 / J$.

Similar conclusions can be drawn from the spectral function in the superconducting phase of the underscreened KLM, cf. (Fig. 6.7). For a small coupling $J / W=0.1$ the band splitting and the corresponding side resonances are visible on a small energy scale around $\omega=0$, while the singularities at the gap edges are suppressed. The system is very sensitive to a variation of $J$ due to the enhancement factor of $T_{0}$ : Already an increase to $J / W=0.2$ completely broadens the spectrum and leaves a DOS with almost washed-out side resonances and no visible splitting of the bands. 

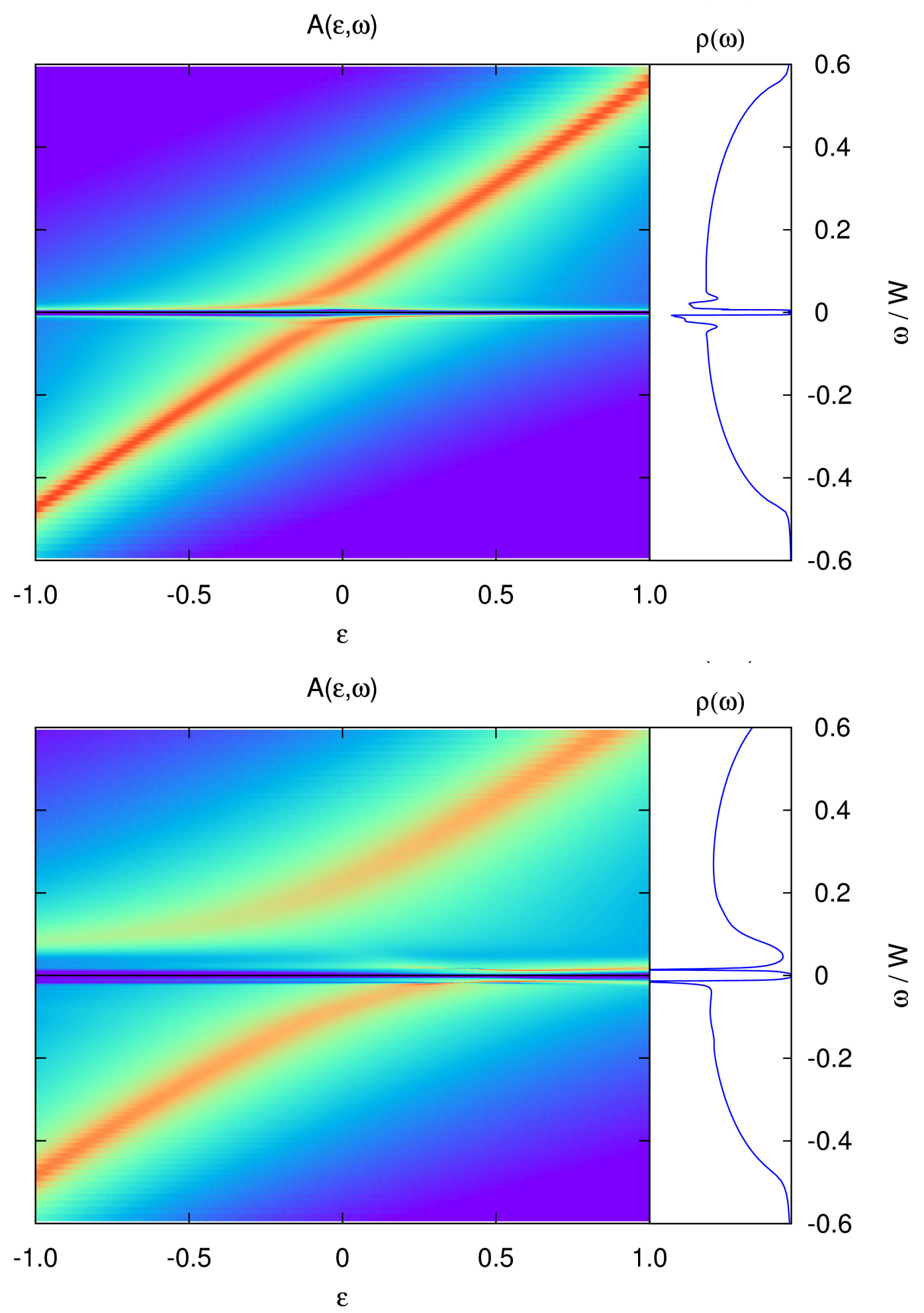

Figure 6.7: Spectral function $A(\varepsilon, \omega)$ and corresponding DOS $\rho(\omega)$ for the underscreened KLM with $J / W=0.1$ (top) and $J / W=0.2$ (bottom). The color scale is the same as in (Fig. 5.4). 



\section{Conclusion and Outlook}

In this thesis, the combination of DMFT and NRG was used to investigate superconductivity and antiferromagnetism in the Kondo-lattice model. The DMFT+NRG method is applied at the expense of loosing information about non-local correlations, while pairing in $\mathrm{CeCu}_{2} \mathrm{Si}_{2}$ and probably some other heavy-fermion superconductors seems to be mediated by non-local spin fluctuations [Sto11. Hence, it had not been clear before, if superconductivity can emerge from local correlations only. This question was affirmed in the present thesis.

The analysis of the paramagnetic state at zero temperature in chapter 3 reviewed the description of the electronic system as a Fermi liquid of heavy quasiparticles. Within DMFT + NRG a coherence scale $T_{0}$ can be identified for the heavy Fermi liquid. The known dependencies of $T_{0}$ on the filling $n$ of the conduction band and on the Kondo coupling $J$ were reproduced. Moreover, the influence of a local Coulomb repulsion in the conduction band and a coupling of conduction electrons to a bosonic Einstein mode were investigated. While the repulsion actually leads to an increase of $T_{0}$, thereby enhancing local spin fluctuations, a local boson-mediated attraction strongly suppresses $T_{0}$.

Besides these additional interactions, the heavy-fermion state itself is rather fragile against an intersite RKKY interaction, which also arises as a second-order process in the simple KLM without adding an explicit intersite term to the Hamiltonian. The DMFT+NRG method can simply be extended to handle antiferromagnetic Néel order. In the antiferromagnetically ordered phase at zero temperature and half filling, the Néel state was found to be stable for small couplings up to a critical $J_{c} / W \approx 0.275$. A peculiar feature arises in the density of states: We observe a "spin resonance" at energies of $\omega_{\mathrm{sr}} \sim \pm J / 2$. These resonances are the more pronounced, the higher the conduction band electrons are polarized - hence they are quite likely related to spin fluctuations. Close to half filling, the AF order persists in a metallic state for small Kondo coupling, or in an insulating sate for higher $J$. In the insulating state away from half filling, a resonance in the DOS was found in the majority-spin channel close to the Fermi energy. This insulating state is, however, very fragile and lifted by finite temperatures to a metallic state. In the latter case a transition from a local moment antiferromagnet to an itinerant heavy-fermion antiferromagnet is found within DMFT [Hos13], which is supported by the results obtained in this thesis. The itinerant antiferromagnetic phase is associated with incommensurate AF order, that is, the AF quantum critical point in the KLM is of spin-density-wave type. The strongly enhanced spin fluctuations close to the critical point motivate the search for spin-fluctuation mediated 
superconductivity.

As a main result of the present thesis, such a superconducting state at zero temperature was found to be stable in a large parameter regime within the DMFT+NRG approach. The pair correlations are indeed the most pronounced in the region of the parameter space, where AF order breaks down. Although only even-frequency singlet $s$-wave superconductivity can be treated within the utilized approach, the superconducting state was actually found to be closely related to local spin fluctuations, which act as retarded "glue" for $s$-wave pairing of heavy quasiparticles. This is a novel mechanism for superconductivity in HF systems. Similar to the antiferromagnetic case, resonances were observed in the density of states at energies related to the spin-fluctuation spectrum. These resonances were interpreted by means of a static mean-field description as a separation of the flat quasiparticle bands from the strongly dispersive part of the bandstructure. Such a structure is also observed in the spectral functions obtained by DMFT. Therefore, we drew the conclusion that the pairing appears among the heavy quasiparticles. This conclusion is underpinned by sign changes of the dynamic gap function at the very frequencies, where the resonances respectively the band splitting is observed. In addition, the observed rule that the critical temperature $T_{c}$ for the superconducting state is generally lower than the coherence scale $T_{0}$, indicated that the heavy-fermion state is a prerequisite for pairing in the Kondo-lattice model. Moreover, the ratio $\Delta_{\text {gap }} / T_{c}$ of the gapwidth and critical temperature is strongly enhanced over the universal BCS ratio. This fact is associated with a non-phononic origin of the attractive interaction. Surprisingly, the range of both $\Delta_{\text {gap }} / T_{c}$ and $T_{c}$ itself, which is of the order of a few Kelvin, roughly agree with experimental results. In summary, superconducting state was identified to be highly unconventional.

Further evidence for a local spin-fluctuation-mediated pairing was provided by two complementary viewpoints. First, an illustrative retarded pairing mechanism via virtual triplet excitations was proposed from strong-coupling arguments. Secondly, an Eliashberg-type treatment in combination with local Fermi-liquid approximations yielded a non-trivial solution of the gap equation, i.e., $T_{c}>0$, provided that a HF liquid is present and that the spin fluctuations decay rapidly on a small energy scale.

We further found that the influence of additional interactions on the superconducting state does not follow conventional behavior. The effects of these interactions on quasiparticle pairing are effectively characterized by their influence on the coherence scale $T_{0}$. A local Coulomb repulsion admittedly suppresses the local pairing in the conduction band but enhances the superconducting gap in the density of states. If superconductivity is interpreted in terms of $c$ - $f$-pairing, this might be understood as an increasing $f$-character of the Cooper pairs. In fact, spin fluctuations and thereby the pairing mechanism are supported by a local repulsion. Another important interaction is the electron-phonon coupling by a Holstein term. Its effect on the unconventional superconducting state is rather unexpected: An increasing electron-phonon coupling strongly suppresses the spinfluctuations and thereby the pairing mechanism instead of enhancing the pair correlations. 
In spectral functions of the KLM this behavior becomes apparent by a reduced effective bandwidth of the heavy quasiparticles. The unusual effects of both additional interactions underline the unconventional nature of $s$-wave superconductivity in the KLM.

The pairing mechanism still holds for a higher spin $S=1$ in the KLM. There we found essentially a qualitative agreement of the superconducting state with the $S=1 / 2$ case, apart from enhancement factors with respect to the coherence scale. As the underscreened Kondo-lattice features the coexistence of the Kondo-screening and magnetic order [Per07, and since the proposed pairing mechanism originates from the Kondo effect, the $S=1$ KLM constitutes a potential candidate for the coexistence of antiferromagnetism and superconductivity. However, this issue remains a challenge for future research.

The arising spin fluctuations close to the AF critical point enhance pair correlations among heavy quasiparticles, which require an intact Fermi-liquid state. This finding would underline a clear-cut criterion for superconductivity above QCPs in HF systems: Usually, HF superconductivity is not found above local QCPs, where the Kondo effect is critically destroyed Geg08. Within the Kondo-lattice model, superconductivity requires a Fermiliquid state and hence the Kondo effect to be intact. A "Kondo-breakdown" QCP would thus not feature unconventional superconductivity mediated by local spin fluctuations at the righthand side of the QCP. Both are necessary, spin fluctuations as "glue" and a underlying Fermi liquid of heavy quasiparticles. However, a single HF compound has been found recently, in which superconductivity seems to arise from fluctuations associated with a "Kondo-breakdown" QCP, namely CeRhIn 5 [Par11]. The scenario proposed in the present work, does certainly not apply for superconductivity close to local QCPs.

The presented results raise several questions, which remain to be addressed in the future. As the Kondo-lattice model describes the effective low-energy physics of the periodic Anderson model in the Kondo limit, a superconducting state should also be obtained in this very limit of the PAM. In the PAM it would be possible to study the interplay of pair correlations with charge fluctuations, which play an increasingly important role away from the Kondo limit of the PAM. Another line of research is to take into account non-local correlations. For this purpose, one needs to extend the DMFT method to clusters and utilize quantum cluster solvers, like quantum Monte Carlo methods. Another possibility is to resort to different methods beyond DMFT, like the dual-fermion method [Rub08] or the variational cluster approximation [S0́5]. With these methods it can be examined, whether superconductivity in models for heavy-fermion systems survives under the influence of non-local correlations. These methods further allow for an anisotropic, e.g., $d$-wave gap function.

Nevertheless, also an anisotropic pairing may originate from local spin fluctuations. Anisotropy itself may, e.g., be induced by other effects, which are not necessarily the true origin of a superconducting "glue". Even a Kondo interaction which is anisotropic due to the crystal structure, could yield an anisotropic gap function. The nodes of the gap 
function in frequency space, which we observed, may also translate into experimentally observed features of HF superconductors such as nodes of the gap function on the Fermi surface. These alternative scenarios of anisotropic pairing - albeit with a local origin - in $\mathrm{HF}$ superconductors are probably also worth investigating in parallel to the main route of current research.

Along these lines, this thesis shall end with the words of William James and Douglas Adams:

"Truth, as any dictionary will tell you, is a property of certain of our ideas. It means their 'agreement', as falsity means their disagreement, with 'reality.' " - William James

"Reality is frequently inaccurate." - Douglas Adams, in The Restaurant at the End of the Universe 
Appendices 



\section{A Details of the DMFT Setup}

In DMFT the local lattice Green's function $G_{\text {loc }}(\omega)$ needs to be calculated. This can be done by summation over the momentum,

$$
G_{\mathrm{loc}}(z)=\sum_{\mathbf{k}} G_{\mathbf{k}}(z)=\sum_{\mathbf{k}}\left(\boldsymbol{\zeta}-\varepsilon_{\mathbf{k}} \boldsymbol{\tau}_{3}\right)^{-1},
$$

with $\boldsymbol{\zeta}=z \mathbf{1}+\mu \boldsymbol{\tau}_{3}-\boldsymbol{\Sigma}(z)$, the chemical potential $\mu$, and the Pauli-matrix $\boldsymbol{\tau}_{3}$ and self-energy $\boldsymbol{\Sigma}$ as defined in Sec. 2.3. The summation can be carried out as integral over energies,

$$
G_{\text {loc }}(z)=\int_{-\infty}^{\infty} \mathrm{d} \varepsilon \rho_{0}(\varepsilon)\left(\hat{\zeta}-\varepsilon_{\mathbf{k}} \tau_{3}\right)^{-1}
$$

with the density of states $\rho_{0}(\varepsilon)$. In the paramagnetic case, the $\varepsilon$-summation results in

$$
G_{\mathrm{loc}}(z)=\int_{-\infty}^{\infty} \mathrm{d} \varepsilon \frac{\rho_{0}(\varepsilon)}{\zeta-\varepsilon}=\mathcal{H}\left[\rho_{0}\right](\zeta)
$$

where the Hilbert transform $\mathcal{H}$ for a general function $f(z)$ is defined as

$$
\mathcal{H}[f](z)=\int_{-\infty}^{\infty} \mathrm{d} \varepsilon \frac{f(\varepsilon)}{z-\varepsilon} .
$$

In some cases the Hilbert transform of the DOS is known analytically, so that this form of the integrand is advantegous. However, for the matrix structure in the superconducting case, such a form is not retained due to the matrix inversion. One can nevertheless perform a partial fraction decomposition so as to obtain the Green's function matrix elements as Bau11

$$
\begin{aligned}
G_{11}(\omega) & =A_{G} \mathcal{H}\left[\rho_{0}\right]\left(\varepsilon_{+}\right)+B_{G} \mathcal{H}\left[\rho_{0}\right]\left(\varepsilon_{-}\right) \quad \text { and } \\
G_{21}(\omega) & =A_{F} \mathcal{H}\left[\rho_{0}\right]\left(\varepsilon_{+}\right)+B_{F} \mathcal{H}\left[\rho_{0}\right]\left(\varepsilon_{-}\right)
\end{aligned}
$$


with
$A_{G}=\frac{\zeta_{2}+\varepsilon_{+}}{\varepsilon_{+}-\varepsilon_{-}}$
$B_{G}=\frac{\zeta_{2}+\varepsilon_{-}}{\varepsilon_{-}-\varepsilon_{+}}$
$A_{F}=\frac{\Sigma_{21}}{\varepsilon_{+}-\varepsilon_{-}}$
$B_{F}=\frac{\Sigma_{21}}{\varepsilon_{-}-\varepsilon_{+}}$

and

$$
\varepsilon_{ \pm}=\frac{\zeta_{1}-\zeta_{2}}{2} \pm \frac{1}{2} \sqrt{\left(\zeta_{1}+\zeta_{2}\right)^{2}-4 \Sigma_{12} \Sigma_{21}}
$$




\section{B NRG Setup for Superconducting Leads}

In the logarithmic discretization and in the derivation of the Wilson-chain coefficients we closely follow the scheme described in [Bul08, Bul97]. As an extension, also off-diagonal components are included here. We thereby allow for superconductivity in addition to a frequency-dependent DOS, which is needed for an application of the NRG as an impurity solver in DMFT.

\section{B.1 Logarithmic discretization}

Considering the band and hybirization part of a single impurity Anderson model in a superconducting medium, a possible generalised form is given by

$$
\begin{aligned}
H_{\text {band }}+H_{\text {hyb }}= & \sum_{\sigma} \int \mathrm{d} \omega \omega a_{\omega \sigma}^{\dagger} a_{\omega \sigma}+\int \mathrm{d} \omega \sqrt{\Gamma(\omega) / \pi}\left(d_{\sigma}^{\dagger} a_{\omega \sigma}+a_{\omega \sigma}^{\dagger} d_{\sigma}\right) \\
& -\int \mathrm{d} \omega \Delta(\omega)\left(a_{\omega \uparrow}^{\dagger} a_{\omega \downarrow}^{\dagger}+a_{\omega \downarrow} a_{\omega \uparrow}\right),
\end{aligned}
$$

where $\omega$ denotes the physical energy, which lies within the interval $[-1,1]$ in terms of half the bandwith $D$. The hybridization function is defined as $\Gamma(\omega)=\pi \rho_{0}(\omega)[V(\omega)]^{2}=$ $\pi \sum_{\mathbf{k}} V_{\mathbf{k}}^{2} \delta\left(\omega-\varepsilon_{\mathbf{k}}\right)$ and $\Delta(\omega)$ is the energy dependent paring amplitude. The pairing phase is assumed to be constant in the whole frequency range.

A change of variables from $\omega$ to $\varepsilon$ yields a Hamiltonian

$$
\begin{aligned}
H_{\text {band }}+H_{h y b}= & \sum_{\sigma} \int \mathrm{d} \varepsilon g(\varepsilon) a_{\varepsilon \sigma}^{\dagger} a_{\varepsilon \sigma}+\int \mathrm{d} \varepsilon h(\varepsilon)\left(d_{\sigma}^{\dagger} a_{\varepsilon \sigma}+a_{\varepsilon \sigma}^{\dagger} d_{\sigma}\right) \\
& -\int \mathrm{d} \varepsilon \tilde{\Delta}(\varepsilon)\left(a_{\varepsilon \uparrow}^{\dagger} a_{\varepsilon \downarrow}^{\dagger}+a_{\varepsilon \downarrow} a_{\varepsilon \uparrow}\right)
\end{aligned}
$$


which is equivalent to $(\mathrm{B} .2)$ if the three following conditions are fulfilled,

$$
\begin{aligned}
& \Gamma(\omega)=\pi \frac{\mathrm{d} \varepsilon(\omega)}{\mathrm{d} \omega} h[\varepsilon(\omega)]^{2} \\
& \varepsilon(\omega)=g^{-1}(\omega) \\
& \tilde{\Delta}[\varepsilon(\omega)]=\Delta(\omega),
\end{aligned}
$$

which has been shown by [Bul97]. Both $\omega$ and the fictious energy $\varepsilon$ run from -1 to 1 and are equal at each discretization point $x_{n}$. When $h(\varepsilon)$ is required to be a stepwise function with a constant value in each discretization interval, the impurity will only couple to the average state within each discretization interval. The average values of $h(\varepsilon)$ are

$$
h_{n}^{ \pm 2}=\frac{1}{d_{n}} \int^{ \pm, n} \mathrm{~d} \omega \frac{\Gamma(\omega)}{\pi} .
$$

$d_{n}$ is the width of the discretization interval and, $\pm n$ denotes the boundaries of the positive and negative discretization interval, respectively. The average states are obtained after discretizing the diagonal part of $H_{\text {band }}$ :

$$
\int_{-1}^{1} \mathrm{~d} \varepsilon g(\varepsilon) c_{\varepsilon}^{\dagger} c_{\varepsilon} \longrightarrow \sum_{n}\left(\xi_{n}^{+} a_{n}^{\dagger} a_{n}+\xi_{n}^{-} b_{n}^{\dagger} b_{n}\right), \quad \xi_{n}^{ \pm}=\frac{1}{d_{n}} \int^{ \pm, n} \mathrm{~d} \varepsilon g(\varepsilon)
$$

In each interval $\varepsilon, \omega \in\left[x_{n+1}, x_{n}\right]$ the relation

$$
\Gamma(\omega)=\pi \frac{\mathrm{d} \varepsilon}{\mathrm{d} \omega} h_{n}^{ \pm 2} \leftrightarrow \mathrm{d} \varepsilon=\frac{\Gamma[g(\varepsilon)]}{\pi h_{n}^{ \pm 2}} \mathrm{~d} g
$$

applies. Thus,

$$
\xi_{n}^{ \pm}=\frac{1}{d_{n}} \int^{ \pm, n} \mathrm{~d} g g \frac{\Gamma(g)}{\pi h_{n}^{ \pm 2}} .
$$

The integration boundaries remain the same after the change of the integration variable as $g\left( \pm x_{n}\right)= \pm x_{n}$. Renaming $g$ with $\omega$, the coefficients of the discretized Hamiltonian are finally obtained as

$$
\xi_{n}^{ \pm}=\frac{\int^{ \pm, n} \omega \Gamma(\omega) \mathrm{d} \omega}{\int^{ \pm, n} \Gamma(\omega) \mathrm{d} \omega} .
$$

An analogous procedure is possible for the off-diagonal pairing term of the band Hamilto- 
nian,

$$
\begin{aligned}
& \int_{-1}^{1} \mathrm{~d} \varepsilon \tilde{\Delta}(\varepsilon)\left(c_{\varepsilon \uparrow}^{\dagger} c_{\varepsilon \downarrow}^{\dagger}+c_{\varepsilon \downarrow} c_{\varepsilon \uparrow}\right) \longrightarrow \sum_{n} \Delta_{n}^{+}\left(a_{n \uparrow}^{\dagger} a_{n \downarrow}^{\dagger}+\text { h.c. }\right)+\Delta_{n}^{-}\left(a_{n \uparrow}^{\dagger} a_{n \downarrow}^{\dagger}+\text { h.c. }\right) \\
& \Delta_{n}^{ \pm}=\frac{1}{d_{n}} \int^{ \pm, n} \mathrm{~d} \varepsilon \tilde{\Delta}(\varepsilon) .
\end{aligned}
$$

The change of the integration variable

$$
\Delta_{n}^{ \pm}=\frac{1}{d_{n}} \int^{ \pm, n} \tilde{\Delta}[\varepsilon(\omega)] \frac{\mathrm{d} \varepsilon}{\mathrm{d} \omega} \mathrm{d} \omega=\frac{1}{d_{n}} \int^{ \pm, n} \Delta(\omega) \frac{\Gamma(\omega)}{\pi h_{n}^{ \pm 2}} \mathrm{~d} \omega
$$

results in the expression

$$
\Delta_{n}^{ \pm}=\frac{\int^{ \pm, n} \Gamma(\omega) \Delta(\omega) \mathrm{d} \omega}{\int^{ \pm, n} \Gamma(\omega) \mathrm{d} \omega}
$$

which is equivalent to the one for the kinetic part of the Hamiltonian, replacing energy $\omega$ with pairing $\Delta(\omega)$. Altogether the discretized band Hamiltonian reads

$$
\begin{aligned}
H_{\mathrm{band}} & =\sum_{n}\left(\xi_{n}^{+} a_{n}^{\dagger} a_{n}+\xi_{n}^{-} b_{n}^{\dagger} b_{n}\right) \\
& +\sum_{n} \Delta_{n}^{+}\left(a_{n \uparrow}^{\dagger} a_{n \downarrow}^{\dagger}+\text { h.c. }\right)+\Delta_{n}^{-}\left(a_{n \uparrow}^{\dagger} a_{n \downarrow}^{\dagger}+\text { h.c. }\right) .
\end{aligned}
$$

Finally, the hybridization term has to be discretized:

$$
\int_{-1}^{1} \mathrm{~d} \varepsilon h(\varepsilon) d_{\sigma}^{\dagger} c_{\varepsilon \sigma} \longrightarrow \frac{1}{\sqrt{\pi}} d_{\sigma}^{\dagger} \sum_{n}\left(\gamma_{n}^{+} a_{n \sigma}+\gamma_{n}^{-} b_{n \sigma}\right), \quad \gamma_{n}^{ \pm}=\sqrt{\int^{ \pm, n} \mathrm{~d} \omega \Gamma(\omega)}
$$

In order to apply an RG transformation, the Hamiltonian has to be transformed from the 'star basis' into the Wilson chain basis where only the first state of the chain is directly coupled to the impurity.

\section{B.2 Mapping to the Wilson-chain}

In the folllowing I use Nambu-spinors

$$
\underline{f}=\left(\begin{array}{c}
f_{\uparrow} \\
f_{\downarrow}^{\dagger}
\end{array}\right) \quad \underline{f}^{\dagger}=\left(f_{\uparrow}^{\dagger}, f_{\downarrow}\right)
$$


which obey the anticommutator relation

$$
\begin{aligned}
& {\left[\underline{f}, \underline{f}^{\dagger}\right]_{+}=\underline{f} \otimes \underline{f}^{\dagger}+\underline{f}^{\dagger} \otimes \underline{f}=\left(\begin{array}{l}
{\left[f_{\uparrow}, f_{\uparrow}^{\dagger}\right]_{+}} \\
{\left[f_{\downarrow}^{\dagger}, f_{\uparrow}^{\dagger}\right]_{+}}
\end{array} \begin{array}{l}
{\left[f_{\uparrow}, f_{\downarrow}\right]_{+}} \\
{\left[f_{\downarrow}^{\dagger}, f_{\downarrow}\right]_{+}}
\end{array}\right)=\mathbf{1}} \\
& {\left[\underline{f}, \underline{f}^{\dagger} \boldsymbol{A}\right]_{+}=\boldsymbol{A}} \\
& {\left[\boldsymbol{B} \underline{f}, \underline{f}^{\dagger} \boldsymbol{A}\right]_{+}=\boldsymbol{B} \boldsymbol{A} .}
\end{aligned}
$$

Thereby, the conduction part of the Hamiltonian, Eq.(B.9), can be written in a compact form,

$$
H=\sum_{n} \underline{a}_{n}^{\dagger} \Gamma_{n}^{+} \underline{a}_{n}+\underline{b}_{n}^{\dagger} \boldsymbol{\Gamma}_{n}^{-} \underline{b}_{n}-\xi_{n}^{+}-\xi_{n}^{-},
$$

where $\boldsymbol{\Gamma}_{n}^{ \pm}=\left(\begin{array}{cc}\xi_{n}^{ \pm} & -\Delta_{n}^{ \pm} \\ -\Delta_{n}^{ \pm} & -\xi_{n}^{ \pm}\end{array}\right)$

The Hamiltonian $\tilde{H}=H+\sum_{n} \xi_{n}^{+}+\xi_{n}^{-}$now has to be transformed from the star basis into the Wilson-chain basis, that is,

$$
\tilde{H}=\sum_{n} \underline{a}_{n}^{\dagger} \boldsymbol{\Gamma}_{n}^{+} \underline{a}_{n}+\underline{b}_{n}^{\dagger} \boldsymbol{\Gamma}_{n}^{-} \underline{b}_{n} \stackrel{!}{=} \sum_{n} \underline{f}_{n}^{\dagger} \varepsilon_{n} \underline{f}_{n}+\underline{f}_{n}^{\dagger} \boldsymbol{t}_{n} \underline{f}_{n+1}+\underline{f}_{n+1}^{\dagger} \boldsymbol{t}_{n}^{T} \underline{f}_{n}
$$

has to be fulfilled. For this purpose orthogonal transformations $\boldsymbol{U}$ and $\boldsymbol{V}$ are defined via

$$
\begin{aligned}
\underline{f}_{n} & =\sum_{m} \boldsymbol{U}_{n m} \underline{a}_{m}+\boldsymbol{V}_{n m} \underline{b}_{m} & \underline{f}_{n}^{\dagger} & =\sum_{m} \underline{a}_{m}^{\dagger} \boldsymbol{U}_{n m}^{T}+\underline{b}_{m}^{\dagger} \boldsymbol{V}_{n m}^{T} \\
\underline{a}_{n} & =\sum_{m} \boldsymbol{U}_{m n}^{T} \underline{f}_{m} & \underline{a}_{n}^{\dagger} & =\sum_{m} \underline{f}_{m}^{\dagger} \boldsymbol{U}_{m n} \\
\underline{b}_{n} & =\sum_{m} \boldsymbol{V}_{m n}^{T} \underline{f}_{m} & \underline{b}_{n}^{\dagger} & =\sum_{m} \underline{f}_{m}^{\dagger} \boldsymbol{V}_{m n} .
\end{aligned}
$$

Defining the spinor $\underline{f}_{0}$ which corresponds to the first chain site as

$$
\underline{f}_{0}:=\frac{1}{\sqrt{\xi_{0}}} \sum_{n} \gamma_{n}^{+} \underline{a}_{n}+\gamma_{n}^{-} \underline{b}_{n},
$$

a comparision of the coupling terms in the star and chain basis yields

$$
\boldsymbol{U}_{0 m}=\frac{\gamma_{n}^{+}}{\sqrt{\xi_{0}}} \mathbf{1}_{0 m} \quad \boldsymbol{V}_{0 m}=\frac{\gamma_{n}^{-}}{\sqrt{\xi_{0}}} \mathbf{1}_{0 m}
$$

where $\xi_{0}=\sum_{n}\left(\gamma_{n}^{+}\right)^{2}+\left(\gamma_{n}^{-}\right)^{2}=\int_{-1}^{1} \mathrm{~d} \varepsilon \Gamma(\varepsilon)$ is a normalization constant.

After the transformation of the annihilation operators in $\tilde{H}$ one has again to compare the 
terms in the star and chain basis. For $m=0$ this results in

$$
\sum_{n} \underline{a}_{n}^{\dagger} \boldsymbol{\Gamma}_{n}^{+} \boldsymbol{U}_{0 n}^{T}+\underline{b}_{n}^{\dagger} \boldsymbol{\Gamma}_{n}^{+} \boldsymbol{V}_{0 n}^{T}=\underline{f}_{0}^{\dagger} \varepsilon_{0}+\underline{f}_{1}^{\dagger} \boldsymbol{t}_{0}^{T}
$$

and for $m>0$ it gives

$$
\sum_{n} \underline{a}_{n}^{\dagger} \boldsymbol{\Gamma}_{n}^{+} \boldsymbol{U}_{m n}^{T}+\underline{b}_{n}^{\dagger} \boldsymbol{\Gamma}_{n}^{-} \boldsymbol{V}_{m n}^{T}=\underline{f}_{m}^{\dagger} \boldsymbol{\varepsilon}_{m}+\underline{f}_{m+1}^{\dagger} \boldsymbol{t}_{m}^{T}+\underline{f}_{m-1}^{\dagger} \boldsymbol{t}_{m-1} .
$$

By taking the anticommutator of $\underline{f}_{0}$ and Eq. (B.19),

$$
\boldsymbol{\varepsilon}_{0}=\sum_{n} \boldsymbol{U}_{0 n} \boldsymbol{\Gamma}_{n}^{+} \boldsymbol{U}_{0 n}^{T}+\boldsymbol{V}_{0 n} \boldsymbol{\Gamma}_{n}^{-} \boldsymbol{V}_{0 n}^{T}
$$

is determined. But as $\boldsymbol{U}_{0 n}$ is diagonal,

$$
\boldsymbol{\varepsilon}_{0}=\frac{1}{\xi_{0}} \sum_{n}\left[\left(\gamma_{n}^{+}\right)^{2} \boldsymbol{\Gamma}_{n}^{+}+\left(\gamma_{n}^{-}\right)^{2} \boldsymbol{\Gamma}_{n}^{-}\right]
$$

Furthermore, from Eq. B.19) follows

$$
\underline{f}_{1}^{\dagger} \boldsymbol{t}_{0}^{T}=\sum_{n} \underline{a}_{n}^{\dagger}\left(\boldsymbol{\Gamma}_{n}^{+} \boldsymbol{U}_{0 n}^{T} \varepsilon_{0}\right)+\underline{b}_{n}^{\dagger}\left(\boldsymbol{\Gamma}_{n}^{-} \boldsymbol{V}_{0 n}^{T} \varepsilon_{0}\right)
$$

which allows for determining the matrix elements

$$
\begin{aligned}
& \boldsymbol{U}_{1 n}^{T}=\left(\boldsymbol{\Gamma}_{n}^{+} \boldsymbol{U}_{0 n}^{T}-\boldsymbol{U}_{0 n}^{T} \varepsilon_{0}\right) \boldsymbol{t}_{0}^{-1} \\
& \boldsymbol{V}_{1 n}^{T}=\left(\boldsymbol{\Gamma}_{n}^{-} \boldsymbol{V}_{0 n}^{T}-\boldsymbol{V}_{0 n}^{T} \varepsilon_{0}\right) \boldsymbol{t}_{0}^{-1} .
\end{aligned}
$$

Taking the anticommutator of Eq. B.23) with the corresponding adjoint operators on both sides yields the relation

$$
\boldsymbol{t}_{0}^{T} \boldsymbol{t}_{0}=\sum_{n} \frac{\left(\gamma_{n}^{+}\right)^{2}}{\xi_{0}}\left[\left(\boldsymbol{\Gamma}_{n}^{+}\right)^{T}-\boldsymbol{\varepsilon}_{0}^{T}\right]\left[\boldsymbol{\Gamma}_{n}^{+}-\boldsymbol{\varepsilon}_{0}\right]+\frac{\left(\gamma_{n}^{-}\right)^{2}}{\xi_{0}}\left[\left(\boldsymbol{\Gamma}_{n}^{-}\right)^{T}-\boldsymbol{\varepsilon}_{0}^{T}\right]\left[\boldsymbol{\Gamma}_{n}^{-}-\boldsymbol{\varepsilon}_{0}\right]
$$

The chain hopping $\boldsymbol{t}_{0}$ is assumed to be diagonal, and can now be calculated by the equation above.

The anticommutator of $\underline{f}_{m}$ and Eq. B.20 enables one to determine $\varepsilon_{m}$,

$$
\boldsymbol{\varepsilon}_{m}=\sum_{n} \boldsymbol{U}_{m n} \boldsymbol{\Gamma}_{n}^{+} \boldsymbol{U}_{m n}^{T}+\boldsymbol{V}_{m n} \boldsymbol{\Gamma}_{n}^{-} \boldsymbol{V}_{m n}^{T}
$$


Moreover, from Eq. B.20 it follows that

$$
\begin{aligned}
& \boldsymbol{t}_{m} \boldsymbol{U}_{m+1, n}=\boldsymbol{U}_{m n}\left(\boldsymbol{\Gamma}_{n}^{+}\right)^{T}-\boldsymbol{\varepsilon}_{m}^{T} \boldsymbol{U}_{m n}-\boldsymbol{t}_{m-1}^{T} \boldsymbol{U}_{m-1, n} \\
& \boldsymbol{t}_{m} \boldsymbol{V}_{m+1, n}=\boldsymbol{V}_{m n}\left(\boldsymbol{\Gamma}_{n}^{-}\right)^{T}-\boldsymbol{\varepsilon}_{m}^{T} \boldsymbol{V}_{m n}-\boldsymbol{t}_{m-1}^{T} \boldsymbol{V}_{m-1, n} .
\end{aligned}
$$

According to the steps shown above one can derive a relation for the chain hopping

$$
\boldsymbol{t}_{m} \boldsymbol{t}_{m}^{T}=\sum_{n}\left(\boldsymbol{M}_{m n}^{T} \boldsymbol{M}_{m n}+\boldsymbol{N}_{m n}^{T} \boldsymbol{N}_{m n}\right)
$$

with

$$
\begin{aligned}
\boldsymbol{M}_{m n} & =\boldsymbol{\Gamma}_{n}^{+} \boldsymbol{U}_{m n}^{T}-\boldsymbol{U}_{m n}^{T} \boldsymbol{\varepsilon}_{m}-\boldsymbol{U}_{m-1, n}^{T} \boldsymbol{t}_{m-1} \\
\boldsymbol{N}_{m n} & =\boldsymbol{\Gamma}_{n}^{-} \boldsymbol{V}_{m n}^{T}-\boldsymbol{V}_{m n}^{T} \boldsymbol{\varepsilon}_{m}-\boldsymbol{V}_{m-1, n}^{T} \boldsymbol{t}_{m-1} .
\end{aligned}
$$

Altogether, a set of recursion relations for the orthogonal transformations $\boldsymbol{U}$ and $\boldsymbol{V}$ has been derived. Thereby it is possible to determine the operators and coefficients of the Wilson-chain in Eq. B.15). 


\section{Bibliography}

[All82] Allen, J. and Martin, Richard: Kondo Volume Collapse and the $\gamma-\alpha$ Transition in Cerium. Physical Review Letters (1982), vol. 49(15):pp. 1106-1110, URL http://link.aps.org/doi/10.1103/PhysRevLett.49.1106

[And61] Anderson, P.: Localized Magnetic States in Metals. Physical Review (1961), vol. 124(1):pp. 41-53, URL http://link.aps.org/doi/10.1103/PhysRev.124.41

[And75] Andres, K.; Graebner, J. and Otт, H.: 4f-Virtual-Bound-State Formation in CeAl_\{3\} at Low Temperatures. Physical Review Letters (1975), vol. 35(26):pp. 1779-1782, URL http://link.aps.org/doi/10.1103/PhysRevLett.35.1779

[And05] Anders, Frithjof and Schiller, Avraham: Real-Time Dynamics in QuantumImpurity Systems: A Time-Dependent Numerical Renormalization-Group Approach. Physical Review Letters (2005), vol. 95(19):p. 196801, URL http: //link.aps.org/doi/10.1103/PhysRevLett.95.196801

[And06] ANDERs, Frithjof and SchILleR, Avraham: Spin precession and real-time dynamics in the Kondo model:Time-dependent numerical renormalizationgroup study. Physical Review B (2006), vol. 74(24):p. 245113, URL http: //link.aps.org/doi/10.1103/PhysRevB.74.245113

[Ass99] AssaAd, F.: Quantum Monte Carlo Simulations of the Half-Filled TwoDimensional Kondo Lattice Model. Physical Review Letters (1999), vol. 83(4):pp. 796-799, URL http://prl.aps.org/abstract/PRL/v83/i4/p796_1

[Bar57a] BArdeEn, J.; CoOper, L. and Schrieffer, J.: Microscopic Theory of Superconductivity. Physical Review (1957), vol. 106(1):pp. 162-164, URL http://link.aps.org/doi/10.1103/PhysRev.106.162

[Bar57b] Bardeen, J.; Cooper, L. N. and Schrieffer, J. R.: Theory of Superconductivity. Physical Review (1957), vol. 108(5):pp. 1175-1204, URL http: //link.aps.org/doi/10.1103/PhysRev.108.1175

[Bau09] Bauer, J.; Hewson, A. and Dupuis, N.: Dynamical mean-field theory and numerical renormalization group study of superconductivity in the attractive Hubbard model. Physical Review B (2009), vol. 79(21), URL http://prb.aps. org/abstract/PRB/v79/i21/e214518

[Bau10a] BAUER, J.: Competing interactions and symmetry breaking in the HubbardHolstein model. EPL (Europhysics Letters) (2010), vol. 90(2):p. 27002, URL http://stacks.iop.org/0295-5075/90/i=2/a=27002

[Bau10b] BAUER, Johannes and Hewson, Alex C.: Competition between antiferromagnetic and charge order in the Hubbard-Holstein model. Physical Review B (2010), 
vol. 81(23), URL http://prb.aps.org/abstract/PRB/v81/i23/e235113

[Bau11] BAUER, Johannes; HAN, Jong and Gunnarsson, Olle: Quantitative reliability study of the Migdal-Eliashberg theory for strong electron-phonon coupling in superconductors. Physical Review B (2011), vol. 84(18), URL http://prb.aps org/abstract/PRB/v84/i18/e184531

[Bax08] BAxter, Rodney J.: Exactly Solved Models in Statistical Mechanics, Dover Publications (2008)

[Bed86] Bednorz, J. G. and Müller, K. A.: Possible high $\$ T \_c \$$ superconductivity in the Ba-La-Cu-O system. Zeitschrift für Physik B Condensed Matter (1986), vol. 64(2):pp. 189-193, URL http://link.springer.com/10.1007/ BF01303701

[Bla87] Blankenbecler, R.; Fulco, J.; Gill, W. and Scalapino, D.: Groundstate properties of the periodic Anderson model. Physical Review Letters (1987), vol. 58(4):pp. 411-414, URL http://link.aps.org/doi/10.1103/ PhysRevLett.58.411

[Bod10] Bodensiek, O; Pruschke, T and Žıtko, R: Superconductivity in the Kondo lattice model. Journal of Physics: Conference Series (2010), vol. 200(1):p. 012162, URL http://stacks .iop.org/1742-6596/200/i=1/a=012162

[Bod11] Bodensiek, Oliver; Žitko, Rok; Peters, Robert and Pruschke, Thomas: Lowenergy properties of the Kondo lattice model. Journal of Physics: Condensed Matter (2011), vol. 23(9):p. 094212, URL http://stacks.iop.org/0953-8984/ $23 / i=9 / a=094212$

[Bod13] Bodensiek, Oliver; Žitko, Rok; Vojta, Matthias; Jarrell, Mark and PrUschke, Thomas: Unconventional Superconductivity from Local Spin Fluctuations in the Kondo Lattice. Physical Review Letters (2013), vol. 110(14):p. 146406, URL http://link.aps.org/doi/10.1103/PhysRevLett.110.146406

[Bre78] Bredl, C. D.; Steglich, F. and Schotte, K. D.: Specific heat of concentrated kondo systems: (La, Ce)Al2 and CeAl2. Zeitschrift für Physik B Condensed Matterr Physik B Condensed Matter and Quanta (1978), vol. 29(4):pp. 327-340, URL http://link. springer .com/10.1007/BF01324030

[Bro90] Broholm, C.; Aeppli, G.; Kleiman, R.; Harshman, D.; Bishop, D.; Bucher, E.; Williams, D.; Ansaldo, E. and Heffner, R.: Anisotropic temperature dependence of the magnetic-field penetration in superconducting UPt_ $\{3\}$. Physical Review Letters (1990), vol. 65(16):pp. 2062-2065, URL http://link aps.org/doi/10.1103/PhysRevLett.65.2062

[Bul97] Bulla, R; Pruschke, Th and Hewson, A C: Anderson impurity in pseudo-gap Fermi systems. Journal of Physics: Condensed Matter (1997), vol. 9(47):pp. 10463-10474, URL http://stacks.iop.org/0953-8984/9/i=47/a=014

[Bul98] Bulla, R; Hewson, A C and Pruschke, Th: Numerical renormalization group calculations for the self-energy of the impurity Anderson model. Journal of Physics: Condensed Matter (1998), vol. 10(37):pp. 8365-8380, URL http: //stacks. iop.org/0953-8984/10/i=37/a=021

[Bul08] Bulla, Ralf; Costi, Theo and Pruschke, Thomas: Numerical renormaliza- 
tion group method for quantum impurity systems. Reviews of Modern Physics (2008), vol. 80(2):pp. 395-450, URL http://rmp.aps .org/abstract/RMP/v80/ i2/p395_1

[Bur00] Burdin, S.; Georges, A. and Grempel, D.: Coherence Scale of the Kondo Lattice. Physical Review Letters (2000), vol. 85(5):pp. 1048-1051, URL http://han.sub.uni-goettingen.de/han/52538_0/prl. aps.org/abstract/PRL/v85/i5/p1048_1

[Col13] Coleman, Piers: Introduction to Many Body Physics (2013), URL http://www physics.rutgers.edu/ coleman/620/mbody/pdf/bkx.pdf

[Don77] Doniach, S: The Kondo lattice and weak antiferromagnetism. Physica BC (1977), vol. 91:pp. 231-234, URL http://dx.doi.org/10.1016/0378-4363(77) 90190-5

[Don12] Dong, R; Otsuki, J and Savrasov, S Y: Scaling Between Periodic Anderson and Kondo Lattice Models (2012):pp. 1-9, URL http://arxiv.org/abs/1212.5867

[dV91] DE Visser, A. and Franse, J.J.M.: Uranium-based heavy-fermion superconductors: an experimental survey. Journal of Magnetism and Magnetic Materials (1991), vol. 100(1-3):pp. 204-217, URL http://dx.doi.org/10.1016/ 0304-8853(91) 90821-Q

[Eco90] Econommou, E.N.: Green's Functions in Quantum Physics, Springer-Verlag (1990)

[Eli69] EliashberG, G.M.: Nonstationary Equations for Superconductors with Low Concentration of Paramagnetic Impurities. Journal of Experimental and Theoretical Physics (1969), vol. 28(6):p. 1298

[Faz91] FazeKas, P. and Müller-Hartmann, E.: Magnetic and non-magnetic ground states of the Kondo lattice. Zeitschrift für Physik B Condensed Matterr Physik B Condensed Matter (1991), vol. 85(2):pp. 285-300, URL http://link. springer. com/10.1007/BF01313231

[Fli08] Flint, Rebecca; Dzero, M. and Coleman, P.: Heavy electrons and the symplectic symmetry of spin. Nature Physics (2008), vol. 4(8):pp. 643-648, URL http: //dx.doi.org/10.1038/nphys1024

[Fli10] Flint, Rebecca and Coleman, Piers: Tandem Pairing in Heavy-Fermion Superconductors. Physical Review Letters (2010), vol. 105(24):p. 246404, URL http://link.aps.org/doi/10.1103/PhysRevLett.105.246404

[Fre93] Freericks, J.; Jarrell, M. and Scalapino, D.: Holstein model in infinite dimensions. Physical Review B (1993), vol. 48(9):pp. 6302-6314, URL http: //link.aps.org/doi/10.1103/PhysRevB.48.6302

[Fri52] FRIEDEL, J.: The distribution of electrons round impurities in monovalent metals. Philosophical Magazine (1952), vol. 43(337):p. 153

[Fro50] Froehlich, H.: Theory of the Superconducting State. I. The Ground State at the Absolute Zero of Temperature. Physical Review (1950), vol. 79(5):pp. 845-856, URL http://link.aps.org/doi/10.1103/PhysRev.79.845

[Geg08] Gegenwart, Philipp; Si, Qimiao and Steglich, Frank: Quantum criticality in heavy-fermion metals. Nature Physics (2008), vol. 4(3):pp. 
186-197, URL http://han.sub.uni-goettingen.de/han/60156/www . nature com/nphys/journal/v4/n3/full/nphys892.html

[Geo96] Georges, Antoine; Krauth, Werner and Rozenberg, Marcelo J.: Dynamical mean-field theory of strongly correlated fermion systems and the limit of infinite dimensions. Reviews of Modern Physics (1996), vol. 68(1):pp. 13-125, URL http://rmp.aps.org/abstract/RMP/v68/i1/p13_1

[GM54] Gell-Mann, M. and Low, F.: Quantum Electrodynamics at Small Distances. Physical Review (1954), vol. 95(5):pp. 1300-1312, URL http://link.aps.org/ doi/10.1103/PhysRev.95.1300

[Gre83] GREwe, N.: Spectral properties of the Anderson model. Zeitschrift für Physik B Condensed Matter (1983), vol. 53(4):pp. 271-282, URL http://link.springer com/10.1007/BF01436289

[Gre84] Grewe, N.: Heavy fermion superconductivity. Zeitschrift für Physik B Condensed Matter (1984), vol. 56(2):pp. 111-118, URL http://link.springer.com/10. 1007/BF01469691

[Gre91] Grewe, N. and Steglich, F.: Heavy Fermions. Handbook of Physics and Chemistry of Rare Earths (1991), vol. 14:p. 343

[Gre06] Grenzebach, Claas; Anders, Frithjof; Czycholl, Gerd and Pruschke, Thomas: Transport properties of heavy-fermion systems. Physical Review $B$ (2006), vol. 74(19):p. 195119, URL http://link.aps.org/doi/10.1103/ PhysRevB.74.195119

[Gus00] Gusmao, M. A. and Aligia, A. A.: Superconductivity in the Kondo lattice: a mean-field approach (2000), URL http://arxiv.org/abs/cond-mat/0011383

[Hew93a] Hewson, A.: Renormalized perturbation expansions and Fermi liquid theory. Physical Review Letters (1993), vol. 70(25):pp. 4007-4010, URL http://han.sub.uni-goettingen.de/han/52535_0/prl.aps.org/ abstract/PRL/v70/i25/p4007_1

[Hew93b] Hewson, Alex C.: The Kondo problem to heavy fermions, Cambridge University Press (1993)

[Hol59] Holstein, T: Studies of polaron motion. Annals of Physics (1959), vol. 8(3):pp. 325-342, URL http://dx.doi.org/10.1016/0003-4916(59)90002-8

[Hos10] Hoshino, Shintaro; Otsuki, Junya and Kuramoto, Yoshio: Itinerant antiferromagnetism in infinite dimensional Kondo lattice. Physical Review B (2010), vol. 81(11):p. 113108, URL http://link.aps.org/doi/10.1103/PhysRevB.81. 113108

[Hos13] Hoshino, Shintaro and Kuramoto, Yoshio: Itinerant vs Localized HeavyElectron Magnetism (2013):p. 5, URL http://arxiv.org/abs/1304.4325

[How12] Howczak, Olga; Kaczmarczyk, Jan and Spalek, J: From magnetic and Kondocompensated states to unconventional superconductivity in heavy fermions: a unified approach. arXiv preprint arXiv:1209.0621 (2012):p. 5, URL http: //arxiv.org/abs/1209.0621

[Hub64] HubBaRD, J.: Electron Correlations in Narrow Energy Bands. III. An Improved Solution. Proceedings of the Royal Society A: Mathematical, Physi- 
cal and Engineering Sciences (1964), vol. 281(1386):pp. 401-419, URL http: //rspa.royalsocietypublishing.org/cgi/doi/10.1098/rspa.1964.0190

[Kad66] KadAnoff, Leo P.: Scaling laws for Ising model near \$T_c\$. Physics (1966), vol. 2:p. 263

[Kas56] KasuyA, Tadao: A Theory of Metallic Ferro- and Antiferromagnetism on Zener's Model. Progress of Theoretical Physics (1956), vol. 16(1):pp. 45-57, URL http: //ptp.oxfordjournals.org/content/16/1/45.abstract

[Km80a] KrishnA-MURThY, H.; Wilkins, J. and Wilson, K.: Renormalization-group approach to the Anderson model of dilute magnetic alloys. I. Static properties for the symmetric case. Physical Review B (1980), vol. 21(3):pp. 1003-1043, URL http://link .aps .org/doi/10.1103/PhysRevB.21.1003

[Km80b] Krishna-murthy, H.; Wilkins, J. and Wilson, K.: Renormalization-group approach to the Anderson model of dilute magnetic alloys. II. Static properties for the asymmetric case. Physical Review B (1980), vol. 21(3):pp. 1044-1083, URL http://link.aps.org/doi/10.1103/PhysRevB.21.1044

[Kon64] Kondo, Jun: Resistance Minimum in Dilute Magnetic Alloys. Progress of Theoretical Physics (1964), vol. 32(1):pp. 37-49, URL http://ptp.ipap.jp/link? PTP/32/37/

[Kur83] Kuramoto, Y.: Self-consistent perturbation theory for dynamics of valence fluctuations. Zeitschrift für Physik B Condensed Matter (1983), vol. 53(1):pp. 37-52, URL http://link. springer.com/10.1007/BF01578246

[Lac79] Lacroix, C. and Cyrot, M.: Phase diagram of the Kondo lattice. Physical Review B (1979), vol. 20(5):pp. 1969-1976, URL http://link.aps.org/doi/ 10.1103/PhysRevB.20.1969

[Lan66] LANGRETH, David: Friedel Sum Rule for Anderson's Model of Localized Impurity States. Physical Review (1966), vol. 150(2):pp. 516-518, URL http://link. aps.org/doi/10.1103/PhysRev.150.516

[Lee06] LeE, Patrick A. and Wen, Xiao-Gang: Doping a Mott insulator: Physics of hightemperature superconductivity. Reviews of Modern Physics (2006), vol. 78(1):pp. 17-85, URL http://link.aps.org/doi/10.1103/RevModPhys.78.17

[Loe07] Loehneysen, Hilbert v. and Woelfle, Peter: Fermi-liquid instabilities at magnetic quantum phase transitions. Reviews of Modern Physics (2007), vol. 79(3):pp. 1015-1075, URL http://arxiv.org/abs/cond-mat/0606317

[Lut60] Luttinger, J. and WARD, J.: Ground-State Energy of a Many-Fermion System. II. Physical Review (1960), vol. 118(5):pp. 1417-1427, URL http://link.aps org/doi/10.1103/PhysRev.118.1417

[Mah00] Mahan, Gerald D.: Many-Particle Physics, Kluwer Academic Publishers Group, 3 edn. (2000)

[Mai05] Maier, Thomas; Jarrell, Mark; Pruschke, Thomas and Hettler, Matthias: Quantum cluster theories. Reviews of Modern Physics (2005), vol. 77(3):pp. 1027-1080, URL http://link.aps.org/doi/10.1103/RevModPhys.77.1027

[Mar82] Martin, Richard: Fermi-Surfae Sum Rule and its Consequences for Periodic Kondo and Mixed-Valence Systems. Physical Review Letters (1982), vol. 48(5):pp. 
362-365, URL http://link.aps.org/doi/10.1103/PhysRevLett.48.362

[Mas13] Masuda, Keisuke and Yamamoto, Daisuke: Formation of Cooper pairs between conduction and localized electrons in heavy-fermion superconductors. Physical Review B (2013), vol. 87(1):p. 014516, URL http://link.aps.org/doi/10. 1103/PhysRevB.87.014516

[Mei30a] MeIssner, W. and Voigt, B.: Messungen mit Hilfe von flüssigem Helium XI Widerstand der reinen Metalle in tiefen Temperaturen. Annalen der Physik (1930), vol. 399(8):pp. 892-936, URL http://doi.wiley.com/10.1002/andp. 19303990803

[Mei30b] Meissner, W. and Voigt, B.: Messungen mit Hilfe von flüssigem Helium XI Widerstand der reinen Metalle in tiefen Temperaturen. Annalen der Physik (1930), vol. 399(7):pp. 761-797, URL http://doi.wiley.com/10.1002/andp. 19303990702

[Met89] Metzner, Walter and Vollhardt, Dieter: Correlated Lattice Fermions in infinite Dimensions. Physical Review Letters (1989), vol. 62(3):pp. 324-327, URL http://link . aps .org/doi/10.1103/PhysRevLett.62.324

[MH89] MülleR-HaRTMann, E.: Correlated fermions on a lattice in high dimensions. Zeitschrift für Physik B Condensed Matter (1989), vol. 74(4):pp. 507-512, URL http://link.springer.com/10.1007/BF01311397

[Miy86] Miyake, K.; Schmitt-Rink, S. and Varma, C.: Spin-fluctuation-mediated even-parity pairing in heavy-fermion superconductors. Physical Review B (1986), vol. 34(9):pp. 6554-6556, URL http://link.aps.org/doi/10.1103/PhysRevB. 34.6554

[Miz11] Mizukami, Y.; Shishido, H.; Shibauchi, T.; Shimozawa, M.; Yasumoto, S.; Watanabe, D.; Yamashita, M.; Ikeda, H.; Terashima, T.; Kontani, H. and MatsudA, Y.: Extremely strong-coupling superconductivity in artificial two-dimensional Kondo lattices. Nature Physics (2011), vol. 7(11):pp. 849-853, URL http://dx.doi .org/10.1038/nphys2112

[Nam60] NambU, Yoichiro: Quasi-Particles and Gauge Invariance in the Theory of Superconductivity. Physical Review (1960), vol. 117(3):pp. 648-663, URL http://link.aps.org/doi/10.1103/PhysRev.117.648

[Nor87] NORMAN, M.: Mass renormalizations and superconductivity in heavyfermion UPt_\{3\}. Physical Review Letters (1987), vol. 59(2):pp. 232235, URL http://han.sub.uni-goettingen.de/han/52538_0/prl.aps.org/ abstract/PRL/v59/i2/p232_1

[Nor88] Norman, M.: Solutions of the magnetic Eliashberg equations for heavy-fermion superconductors. Physical Review B (1988), vol. 37(10):pp. 4987-4995, URL http://link.aps.org/doi/10.1103/PhysRevB.37.4987

[Nor03] Norman, M.; Si, Qimiao; Bazaliy, Ya. and Ramazashvili, R.: Hall Effect in Nested Antiferromagnets near the Quantum Critical Point. Physical Review Letters (2003), vol. 90(11):p. 116601, URL http://link.aps.org/doi/10.1103/ PhysRevLett.90.116601

[Nou09] Nourafkan, Reza and NAFARI, Nasser: Phase diagram of the Holstein- 
Kondo lattice model at half filling. Physical Review B (2009), vol. 79(7):p. 075122, URL http://han.sub.uni-goettingen.de/han/52538_0/prb.aps org/abstract/PRB/v79/i7/e075122

[Noz74] NozIERES, P.: A fermi-liquid description of the Kondo problem at low temperatures. Journal of Low Temperature Physics (1974), vol. 17(1-2):pp. 31-42, URL http://link.springer.com/10.1007/BF00654541

[Ohk84] OHKaWA, Fusayoshi J. and FukuYama, Hidetoshi: Anisotropic Superconductivity in the Kondo Lattice. Journal of the Physics Society Japan (1984), vol. 53(12):pp. 4344-4352, URL http://jpsj .ipap.jp/link?JPSJ/53/4344/

[Oka09] Okane, T.; Ohkochi, T.; Takeda, Y.; Fujimori, S.-i.; Yasui, A.; Saitoh, Y.; Yamagami, H.; Fujimori, A.; Matsumoto, Y.; Sugi, M.; Kimura, N.; Komatsubara, T. and Aoki, H.: 4f-Derived Fermi Surfaces of CeRu2(Si1xGex)2 near the Quantum Critical Point: Resonant Soft-X-Ray ARPES Study. Physical Review Letters (2009), vol. 102(21):p. 216401, URL http://link.aps org/doi/10.1103/PhysRevLett.102.216401

[Onu87] Onuki, Y. and Komatsubara, T.: Heavy fermion state in CeCu6. Journal of Magnetism and Magnetic Materials (1987), vol. 63-64(null):pp. 281-288, URL http://dx.doi.org/10.1016/0304-8853(87)90587-7

[Onu13] Onuki, Yoshichika; Settai, Rikio; Miura, Yasunao; Tsutsumi, Hiroki; Honda, Fuminori and Harima, Hisatomo: Heavy-fermion superconductivity and Fermisurface properties under pressure. physica status solidi (b) (2013), vol. 250(3):pp. 583-588, URL http://doi.wiley.com/10.1002/pssb.201200913

[Osh00] OshikawA, Masaki: Topological Approach to Luttinger's Theorem and the Fermi Surface of a Kondo Lattice. Physical Review Letters (2000), vol. 84(15):pp. 3370-3373, URL http://link.aps.org/doi/10.1103/PhysRevLett.84.3370

[Ots09] Otsuki, Junya; Kusunose, Hiroaki and Kuramoto, Yoshio: Evolution of a Large Fermi Surface in the Kondo Lattice. Physical Review Letters (2009), vol. 102(1), URL http://prl.aps.org/abstract/PRL/v102/i1/e017202

[Par11] Park, Tuson; Sidorov, V A; Lee, H; Ronning, F; Bauer, E D; SarraO, J L and Thompson, J D: Unconventional quantum criticality in the pressure-induced heavy-fermion superconductor CeRhIn(5). Journal of physics. Condensed matter : an Institute of Physics journal (2011), vol. 23(9):p. 094218, URL http: //www.ncbi.nlm.nih.gov/pubmed/21339571

[Pas04] Paschen, S; Lühmann, T; Wirth, S; Gegenwart, P; Trovarelli, O; Geibel, C; Steglich, F; Coleman, P and Si, Q: Hall-effect evolution across a heavyfermion quantum critical point. Nature (2004), vol. 432(7019):pp. 881-5, URL http://dx.doi.org/10.1038/nature03129

[Pat10] Patil, Swapnil; Pandey, Sudhir K; Medicherla, V R R; Singh, R S; Bindu, R; SAmpathkumaran, E V and Maiti, Kalobaran: Importance of conduction electron correlation in a Kondo lattice. Journal of physics. Condensed matter : an Institute of Physics journal (2010), vol. 22(25):p. 255602, URL http: //stacks. iop.org/0953-8984/22/i=25/a=255602

[Per07] Perkins, N.; Núñez Regueiro, M.; Coqblin, B. and Iglesias, J.: Under- 
screened Kondo lattice model applied to heavy fermion uranium compounds. Physical Review B (2007), vol. 76(12):p. 125101, URL http://link.aps.org/ doi/10.1103/PhysRevB.76.125101

[Pet06] Peters, Robert; Pruschke, Thomas and Anders, Frithjof: Numerical renormalization group approach to Greens functions for quantum impurity models. Physical Review B (2006), vol. 74(24):p. 245114, URL http://link.aps.org/ doi/10.1103/PhysRevB.74.245114

[Pet07] Peters, Robert and Pruschke, Thomas: Magnetic phases in the correlated Kondo-lattice model. Physical Review B (2007), vol. 76(24), URL http://prb aps.org/abstract/PRB/v76/i24/e245101

[Pet09] Peters, Robert: Magnetic Phases in the Hubbard Model, Ph.D. thesis, University of Göttingen (2009)

[Pet12] Peters, Robert; Kawakami, Norio and Pruschke, Thomas: Spin-Selective Kondo Insulator: Cooperation of Ferromagnetism and the Kondo Effect. Physical Review Letters (2012), vol. 108(8), URL http://prl.aps.org/abstract/PRL/ $\mathrm{v} 108 / \mathrm{i} / \mathrm{e} 086402$

[Pla06] Plakida, N M: Thermodynamic Green functions in theory of superconductivity. Condesed Matter Physics (2006), vol. 9(3(47)):pp. 619-633, URL http://www icmp.lviv.ua/journal/zbirnyk.47/015/art15.pdf

[Pru00] Pruschke, Th; Bulla, R and Jarrell, M: The low-energy scale of the periodic Anderson model. Physical Review B (2000), vol. 61(19):p. 12799, URL http: //arxiv.org/abs/cond-mat/0001357

[Rac10] Raczkowski, M.; Zhang, P.; Assaad, F. F.; Pruschke, T. and Jarrell, M.: Phonons and the coherence scale of models of heavy fermions. Physical Review B (2010), vol. 81(5):p. 054444, URL http://link.aps.org/doi/10. 1103/PhysRevB.81.054444

[Raz84] Razafimandimby, H.; Fulde, P. and Keller, J.: On the theory of superconductivity in Kondo lattice systems. Zeitschrift für Physik B Condensed Matter (1984), vol. 54(2):pp. 111-120, URL http://link.springer.com/10. $1007 / \mathrm{BF} 01388062$

[Rea83] Read, N and Newns, D M: On the solution of the Coqblin-Schreiffer Hamiltonian by the large-N expansion technique. Journal of Physics C: Solid State Physics (1983), vol. 16(17):pp. 3273-3295, URL/han/33361/iopscience.iop.org/ 0022-3719/16/17/014

[Ric85] RIce, T. and UedA, K.: Gutzwiller Variational Approximation to the HeavyFermion Ground State of the Periodic Anderson Model. Physical Review Letters (1985), vol. 55(9):pp. 995-998, URL http://han.sub.uni-goettingen.de/ han/52538_0/prl.aps.org/abstract/PRL/v55/i9/p995_1

[Rub08] Rubtsov, A.; Katsnelson, M. and Lichtenstein, A.: Dual fermion approach to nonlocal correlations in the Hubbard model. Physical Review B (2008), vol. 77(3):p. 033101, URL http://link.aps.org/doi/10.1103/PhysRevB.77. 033101

[Rud54] Ruderman, M. A. and Kittel, C.: Indirect Exchange Coupling of Nuclear Mag- 
netic Moments by Conduction Electrons. Physical Review (1954), vol. 96(1):pp. 99-102, URL http://link.aps.org/doi/10.1103/PhysRev.96.99

[Só5] SÉnéchal, David; Lavertu, P.-L.; Marois, M.-A. and Tremblay, A.-M.: Competition between Antiferromagnetism and Superconductivity in High-Tc Cuprates. Physical Review Letters (2005), vol. 94(15):p. 156404, URL http: //link.aps.org/doi/10.1103/PhysRevLett.94.156404

[Sac12] Sachdev, Subir; Metlitski, Max A and Punk, Matthias: Antiferromagnetism in metals: from the cuprate superconductors to the heavy fermion materials. Journal of physics. Condensed matter : an Institute of Physics journal (2012), vol. 24(29):p. 294205, URL/han/491_2/iopscience.iop.org/0953-8984/24/ 29/294205/article/

[Sak93] SAKai, Osamu; Shimizu, Yukihiro; Shiba, Hiroyuki and SATori, Koji: Numerical Renormalization Group Study of Magnetic Impurities in Superconductors. II. Dynamical Excitation Spectra and Spatial Variation of the Order Parameter. Journal of the Physics Society Japan (1993), vol. 62(9):pp. 3181-3197, URL http://jpsj.ipap.jp/link?JPSJ/62/3181/

[San02] Santos, C. and Nolting, W.: Ferromagnetism in the Kondo-lattice model. Physical Review B (2002), vol. 65(14):p. 144419, URL http://link.aps.org/ doi/10.1103/PhysRevB.65.144419

[Sat92] SATori, Koji; ShibA, Hiroyuki; SAKaI, Osamu and Shimizu, Yukihiro: Numerical Renormalization Group Study of Magnetic Impurities in Superconductors. Journal of the Physical Society of Japan (1992), vol. 61(9):pp. 3239-3254, URL http://jpsj .ipap.jp/link? JPSJ/61/3239/

[Sca66] Scalapino, D.; Schrieffer, J. and Wilkins, J.: Strong-Coupling Superconductivity. I. Physical Review (1966), vol. 148(1):pp. 263-279, URL http: //link.aps.org/doi/10.1103/PhysRev.148.263

[Sca86] ScAlapino, D.; LoH, E. and Hirsch, J.: d-wave pairing near a spin-densitywave instability. Physical Review B (1986), vol. 34(11):pp. 8190-8192, URL http://link.aps.org/doi/10.1103/PhysRevB.34.8190

[Sch66] Schrieffer, J. and WolfF, P.: Relation between the Anderson and Kondo Hamiltonians. Physical Review (1966), vol. 149(2):pp. 491-492, URL http: //link.aps.org/doi/10.1103/PhysRev.149.491

[Sia12] Siahatgar, M; Schmidt, B; Zwicknagl, G and Thalmeier, P: Moment screening in the correlated Kondo lattice model. New Journal of Physics (2012), vol. 14(10):p. 103005, URL http://stacks.iop.org/1367-2630/14/i=10/a= 103005

[Sin02] Sinjukow, P. and Nolting, W.: Exact mapping of periodic Anderson model to Kondo lattice model. Physical Review B (2002), vol. 65(21):p. 212303, URL http://link.aps.org/doi/10.1103/PhysRevB.65.212303

[Ste79] Steglich, F.; Aarts, J.; Bredl, C.; Lieke, W.; Meschede, D.; Franz, W. and Schäfer, H.: Superconductivity in the Presence of Strong Pauli Paramagnetism: CeCu_\{2\}Si_\{2\}. Physical Review Letters (1979), vol. 43(25):pp. 1892-1896, URL http://link.aps.org/doi/10.1103/PhysRevLett.43.1892 
[Ste84] Stewart, G R: Heavy-fermion systems. Reviews of Modern Physics (1984), vol. 56(4):pp. 755-787, URL http://link.aps.org/doi/10.1103/RevModPhys 56.755

[Ste01] Stewart, G.: Non-Fermi-liquid behavior in d- and f-electron metals. Reviews of Modern Physics (2001), vol. 73(4):pp. 797-855, URL http://link.aps.org/ doi/10.1103/RevModPhys.73.797

[Ste12a] STEGLICH, F: Heavy-fermion superconductivity in the Kondo-lattice system CeCu 2 Si 2. Journal of Physics: Conference Series (2012), vol. 400(2):p. 022111, URL http://stacks.iop.org/1742-6596/400/i=2/a=022111

[Ste12b] Steglich, F; Arndt, J; Stockert, O; Friedemann, S; Brando, M; Klingner, C; Krellner, C; Geibel, C; Wirth, S; Kirchner, S and SI, Q: Magnetism, f-electron localization and superconductivity in 122-type heavy-fermion metals. Journal of physics. Condensed matter : an Institute of Physics journal (2012), vol. 24(29):p. 294201, URL http://stacks.iop.org/ $0953-8984 / 24 / i=29 / a=294201$

[Sto11] Stockert, O.; Arndt, J.; Faulhaber, E.; Geibel, C.; Jeevan, H. S.; KirchNer, S.; Loewenhaupt, M.; Schmalzl, K.; Schmidt, W.; Si, Q. and Steglich, F.: Magnetically driven superconductivity in CeCu2Si2. Nature Physics (2011), vol. 7(2):pp. 119-124, URL http://dx.doi.org/10.1038/ nphys 1852

[Sto12] Stockert, Oliver; KIrchner, Stefan; Steglich, Frank and Si, Qimiao: Superconductivity in Ce- and U-Based 122 Heavy-Fermion Compounds. Journal of the Physical Society of Japan (2012), vol. 81(1):p. 011001, URL http: //jpsj.ipap.jp/link?JPSJ/81/011001/

[Stu53] Stueckelberg, Ernst Carl Gerlach and Petermann, Andre: La normalization des constantes dans la theorie des quanta. Helvetica physica acta (1953), vol. 26:p. 499

[Tac84] Tachiki, M. and MaEkaWA, S.: Superconductivity in the Kondo lattice. Physical Review B (1984), vol. 29(5):pp. 2497-2502, URL http://han.sub.uni-goettingen.de/han/52538_0/prb.aps.org/ abstract/PRB/v29/i5/p2497_1

[Tho12] Thomas, Christopher; Simoes, Acirete S da Rosa; Iglesias, J R; Lacroix, C and Coqublin, B: Application of the underscreened Kondo lattice model to neptunium compounds. Journal of Physics: Conference Series (2012), vol. 391:p. 012174, URL http://stacks.iop.org/1742-6596/391/i=1/a=012174?key= crossref.88de508953618d1ccdef $36 \mathrm{fbb7e} 47817$

[TZ98] Tahvildar-Zadeh, A. N.; Hettler, M. H. and Jarrell, M.: Superconducting instability in the periodic Anderson model. Philosophical Magazine Part B (1998), vol. 78(4):pp. 365-374, URL http://www.tandfonline.com/doi/abs/ $10.1080 / 13642819808206733$

[Voj10] VoutA, Matthias: Orbital-Selective Mott Transitions: Heavy Fermions and Beyond. Journal of Low Temperature Physics (2010), vol. 161(1-2):pp. 203-232, URL http://link.springer.com/10.1007/s10909-010-0206-3 
[Ž] ŽItKo, Rok: NRG Ljubljana, URL http://nrgljubljana.ijs.si/

[Ž07] ŽITKO, Rok: Many-particle effects in resonant tunneling of electrons through nanostructures, Phd thesis, University of Ljubljana (2007), URL http://auger ijs.si/nano/Rok_Zitko_2007_dis.pdf

[Ž09a] ŽıTKO, Rok: Convergence acceleration and stabilization of dynamical mean-field theory calculations. Physical Review B (2009), vol. 80(12):p. 125125, URL http://link.aps.org/doi/10.1103/PhysRevB.80.125125

[Žo9b] ŽıtKo, Rok and PRUschke, Thomas: Energy resolution and discretization artifacts in the numerical renormalization group. Physical Review B (2009), vol. 79(8):p. 085106, URL http://link.aps.org/doi/10.1103/PhysRevB.79.085106

[Ž11] Žıtko, Rok; Bodensiek, Oliver and PRuschke, Thomas: Effects of magnetic anisotropy on the subgap excitations induced by quantum impurities in a superconducting host. Physical Review B (2011), vol. 83(5):p. 054512, URL http://prb.aps.org/abstract/PRB/v83/i5/e054512http:// link.aps.org/doi/10.1103/PhysRevB.83.054512

[Wat07] Watanabe, Hiroshi and Ogata, Masao: Fermi-Surface Reconstruction without Breakdown of Kondo Screening at the Quantum Critical Point. Physical Review Letters (2007), vol. 99(13):p. 136401, URL http://link.aps.org/doi/ 10.1103/PhysRevLett.99.136401

[Wei07] Weichselbaum, Andreas and von Delft, Jan: Sum-Rule Conserving Spectral Functions from the Numerical Renormalization Group. Physical Review Letters (2007), vol. 99(7):p. 076402, URL http://link.aps.org/doi/10.1103/ PhysRevLett.99.076402

[Wil75] Wilson, Kenneth: The renormalization group: Critical phenomena and the Kondo problem. Reviews of Modern Physics (1975), vol. 47(4):pp. 773-840, URL http://link.aps.org/doi/10.1103/RevModPhys.47.773

[Xu 87] XU , Ji-HaI; LI, TIE-Cheng; Su, Zhao-Bing: Theory of superconductivity in a Kondo lattice. Acta Physica Sinica (1987), vol. 36:p. 613

[Yos57] Yosida, Kei: Magnetic Properties of Cu-Mn Alloys. Physical Review (1957), vol. 106(5):pp. 893-898, URL http://link.aps.org/doi/10.1103/PhysRev. 106 893

[Yos83] Yoshimori, A. and Kasai, H.: Theory of dense Kondo system. Journal of Magnetism and Magnetic Materials (1983), vol. 31-34(null):pp. 475-476, URL http://dx.doi.org/10.1016/0304-8853(83)90328-1

[Zit02] Zitzler, R.; Pruschke, Th. and Bulla, R.: Magnetism and phase separation in the ground state of the Hubbard model. The European Physical Journal B - Condensed Matter (2002), vol. 27(4):pp. 473-481, URL http://www . springerlink. com/openurl . asp?genre=article\&id=doi:10.1140/epjb/e2002-00180-3 
